\title{
Properties of hot and dense matter from relativistic heavy ion collisions
}

\author{
Peter Braun-Munzinger ${ }^{\mathrm{a}, \mathrm{b}, \mathrm{c}}$, Volker Koch ${ }^{\mathrm{d}}$, Thomas Schäfer ${ }^{\mathrm{f}}$, Johanna Stachel $^{\mathrm{e}}$ \\ ${ }^{a}$ EMMI, GSI Helmholtzzentrum fuer Schwerionenforschung, 64291 Darmstadt, Germany \\ ${ }^{b}$ Technical University, 64287 Darmstadt, Germany \\ ${ }^{c}$ FIAS, 60438 Frankfurt, Germany \\ ${ }^{d}$ Nuclear Science Division, Lawrence Berkeley National Laboratory, Berkeley, CA 94720, USA \\ ${ }^{e}$ Physikalisches Institut, Universität Heidelberg, 69120 Heidelberg, Germany \\ ${ }^{f}$ Department of Physics, North Carolina State University, Raleigh, NC 27695, USA
}

\begin{abstract}
We review the progress achieved in extracting the properties of hot and dense matter from relativistic heavy ion collisions at the relativistic heavy ion collider (RHIC) at Brookhaven National Laboratory and the large hadron collider (LHC) at CERN. We focus on bulk properties of the medium, in particular the evidence for thermalization, aspects of the equation of state, transport properties, as well as fluctuations and correlations. We also discuss the in-medium properties of hadrons with light and heavy quarks, and measurements of dileptons and quarkonia. This review is dedicated to the memory of Gerald E. Brown.
\end{abstract}

\section{Introduction}

Soon after the discovery of QCD [1], and following the realization that QCD exhibits asymptotic freedom $[2,3]$, is was recognized that QCD implies the existence of a new high temperature phase of weakly interacting quarks and gluons, termed the quark-gluon plasma $[4,5,6]$. The idea of a limiting temperature for hadronic matter predates the discovery of QCD, and a quantitative prediction $T \simeq 170 \mathrm{MeV}$ was obtained in the statistical bootstrap model of Hagedorn [7]. The existence of a new phase was confirmed in the first calculations using the lattice formulation of QCD, initially for pure $S U(2)$ gauge theory $[8,9]$.

These results inspired the community to explore the possibility to create and study the quark-gluon plasma by colliding heavy nuclei at high energy, see for example [10]. Early ideas of creating thermodynamically equilibrated matter in high energy hadronic collisions go back to Fermi [11], Landau [12], and Hagedorn [7]. The idea of colliding $U+U$ at the CERN ISR was considered, but not pursued, in the late 1960s. The subject received "subtle stimulation" [13] from a workshop on "GeV/ nucleon collisions of heavy ions" at Bear Mountain, New Preprint submitted to Elsevier

November 28, 2015 
York [14]. A meeting on ultra-relativistic heavy ion physics was convened in Berkeley in 1979 [15], which spawned a series of Quark Matter conferences that continue to this day.

An experimental relativistic heavy ion program began at the Bevalac facility at Lawrence Berkeley National Laboratory in the mid nineteen-seventies, initially motivated by the study of compressed nuclear matter and the search for "abnormal" states of matter, such as pion condensed matter or Lee-Wick matter $[16,17]$. These experiments discovered a number of collective phenomena [18], such as hydrodynamic flow, that are still being studied today. Exploratory experiments in the highly relativistic regime, initially carried out with rather small nuclei, began at the Brookhaven AGS and the CERN SPS accelerator in 1986. These experiments confirmed that a significant amount of energy is being deposited at mid-rapidity. It was also found that the observed particle yields are well described be the Hagedorn inspired hadron resonance model [19, 20]. There were already some surprises, such as an unexpected enhancement of low mass lepton pairs [21].

The availability of $\mathrm{Pb}$ beams at the SPS, and the beginning of the collider era at the dedicated Relativistic Heavy Ion Collider (RHIC) at Brookhaven, mark the beginning of the current era in relativistic heavy ion physics. A wealth of phenomena were discovered, many of them surprising. At CERN this includes the observation of anomalous $J / \psi$ suppression [22], the enhanced, compared to pp collisions, production of strange hadrons [23], as well as a low-mass enhancement coupled with the disappearance of the rho-peak in dilepton measurements [24].

The central discoveries at RHIC are the observation of a large azimuthal asymmetry, known as elliptic flow $v_{2}$, in the particle yields [25], as well as a strong suppression of high energy jets and heavy quarks [26]. The observed elliptic flow was consistent with predictions from ideal hydrodynamics, which was puzzling, since one expected to find a weakly interacting quark-gluon plasma, which should not exhibit fluid dynamic behavior. Further analysis of this effect, together with the large opacity of the QGP implied by the jet quenching data, forced a paradigm shift. In particular, it was argued that, instead of the originally anticipated weakly coupled system of quarks and gluons, the experiments had discovered a strongly interacting quark-gluon plasma (sQGP) [27, 28, 29, 30].

These experimental advances were accompanied by important theoretical developments and breakthroughs. For example it was realized that by using methods developed in string theory, the holographic duality between gravitational theories in warped higher dimensional space-time and gauge theories in flat space on its boundary, one could study certain strongly interacting theories [31]. Using these techniques it was shown that theories that can be realized using holographic dualities saturate a lower bound on the shear viscosity over entropy density ratio [32]. In addition, inspired by the RHIC data, the theory community revisited 
the long-standing problem of relativistic viscous fluid dynamics and turned it into a practical tool $[33,34]$. And, last but not least, improved actions and algorithms together with increased computing power allowed lattice simulations of real QCD with realistic quark masses. These calculations for example showed that the transition at vanishing baryon density is a cross-over [35] with a pseudocritical temperature of $T \simeq 150 \mathrm{MeV}[36,37,38,39]$.

Experiments at the CERN LHC at about ten times the energy of RHIC confirmed several of the main results obtained at RHIC, such as elliptic flow and energy loss, while providing improved statistics and a much larger kinematic range for many key observables. An example is the detailed measurement of higher flow harmonics, first carried out at the LHC, which opened up a window for testing the fluctuating initial conditions with the possibility to probe the structure of nuclei at the partonic scale. LHC experiments also made a number of surprising discoveries. For example, features resembling collective flow have been observed in small systems, such as $p+P b$ and possibly even $p+p[40,41]$. Furthermore, the $\mathrm{J} / \psi$ suppression in $\mathrm{Pb}-\mathrm{Pb}$ collisions at the LHC [42] is much reduced compared to measurements at RHIC. Although this has been predicted many years before the start of the LHC [43, 44], and thus should not have been a surprise, it took the actual measurement for these ideas to be taken seriously.

While the experiments at the highest energies focus on detailed measurements of the properties of a QGP, a new program at RHIC has started with the goal to explore the QCD phase diagram at finite net baryon density. To achieve this a systematic beam energy scan down to the lowest energies available at RHIC has been carried out [45]. The first set of measurements found intriguing non-monotonic dependence on the beam energy of some of the key observables, such as proton number fluctuation and system size at freeze out as determined by Hanbury-Brown Twiss type pion correlation, as one would expect from a phase change at finite density. At the same time flow observables are remarkably insensitive to the collision energy, adding to the puzzle raised by the unexpected flow in small systems.

In this review we wish to summarize some of the recent development discussed above, and provide an introduction to recent observations and ideas. We wish to dedicate this review to the memory of Gerry Brown, our teacher, mentor, and friend. After many years of working in nuclear structure and nuclear astrophysics, Gerry developed an interest in relativistic heavy ion collisions as a way of pinning down the nuclear equation of state at densities above nuclear saturation density, which is of interest for type II supernova explosions [46, 47]. Gerry was well aware of the theoretical and experimental developments in the field. He had been a speaker at the Bear Mountain workshop, describing his work on pion condensation in nuclear matter [14]. He applied his expertise in the theory of collective modes to the problem of hadrons in hot and dense matter [48], and, in 
collaboration with M. Rho, developed the idea of Brown-Rho scaling [49], which drove much of the interest in dilepton experiments for many years. In general, his interests focused on bulk phenomena, like the equation of state [50], and the properties and spectra of hadrons [51]. Gerry followed the early RHIC results, as well as improved results from CERN, with great interest, but he fell seriously ill before the start of the LHC. He certainly would have been excited to see the first results. We spent many hours discussing the physics of relativistic heavy ions with him in the office, at lunch in the nuclear theory common room, or at dinner in his Setauket home.

This review focuses on issues that were closest to his interests, the bulk properties of hot and dense matter, the spectra of produced particles and the evidence for thermalization, as well as the in-medium properties of hadrons. The review is organized as follows. In Section 2 we provide an overview of the phase diagram of QCD, and discuss the equation of state. In Section 3 we summarize experimental results on hadron spectra, as well as the evidence that thermalization is achieved in relativistic heavy ion collisions. The theory of locally equilibrated matter, relativistic fluid dynamics, is discussed in Section 4, together with ongoing efforts to determine transport properties of the QGP, and discover the limits of applicability of fluid dynamics. In Section 5 we consider fluctuations and correlations, as well as their role is the extraction of freeze-out properties and the search for the critical point. We close with Sections devoted to the in-medium properties of hadrons, Section 6, the production of dileptons, Section 7, and the spectra of hadrons containing heavy quarks, Section 8. Finally, we provide a brief outlook in Section 9.

\section{The phase structure of QCD}

\subsection{The phases of $Q C D$}

\subsubsection{The $Q C D$ Vacuum}

Strongly interacting matter has a rich phase structure, which includes a nuclear liquid phase, a hadronic gas, and the quark-gluon plasma. All these states of matter are described by quantum chromodynamics (QCD), which is the theory of quarks and gluons and their interactions. The complicated phenomenology of the strong interaction is encoded in a deceptively simple Lagrangian. The Lagrangian is formulated in terms of quark fields $q_{\alpha f}^{c}$ and gluon fields $A_{\mu}^{a}$. Here, $\alpha=1, \ldots, 4$ is a Dirac spinor index, $c=1, \ldots, N_{c}$ with $N_{c}=3$ is a color index, and $f=u p$, down, strange, charm, bottom, top is a flavor index.

The dynamics of the theory is governed by the color degrees of freedom. The gluon field $A_{\mu}^{a}$ is a vector field labeled by an adjoint color index $a=1, \ldots, 8$. The octet of gluon fields can be used to construct a matrix valued field $A_{\mu}=A_{\mu}^{a} \frac{\lambda^{a}}{2}$, 
where $\lambda^{a}$ is a set of traceless, Hermitian, $3 \times 3$ matrices. The QCD Lagrangian is

$$
\mathcal{L}=-\frac{1}{4} G_{\mu \nu}^{a} G_{\mu \nu}^{a}+\sum_{f}^{N_{f}} \bar{q}_{f}\left(i \gamma^{\mu} D_{\mu}-m_{f}\right) q_{f},
$$

where $G_{\mu \nu}^{a}$ is the QCD field strength tensor defined by

$$
G_{\mu \nu}^{a}=\partial_{\mu} A_{\nu}^{a}-\partial_{\nu} A_{\mu}^{a}+g f^{a b c} A_{\mu}^{b} A_{\nu}^{c},
$$

and $f^{a b c}=4 i \operatorname{Tr}\left(\left[\lambda^{a}, \lambda^{b}\right] \lambda^{c}\right)$ is a set of numbers called the $S U(3)$ structure constants. The covariant derivative acting on the quark fields is

$$
i D_{\mu} q=\left(i \partial_{\mu}+g A_{\mu}^{a} \frac{\lambda^{a}}{2}\right) q
$$

and $m_{f}$ is the mass of the quarks. The terms in Eq. (1) describe the interaction between quarks and gluons, as well as nonlinear three and four-gluon interactions. We observe that, except for the number of flavors and their masses, the structure of the QCD Lagrangian is completely fixed by the local $S U(3)$ color symmetry.

For the purpose of understanding hadronic matter and the quark-gluon plasma we can consider the light flavors (up, down, and strange) to be approximately massless, and the heavy flavors (charm, bottom, top) to be infinitely massive. In this limit the QCD Lagrangian contains a single dimensionless parameter, the coupling constant $g$. If quantum effects are taken into account the coupling becomes scale dependent $[2,3]$. At leading order the running coupling constant is

$$
g^{2}\left(q^{2}\right)=\frac{16 \pi^{2}}{b_{0} \log \left(q^{2} / \Lambda_{Q C D}^{2}\right)}, \quad b_{0}=\frac{11}{3} N_{c}-\frac{2}{3} N_{f}
$$

where $q$ is a characteristic momentum and $N_{f}$ is the number of active flavors $\left(N_{f}=3\right.$ in the approximation considered here). The running coupling implies that, as a quantum theory, QCD is not characterized by a dimensionless coupling but by a dimensionful scale, the QCD scale parameter $\Lambda_{Q C D}$. This effect is known as dimensional transmutation [52]. We also observe that the coupling decreases with increasing momentum. This is the phenomenon of asymptotic freedom $[2$, 3]. The flip side of asymptotic freedom is anti-screening, or confinement: The effective interaction between quarks increases with distance.

In massless QCD the scale parameter is an arbitrary parameter (a QCD "standard kilogram"), and all observables are dimensionless ratios like $m_{p} / \Lambda_{Q C D}$, where $m_{p}$ is the mass of the proton. If QCD is embedded into the electroweak sector of the standard model, and quarks acquire masses by electroweak symmetry breaking, then the QCD scale is fixed by the choice of units in the standard 


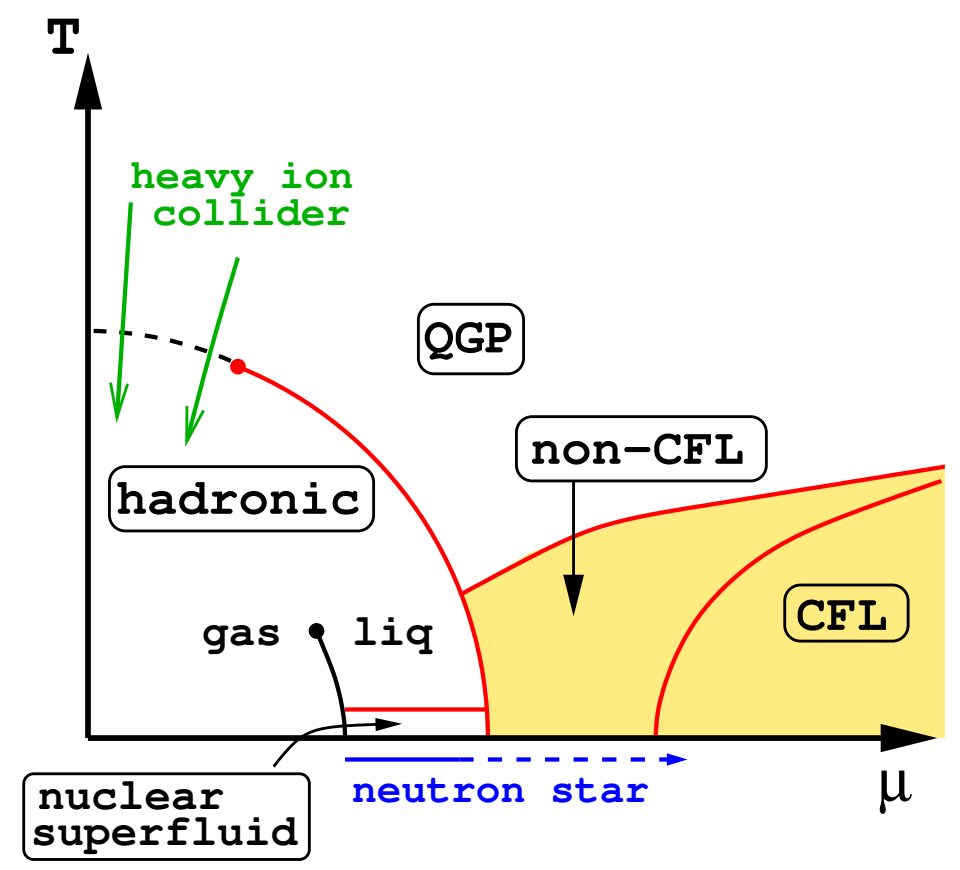

Figure 1: Schematic phase diagram of QCD as a function of temperature $T$ and baryon chemical potential $\mu$. QGP refers to the quark-gluon plasma. The CFL (color-flavor locked) phase is the color superconducting phase that occurs at asymptotically large chemical potential. The red and black points denote the critical endpoints of the chiral and nuclear liquid-gas phase transitions, respectively. The dashed line is the chiral pseudo-critical line associated with the crossover transition at low temperature. The green arrows denote the regions of the phase diagram that are being explored by the experimental heavy ion programs at the LHC and RHIC.

model. A number that is commonly quoted is the value of the QCD fine structure constant $\alpha_{s}=g^{2} /(4 \pi)$ at the $Z$ boson pole, $\alpha_{s}\left(m_{z}\right)=0.1184 \pm 0.0007$ [53]. The numerical value of $\Lambda_{Q C D}$ depends on the renormalization scheme used to derive Eq. (4). Physical masses, as well as the value of $b_{0}$, are independent of this choice. In the modified minimal subtraction $(\overline{M S})$ scheme one finds $\Lambda_{Q C D} \simeq 200 \mathrm{MeV}$ [53].

Asymptotic freedom and the symmetries of QCD determine the basic phases of strongly interacting matter that appear in the QCD phase diagram shown in Fig. 1. In this figure we show the phases of QCD as a function of the temperature $T$ and the baryon chemical potential $\mu$. The chemical potential $\mu$ controls the baryon density $\rho$, defined as $1 / 3$ times the number density of quarks minus the number density of anti-quarks.

At zero temperature and chemical potential the interaction between quarks is dominated by large distances and the effective coupling is large. As a con- 
sequence, quarks and gluons are permanently confined in color singlet hadrons, with masses of order $\Lambda_{Q C D}$. For example, the proton has a mass of $m_{p}=935$ $\mathrm{MeV}$. If we view the proton as composed of three constituent quarks this implies that quarks have effective masses $m_{Q} \simeq m_{P} / 3 \simeq \Lambda_{Q C D}$. This should be compared to the bare up and down quark masses which are of the order $10 \mathrm{MeV}$.

Strong interactions between quarks, anti-quarks, and gluons lead to the formation of vacuum condensates of color-singlet bosonic states. In particular, the QCD ground state supports a condensate of $\bar{q} q$ pairs, $\langle\bar{q} q\rangle \simeq-\Lambda_{Q C D}^{3}[54,55,56]$. The quark condensate couples left and right handed fermions, $\bar{q} q=\bar{q}_{L} q_{R}+\bar{q}_{R} q_{R}$, and it is diagonal in flavor space, $\left\langle\bar{q}_{f} q_{g}\right\rangle=\delta_{f g}\langle\bar{q} q\rangle$. Quark-anti-quark condensation spontaneously breaks the approximate chiral $S U(3)_{L} \times S U(3)_{R}$ flavor symmetry of the QCD Lagrangian down to its vectorial subgroup, the flavor symmetry $S U(3)_{V}$. Chiral symmetry breaking implies the existence of Goldstone bosons, massless modes with the quantum numbers of the generators of the broken axial symmetry $S U(3)_{A}$. These particles are pions, kaons, and etas. The $S U(3)_{L} \times S U(3)_{R}$ is explicitly broken by quark masses, and the mass of the charged pion is $m_{\pi}=139 \mathrm{MeV}$, which is not much smaller than $\Lambda_{Q C D}$. The lightest non-Goldstone particle is the rho meson, which has a mass $m_{\rho}=770$ $\mathrm{MeV}$.

\subsubsection{High temperature $Q C D$}

At very high temperature quarks and gluons have thermal momenta $p \sim T \gg$ $\Lambda_{Q C D}$. Asymptotic freedom implies that these particles are weakly interacting, and that they form a plasma of mobile color charges, the quark-gluon plasma [6, 57]. We note that the argument that the QGP at asymptotically high temperature is weakly coupled is somewhat more subtle than it might appear at first sight. If two particles in the plasma interact via large angle scattering then the momentum transfer is large, and the effective coupling is weak because of asymptotic freedom. However, the color Coulomb interaction is dominated by small angle scattering, and it is not immediately clear why the effective interaction that governs small angle scattering is weak. The important point is that in a high temperature plasma there is a large thermal population $\left(n \sim T^{3}\right)$ of mobile charges that screen the interaction at distances beyond the Debye length $r_{D} \sim 1 /(g T)$. We also note that even in the limit $T \gg \Lambda_{Q C D}$ the QGP contains a non-perturbative sector of static magnetic color fields [58]. This sector is strongly coupled, but it does not contribute to thermodynamic or transport properties of the plasma in the limit $T \rightarrow \infty$, see the discussion in Section 6.3.

The plasma phase exhibits neither color confinement nor chiral symmetry breaking. This means that the high temperature QGP phase must be separated from the low temperature hadronic phase by a phase transition. The nature of this transition is very sensitive to the values of the quark masses. In QCD 
with massless $u, d$ and infinitely massive $s, c, b, t$ quarks the transition is second order [59]. In the case of massless (or sufficiently light) $u, d, s$ quarks the transition is first order. Lattice simulations show that for realistic quark masses, $m_{u} \simeq m_{d} \simeq$ $10 \mathrm{MeV}$ and $m_{s} \simeq 120 \mathrm{MeV}$, the phase transition is a rapid crossover [35, 38]. A pseudo-critical transition temperature can be defined by locating the maximum of the chiral susceptibility, that means by identifying the point at which fluctuations of the chiral order parameter are largest. The result is $T_{c} \simeq 151 \pm 3 \pm 3 \mathrm{MeV}$ [36, 37], consistent with the determination $154 \pm 9 \mathrm{MeV}$ published in [38, 39].

The transition is believed to strengthen as a function of chemical potential, so that there is a critical $\mu$ at which the crossover turns into a first order phase transition [60]. This point is the critical endpoint of the chiral phase transition. Due to the fermion sign problem it is very difficult to locate the critical endpoint using simulations on the lattice. Model calculations typically predict the existence of a critical point, but do not constrain its location. A number of exploratory lattice calculations have been performed [61, 62, 63, 64, 65, 66], but at this point it is not even clear whether the idea that the transition strengthens with increasing baryon chemical potential is correct [67]. The critical endpoint is interesting because it is the only thermodynamically stable point on the phase transition line at which the correlation length diverges (there is a similar endpoint on the nuclear liquid-gas transition line). This means that the critical point may manifest itself in heavy ion collisions in terms of enhanced fluctuations [68], see Section 5.

The $T=\mu=0$ point in the phase diagram corresponds to the vacuum state of QCD. If the chemical potential is increased at $T=0$ then initially there is no change, because at zero temperature the chemical potential $\mu$ is the energy required to add a baryon to the system, and QCD has a large mass gap for baryonic states. The first non-vacuum state one encounters along the $\mu$ axis of the phase diagram is nuclear matter, a strongly correlated superfluid composed of approximately non-relativistic neutrons and protons. Nuclear matter is selfbound, and the baryon density changes discontinuously at the onset transition, from $\rho_{B}=0$ to nuclear matter saturation density $\rho_{B}=\rho_{0} \simeq 0.15 \mathrm{fm}^{-3}$. The discontinuity decreases as nuclear matter is heated, and the nuclear-liquid gas phase transition ends in a critical point at $T \simeq 10 \mathrm{MeV}[69,70]$. Hot hadronic or nuclear matter can be described quite accurately as a weakly interacting gas of hadronic resonances, see Section 3. Empirically, the density of states for both mesons and baryons grows exponentially. This is reminiscent of the old string picture of hadronic resonances, and suggests that hadronic matter below $T_{c}$ can be viewed a Hagedorn gas.

We will show more detailed comparisons between lattice results and the hadronic resonance gas model in Section 5 . One can try to make the resonance gas model more precise by considering the limit $N_{c} \rightarrow \infty$. Witten and 't Hooft argued that in this limit hadronic resonances become narrow and weakly interacting, and 
that the $1 / N_{c}$ expansion in gauge theory can be mapped onto the perturbative expansion of a weakly coupled string theory $[71,72]$. Non-interacting relativistic strings are known to have an exponential density of states, and a limiting temperature $T_{H}$. However, $T_{H}$ cannot be precisely equal to the critical temperature of large $N_{c}$ QCD. Large $N_{c}$ QCD has a first order phase transition between a hadronic phase at low $T$ with pressure $O\left(N_{c}^{0}\right)$, and a deconfined phase at large $T$ with pressure $O\left(N_{c}^{2}\right)$. The string gas, on the other hand, has a pressure that diverges as $T \rightarrow T_{H}$ [73]. Indeed, lattice calculations in large $N_{c}$ QCD suggest that $T_{c}<T_{H}$, and that $T_{H}$ corresponds to the endpoint of a meta-stable hadronic phase above $T_{c}[74]$.

\subsubsection{High baryon density $Q C D$}

At very large chemical potential we can use arguments similar to those in the high temperature limit to establish that quarks and gluons are weakly coupled. The main difference between cold quark matter and the hot QGP is that because of the large density of states near the quark Fermi surface even weak interactions can cause qualitative changes in the ground state of dense matter. In particular, attractive interactions between quark pairs lead to color superconductivity and the formation of a $\langle q q\rangle$ condensate. Since quarks carry color, flavor, and spin labels, many superconducting phases are possible. The most symmetric of these, known as the color-flavor locked (CFL) phase, is predicted to exist at very high density $[75,76]$. In the CFL phase the diquark order parameter is $\left\langle q_{\alpha f}^{A} q_{\beta g}^{B}\right\rangle \sim \epsilon_{\alpha \beta} \epsilon^{A B C} \epsilon_{f g C}$. This order parameter has a number of interesting properties. It breaks the $U(1)$ symmetry associated with baryon number, leading to superfluidity, and it breaks the chiral $S U(3)_{L} \times S U(3)_{R}$ symmetry. Except for Goldstone modes the spectrum is fully gapped; fermions acquire a BCS-pairing gap, and gauge fields are screened by the Meissner effect. This implies that the CFL phase, even though it arises from a superdense liquid of quarks, shares many properties of superfluid nuclear matter.

The CFL phase involves equal pair-condensates $\langle u d\rangle=\langle u s\rangle=\langle d s\rangle$ of all three light quark flavors. As the density is lowered effects of the non-zero strange quark mass become more important, and less symmetric phases are likely to appear [77]. Possible phases include Bose condensates of pions and kaons, hyperon matter, states with inhomogeneous quark-anti-quark or diquark condensates, and less symmetric color superconducting phases. The intermediate $\mu$ regime in the phase diagram shown in Fig. 1 is therefore largely conjecture. We know that at low $\mu$ there is a nuclear matter phase with broken chiral symmetry and zero strangeness, and that at high $\mu$ we find the CFL phase with broken chiral symmetry but non-zero strangeness. In principle the two phases could be separated only by a continuous onset transition for strangeness [78, 79], but model calculation suggest a more complicated picture in which one or more first order transitions intervene, 
as shown in Fig. 1.

\subsection{The equation of state}

The most basic property of a phase of $\mathrm{QCD}$, and the observable that enters most directly in the theoretical description of an expanding quark-gluon plasma, is its equation of state (EOS). The EOS governs the dependence of the pressure of the system on the energy and baryon density, $P=P\left(\mathcal{E}, n_{B}\right)$, or equivalently, on the temperature and chemical potential, $P=P(T, \mu)$. Here we have used the fact that in thermodynamic equilibrium the total electric charge must be zero, and strangeness is not conserved. In a heavy ion collision the system has a net charge and strangeness is approximately conserved. However, at mid-rapidity both net isospin and strangeness are approximately zero, and these are the conditions we will consider in the following.

A fundamental quantity that determines the expansion of hot dense matter is the speed of sound,

$$
c_{s}^{2}=\left.\frac{\partial P}{\partial \mathcal{E}}\right|_{s / n_{B}},
$$

where the derivative is taken at constant entropy per baryon. Note that at $n_{B}=0$ the speed of sound is simply a function of temperature. The EOS determines how gradients in the energy density profile are translated into pressure gradients. In hydrodynamics, pressure gradients lead to acceleration, and generate collective expansion.

There are several regimes in which we can analytically control the calculation of $c_{s}$. One is the regime of very high temperature, $T \gg T_{c}$. In this regime the running of the coupling is slow, and QCD is approximately scale invariant. This implies that the equation of state is $\mathcal{E}=3 P$ and $c_{s}^{2}=1 / 3$. Perturbative corrections to this result are computable and start at $O\left(\alpha_{s}^{2}\right)$ [80, 81]. At very low temperature and $n_{B}=0$ the pressure is dominated by weakly interacting pions. If pions are massless we also find $c_{s}^{2}=1 / 3$. In practice, pions are non-relativistic for $T \lesssim 100 \mathrm{MeV}$, and the speed of sound approaches that of a classical gas, $c_{s}^{2} \simeq T / m_{\pi}$.

At zero baryon density we then expect the following behavior of the speed of sound: At low temperature the speed of sound is rising towards $c_{s}^{2} \sim 1 / 3$. Near the crossover temperature matter is very compressible and the speed of sound has a minimum. As a function of baryon density, the minimum speed of sound tends to zero as we approach the critical point. At high temperature $c_{s}^{2}$ increases towards the perturbative value $1 / 3$. This behavior implies that a system produced with an initial energy density far above the critical density will initially accelerate quite rapidly, and then coast through the phase transition 
regime. More importantly, a systems produced near the critical energy density will tend to spend some amount of time in the critical regime.

The behavior of the speed of the sound in cold dense matter is quite different. Cold nuclear matter can be understood as a Fermi liquid of protons and neutrons. If we ignore interactions, then the velocity of sound is given by $c_{s}^{2}=k_{F}^{2} /(3 m)$, where the Fermi momentum is defined in terms of the baryon density by $\rho_{B}=$ $2 k_{F}^{3} /\left(3 \pi^{2}\right)$ and we have assumed that the system is isospin symmetric. Near nuclear matter saturation density the ideal Fermi gas speed of sound is $c_{s} \simeq 0.15$. Interactions between nucleons can be described using effective masses and Landau parameters, and lead to modest corrections to the free Fermi gas result. The equation of state at supra-nuclear densities is constrained by neutron star masses and radii. The existence of neutron stars with masses close to two solar masses indicates that the high density EOS is quite stiff, and that the speed of sound at several times nuclear matter density is most likely close to the speed of light [82]. We note that the speed of sound in asymptotically dense quark matter approaches the scale invariant value $c_{s}^{2}=1 / 3$. This implies that whereas the EOS is very soft, and $c_{s}^{2}$ has a minimum, in hadronic matter below the finite temperature transition, the EOS is very stiff, and $c_{s}^{2}$ has a maximum, in hadronic matter below the finite baryon density transition.

\subsection{Lattice $Q C D$}

In the high temperature regime corrections to the equation of state of an ideal quark-gluon plasma can be calculated in perturbation theory. The perturbative expansion is based on the separation of scales $m_{M} \ll m_{D} \ll T$, where $m_{M} \sim g^{2} T$ and $m_{D} \sim g T$ are the effective masses of magnetic and electric modes in the plasma. Strict perturbation theory in $g$ works only for very small values of the coupling constant, $g \lesssim 1$ [83]. However, quasi-particle models that rely on the separation of scales, but not on strict perturbation theory, describe the thermodynamics of the plasma quite well, even for temperatures close to the phase transition to a hadronic gas [84]. Quasi-particle models are quite useful, in particular in connecting equilibrium to non-equilibrium properties of the plasma, but reliable results for the equation of state in the vicinity of $T_{c}$ can only be obtained using numerical calculations on the lattice.

Lattice QCD is based on the euclidean path integral representation of the partition function, see $[86,87]$ for recent reviews. We have

$$
Z(T, \mu, V)=\int \mathcal{D} A_{\mu} \mathcal{D} q_{f} \mathcal{D} \bar{q}_{f} \exp \left(-S_{E}\right)
$$

where $S_{E}$ is the euclidean action

$$
S_{E}=-\int_{0}^{\beta} d \tau \int_{11} d^{3} x \mathcal{L}^{E},
$$




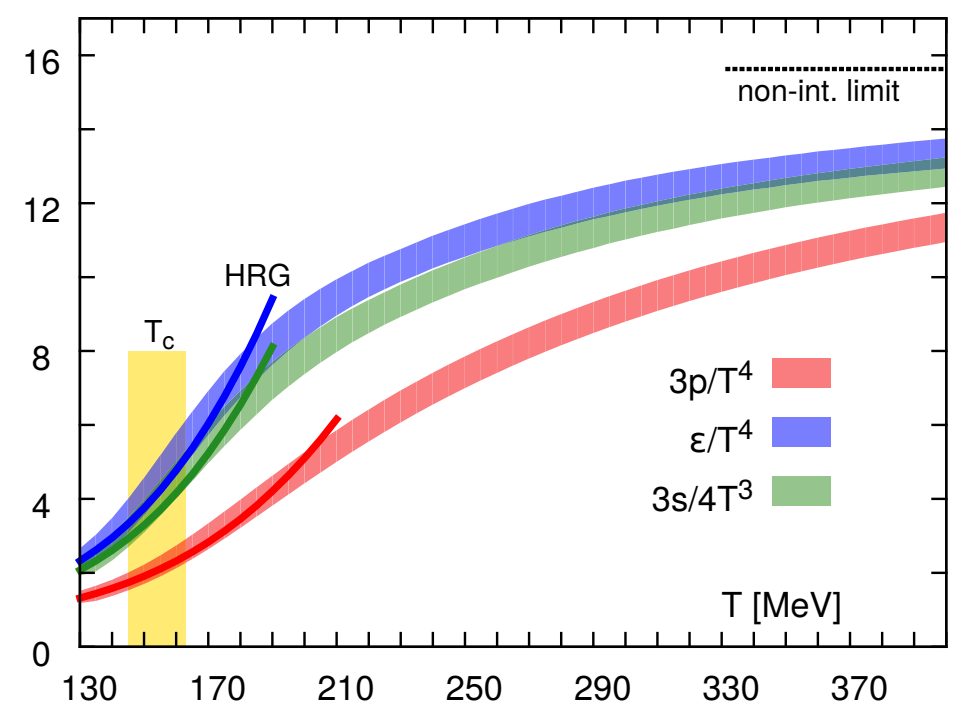

Figure 2: Equation of state of QCD with 2+1 flavors, from [39]. The shows the normalized pressure, energy density, and entropy density as a function of the temperature. The bands indicate systematic and statistical errors. The lines show the prediction of the hadron resonance gas model. The horizontal band at $T_{c}=(154 \pm 9) \mathrm{MeV}$ indicates critical regime. These results are in very good agreement with previous calculations based on a different lattice discretization scheme [85].

$\beta=T^{-1}$ is the inverse temperature and $\mathcal{L}^{E}$ is the euclidean Lagrangian, obtained by analytically continuing Eq. (1) to imaginary time $\tau=i t$. We observe that the temperature enters through the periodicity of the euclidean path integral in the imaginary time direction. Gauge fields and fermions obey periodic and antiperiodic boundary conditions, respectively. The chemical potential couples to the conserved baryon density in the Lagrangian,

$$
\mathcal{L}^{E}(\mu)=\mathcal{L}^{E}(0)+\mu \bar{q}_{f} \gamma_{0} q_{f} .
$$

Following Wilson's original suggestion, the Lagrangian is discretized on an $N_{\tau} \times$ $N_{\sigma}^{3}$ space-time lattice with lattice spacings $a_{\tau}$ and $a_{\sigma}$. In many calculations $a_{\sigma}=$ $a_{\tau}=a$, but this condition is not necessary. In finite temperature calculations we choose $\beta<L$ with $\beta=N_{\tau} a_{\tau}$ and $L=N_{\sigma} a_{\sigma}$, where $V=L^{3}$ is the volume. Eq. (6) provides a lattice definition of the partition function $Z=\exp (-\beta(H-$ $\mu N)$ ). Thermodynamic quantities are determined by taking suitable derivatives, 


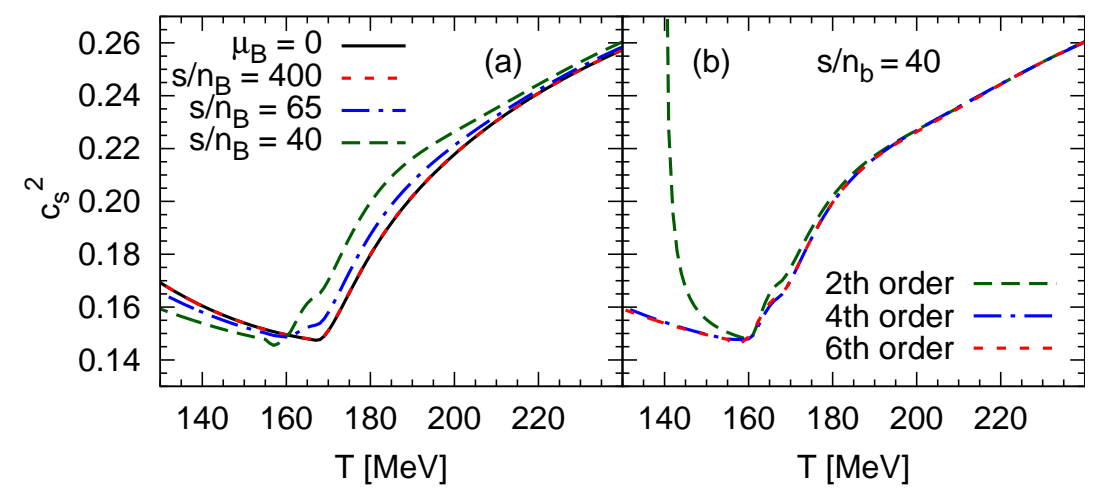

Figure 3: The square of the speed of sound, $c_{s}^{2}$, as a function of temperature on various isentropic curves with constant entropy per baryon. Fig. (a) shows a lattice calculations using a Taylor expansion to explore the regime of finite baryon density finite baryon density [92]. Fig. (b) illustrates the convergence of the method in the case $s / n_{B}=40$.

for example

$$
\begin{aligned}
\mathcal{E} & =-\left.\frac{1}{V} \frac{\partial \log Z}{\partial \beta}\right|_{\beta \mu}, \\
n_{B} & =\left.\frac{1}{\beta V} \frac{\partial \log Z}{\partial \mu}\right|_{\beta} .
\end{aligned}
$$

The gauge fields are discretized on links and the fermion fields reside on sites. This allows the gauge invariance of QCD to be maintained exactly, even on a finite lattice, but Lorentz invariance is only restored in the continuum limit. We note that because of classical scale invariance the massless QCD action is independent of $a$. The continuum limit is taken by adjusting the bare coupling at the scale of the lattice spacing according to asymptotic freedom, see Eq. (4). In practical calculations the lattice spacing is not quite small enough to ensure the accuracy of this method, and more sophisticated scale setting procedures are used [86, 87].

Formally, the integration over the fermion fields can be performed exactly, resulting in the determinant of the Dirac operator $\operatorname{det}\left(M\left(A_{\mu}, \mu\right)\right)$. Several methods exist for discretizing the Dirac operator $M$, and for sampling the determinant. Different discretization schemes differ in the degree to which chiral symmetry is maintained on a finite lattice. The original formulation due to Wilson [88] preserves no chiral symmetry, the staggered Fermion scheme [89] maintains a subset of the full chiral symmetry, while the domain wall [90] and overlap methods [91] aim to preserve the full chiral symmetry on a discrete lattice.

A second issue with fermions is that for $\mu \neq 0$ the fermion determinant is no longer real, so that standard importance sampling methods fail. This is the 
"sign" problem already mentioned in Section 2.2. There are many attempts to find direct solutions to the sign problem, but at this time the only regime in which controlled calculations are feasible is the regime of small $\mu$ and high $T$. In this region the partition function can be expanded in a Taylor series in $\mu / T$. The corresponding expansion coefficients are generalized susceptibilities that can be determined from lattice simulations at zero chemical potential. The susceptibilities not only determine the equation of state at finite baryon density, but also control fluctuations of conserved charges as explained in Section 5.

As examples of lattice results that are central to the analysis of heavy ion collisions we show a calculation of equation of state at $\mu=0$ in Fig. (2 [39], and the speed of sound for several values of $\mu$ in Fig. (3) $[92,85,39]$. We observe that the energy density, pressure, and entropy density, normalized to suitable powers of $T$, vary rapidly in the critical regime defined by fluctuations of the chiral order parameter. We also note that the agreement with the hadron resonance gas model is very good up to temperature $T \lesssim 180 \mathrm{MeV}$. Finally, we observe that the rise of $\mathcal{E}, P$ and $s$ towards the perturbative limit is quite slow. In particular, even at temperatures as large as $T=400 \mathrm{MeV}$, the pressure remains about $25 \%$ below the Stefan-Boltzmann limit.

The speed of sound at $\mu=0$ was determined from direct lattice simulations of $P(T)$. The results at non-zero baryon density were obtained using the Taylor expansion method. We observe that $c_{s}^{2}$ indeed shows the expected behavior, a soft point near the phase transitions where $c_{s}^{2} \simeq 0.15$, followed by a gradual rise towards $c_{s}^{2}=1 / 3$. In the regime accessible with Taylor expansions the dependence of $c_{s}^{2}$ on $n_{B}$ is not very pronounced. The main effect is a slight reduction in the temperature of the softest point, corresponding to the curvature of the phase transition line in the $T-\mu$ plane.

\subsection{Lattice QCD: Frontiers and challenges}

The equation of state in three flavor QCD with physical quark masses and vanishing baryon density is now fairly well established, but many new challenges for lattice QCD have emerged. One challenge is clearly to extend calculations of the EOS into the regime of finite baryon density and to locate or exclude the presence of a critical point. In addition to methods that are restricted to the regime $\mu \lesssim \pi T$, a number of proposals to explore QCD at high baryon density are being pursued. This includes new approaches, like integration over Lefshetz thimbles [93, 94], as well as novel variants of old approaches, like the complex Langevin method [95, 96], or the use of dual variables [97]. The ultimate promise of these methods is still unclear, but the central importance of the sign problem to computational physics continues to attract new ideas.

Some progress has also been achieved in a different area, the calculation of near-equilibrium real time properties of the plasma using response functions [98]. 
The prototypical example is the calculation of the shear viscosity using the retarded correlation function of the stress tensor $T_{x y}$,

$$
G_{R}^{x y, x y}(\omega, \vec{k})=-i \int d t \int d^{3} x e^{i(\omega t-\vec{k} \cdot \vec{x})} \Theta(t)\left\langle\left[T^{x y}(\vec{x}, t), T^{x y}(0,0)\right]\right\rangle,
$$

The associated spectral function is defined by $\rho(\omega, \vec{k})=-\operatorname{Im} G_{R}(\omega, \vec{k})$. As we will explain in more detail in Section 4.2.2 the imaginary part of the retarded correlator is a measure of dissipation. Matching the correlation function from linear response theory to the hydrodynamic correlator gives the Kubo relation

$$
\eta=\lim _{\omega \rightarrow 0} \lim _{k \rightarrow 0} \frac{\rho^{x y, x y}(\omega, \vec{k})}{\omega} .
$$

The formula for the bulk viscosity involves the trace of the energy momentum tensor

$$
\zeta=\frac{1}{9} \lim _{\omega \rightarrow 0} \lim _{k \rightarrow 0} \frac{\rho^{i i, j j}(\omega, \vec{k})}{\omega},
$$

and analogous results can be derived for the thermal conductivity and diffusion constants.

The spectral function contains information about the physical excitations that carry the response. Lattice calculations are based on the relation between the spectral function and the Matsubara (imaginary energy) correlation function

$$
G_{E}\left(i \omega_{n}\right)=\int \frac{d \omega}{2 \pi} \frac{\rho(\omega)}{\omega-i \omega_{n}}
$$

where $\omega_{n}=2 \pi n T$ is the Matsubara frequency. The imaginary time correlation function is given by

$$
G_{E}(\tau)=\int \frac{d \omega}{2 \pi} K(\omega, \tau) \rho(\omega)
$$

where the kernel $K(\omega, \tau)$ is defined by

$$
K(\omega, \tau)=\frac{\cosh [\omega(\tau-1 /(2 T))]}{\sinh [\omega /(2 T)]}=\left[1+n_{B}(\omega)\right] e^{-\omega \tau}+n_{B}(\omega) e^{\omega \tau},
$$

and $n_{B}(\omega)$ is the Bose distribution function. The imaginary time correlation function (15) was determined in a number of lattice studies [99, 100, 101, 102]. The basic approach for extracting transport properties is to compute $G_{E}(\tau)$ numerically, invert the integral transform in Eq. (15) to obtain $\rho(\omega)$, and finally obtain the transport coefficient from the limit $\omega \rightarrow 0$ of the spectral function. 
The difficulty is that $G_{E}(\tau)$ is typically only known on a small number of lattice sites, and that the imaginary time correlator is not very sensitive to the slope of the spectral function at low energy. Many recent calculations make use of the maximum entropy method to obtain numerically stable spectral functions and reliable error estimates $[103,104]$. It was also observed that one can minimize the contribution from continuum states to the imaginary time Green function by studying the correlators of conserved charges, energy and momentum density, at non-zero spatial momentum $[105,106]$. In physical terms this means that one can extract the viscosity from the sound pole rather than the shear pole in the retarded correlator.

Pioneering calculations of the shear viscosity were performed by Karsch and Wyld [99]. More recently, the problem of determining shear and and bulk viscosity near $T_{c}$ was revisited by Meyer $[100,106]$. He finds $\eta / s=0.102(56)$ and $\zeta / s=0.065(17)$ at $T=1.24 T_{c}$. The shear viscosity is only weakly temperature dependent, but bulk viscosity grows strongly near $T_{c}$, and becomes very small at large temperature. The value of $\eta / s$ is consistent with the experimental determinations discussed in Section 4.3, and the proposed holographic bound $\eta / s=1 /(4 \pi)[107]$. An interesting aspect of these calculations is that it is easier to numerically determine a small shear viscosity as compared to a large one. In weak coupling $\eta / s$ is large (see Eq. 32), but this result is encoded in a very narrow peak in the spectral function, which is hard to resolve numerically. A small shear viscosity, on the other hand, corresponds to a very smooth spectral function, which is much easier to reconstruct. This implies that the reported lattice determinations of $\eta / s$ near $T_{c}$ may well be reliable, but that it is also difficult to demonstrate the accuracy of the method by studying the weak coupling limit $T \gg T_{c}$. Recent calculations of spectral functions have focused on other observables, in particular the heavy quark diffusion constant, the dilepton spectral function, and the spectrum of charmonia with different quantum numbers, see [87] for an overview.

\section{Hadrons with $(u, d, s)$ quarks}

Hadron production in ultra-relativistic nucleus-nucleus collisions has been studied now for nearly 30 years. The first successful description of a comprehensive set of data within the framework of statistical hadronization was achieved in 1994 for data from the Brookhaven AGS and $\mathrm{Si}+\mathrm{Au}(\mathrm{Pb})$ collisions [19]. The same approach was applied to more data soon thereafter. In this context we also point to an early article by Gerry Brown and co-workers [51], where a thermal approach was successfully used to describe the influence of resonance decays on pion spectra. Excellent surveys of the data from the pre-LHC era, together with an analysis and interpretation in the framework of the statistical hadronization 


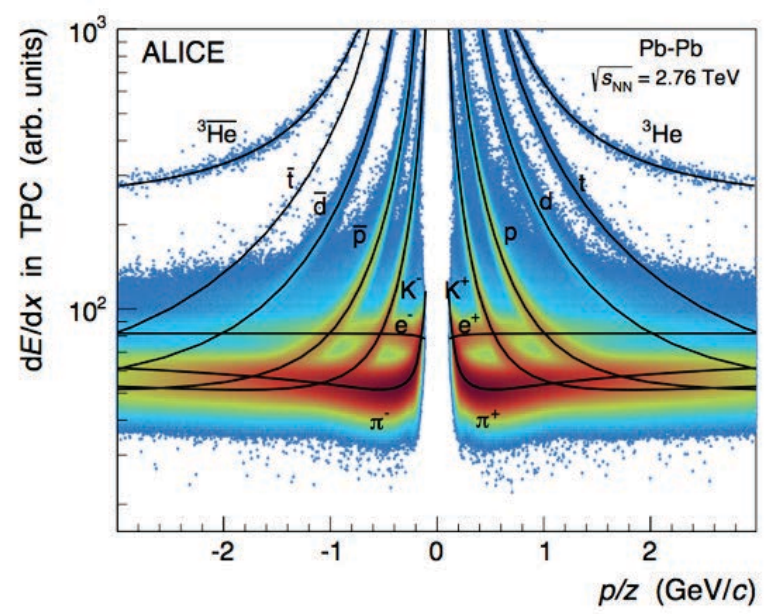

Figure 4: Particle identification in the ALICE experiment via the specific energy loss and momentum measurement in the ALICE TPC and inner tracking system (figure taken from $[108])$.

model, can be found in $[109,110,111]$. The first three years of data taking at the CERN Large Hadron Collider (LHC) have brought a wealth of new, high precision data on hadron production in ultra-relativistic nuclear collisions at the $\mathrm{TeV}$ energy scale. As an example of the data quality we show, in Figs. 4 and 5 , the particle identification with the ALICE TPC and the reconstructed mass distributions for strange baryons in $\mathrm{Pb}-\mathrm{Pb}$ collisions with ALICE at the LHC.

A compilation of the available data on hadron production at mid-rapidity in central nucleus-nucleus collisions is presented in Fig. 6. This comprehensive plot contains the work of essentially all large collaborations in the field of ultrarelativistic heavy ion physics, performed over a period of approximately 30 years. Some regularities are obvious: At lower energies protons from the colliding nuclei dominate the yield at mid-rapidity while all produced particles are strongly suppressed. With increasing energy baryon pair production becomes more and more dominant, and the charge and baryon number in the colliding nuclei (fragmentation regions) becomes irrelevant at mid-rapidity. As a consequence, at LHC energies, the central fireball formed in the collisions contains equal amounts of matter and anti-matter: Big Bang matter produced in the laboratory ${ }^{1}$. It is this rich data sample on which all further interpretations are based. Two of us (pbm and js) remember discussing versions of this plot with Gerry Brown during the early years of RHIC operation while trying to convince him (successfully,

\footnotetext{
${ }^{1}$ Big Bang matter contains, in addition to hadrons, also leptons (including neutrinos) and photons and, at very high temperatures, electro-weak bosons in equilibrium.
} 

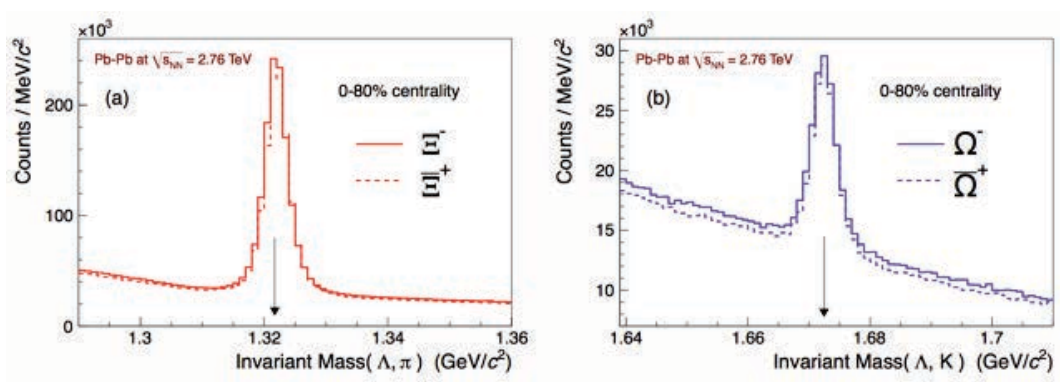

Figure 5: Reconstruction of multi-strange baryons produced in central $\mathrm{Pb}-\mathrm{Pb}$ collisions with ALICE at the LHC via the invariant mass of their weak decay products (figure taken from $[112])$.

we believe) to focus his mind again on the beautiful phenomenology of particle production in ultra-relativistic nuclear collisions.

The data, of which some systematics is shown in Fig. 6, can be economically and concisely described over the whole energy range in the framework of the statistical hadronization model. This thermal or statistical hadronization model [114] (we will use the term synonymously here) describes a snapshot of the collision, namely the chemical freeze-out, which is assumed to be driven by rapid changes in energy and entropy density near the phase boundary [115]. The fireball formed in the collision is assumed to be in chemical equilibrium when the dramatic changes in density near the phase boundary lead to (nearly) simultaneous freeze-out of all hadrons at the chemical freeze-out temperature $T$ and baryo-chemical potential $\mu_{b}$. The energy dependence of $T$ and $\mu_{b}$ and of the rapidity density of charged pions determine the thermal parameters $T, \mu_{b}$ and $V$ and, hence, the rapidity density of all hadron species. In general, the precision of this description is on the order of $10 \%$. Due to the data sets available, the energy dependence of the thermal parameters is measured at discrete energies and interpolated in between, see below.

This approach provides a phenomenological link between the data and the QCD phase diagram shown in Fig. 1, a link surmised a long time ago $[5,116]$ but explored and discussed in quantitative detail only more recently [117, 118, 119, $115,111,120,121]$. In this review we use the most recent data and the latest update of the model as described in [122].

We note that, for the first time, the data obtained by the ALICE collaboration at the LHC are corrected in hardware for feed-down from weakly decaying resonances via the use of the excellent ALICE inner tracking detector, see [123]. Consequently, for a description of ALICE data no feed-down correction is applied to the thermal model calculations. For analysis of the data from the RHIC, SPS and AGS accelerators, feeding from weak decays needs to be taken into account. 


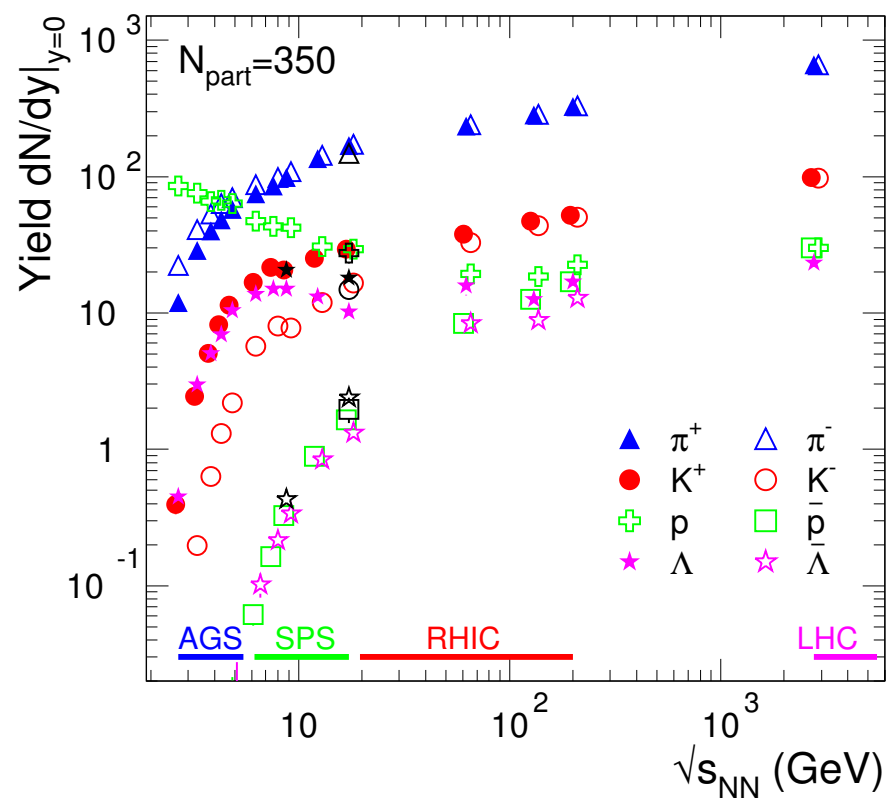

Figure 6: Energy dependence of the rapidity density for identified hadrons produced in central nucleus-nucleus collisions. Figure taken from $[109,113]$. The colliding systems are either $\mathrm{Pb}-\mathrm{Pb}$ or $\mathrm{Au}-\mathrm{Au}$ and central collisions are selected by the requirement of at least 350 participating nucleons in each collision.

For details of this procedure see, e.g., $[109,111]$. The uncertainties resulting from this correction lead to significantly increased uncertainties in the data from RHIC and the lower energy accelerators compared to those from the LHC.

Good fits of the measurements are achieved with the thermal model [114] with 3 parameters: Temperature $T$, baryochemical potential $\mu_{B}$, and volume $V$, as shown in Fig. 7 for the fit of data at the LHC [122, 124]. Remarkably, multiply-strange hyperons and light nuclei and (hyper)nuclei are well described by the model. At LHC energy, the baryochemical potential turns out be zero within uncertainties, implying [126] equal production of matter and antimatter at the LHC [127]. Note that also loosely bound systems such as the deuteron (with binding energy $E_{b}=2.23 \mathrm{MeV}$ ) and hypertriton (binding energy $E_{b}=2.35$ $\mathrm{MeV}, \Lambda$ separation energy $S_{\Lambda}=0.13 \mathrm{MeV}$ ) are well reproduced for a $T$ value of $156 \mathrm{MeV}$, i.e. $T \gg E_{b} \gg S_{\Lambda}$. The rates for such loosely bound systems are indeed fixed near the phase boundary, implying that the expansion after chemical freezeout is isentropic. It is then immaterial for the calculation whether the (hyper) nuclei are droplets of quark matter [128] or are formed via nucleon (and hyperon) coalescence. 


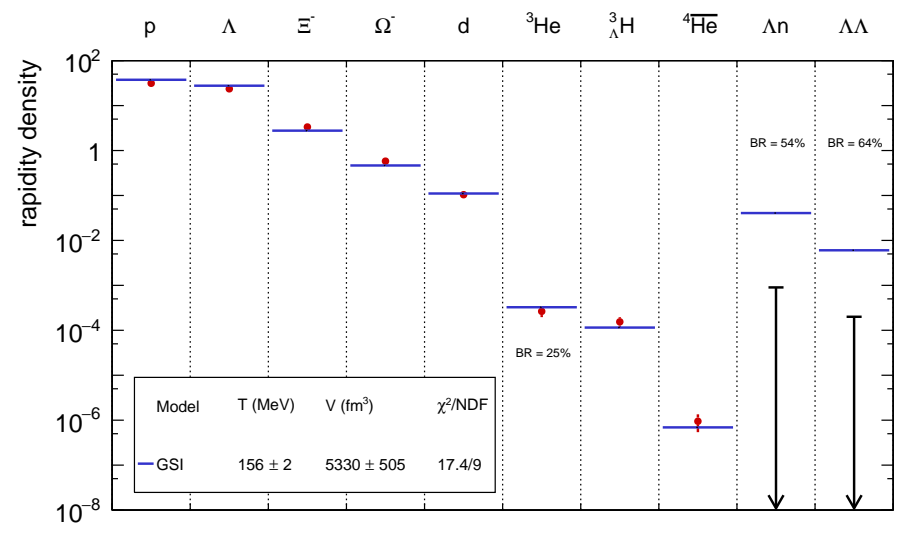

Figure 7: Measured hadron abundances in comparison with thermal model calculations for the best fit to ALICE data [124] for central Pb-Pb collisions at the LHC. Plotted are the "total" thermal model yields, including all contributions from strong decays of high-mass resonances (for the $\Lambda$ hyperon, the contribution from the electromagnetic decay $\Sigma^{0} \rightarrow \Lambda \gamma$, which cannot be resolved experimentally, is also included) (figure taken from [125]).

An interesting and to-date not fully appreciated (or understood) outcome of these thermal model analyses is that the best fits are obtained if all hadron masses are kept at their vacuum values irrespective of the values of $T$ and $\mu_{b}$. As discussed below, the chemical freeze-out temperature $T$ approaches, for centerof-mass energies $\sqrt{s_{n n}}>10 \mathrm{GeV}$ closely the values predicted from lattice QCD calculations for the (pseudo-)critical temperature of the quark-hadron transition, yet no deviation from the vacuum mass scenario is seen.

A remarkable outcome of these fits is that $T$ increases with increasing energy and decreasing $\mu_{B}$ from about $50 \mathrm{MeV}$ to about $160 \mathrm{MeV}$, where it exhibits, see Fig. 8, a saturation for $\sqrt{s_{n n}}>10 \mathrm{GeV}$ and $\mu_{B} \lesssim 300 \mathrm{MeV}$. The freeze-out points can be put together for an experimental version of the phase diagram, see Fig. 9. An interpretation of the saturation of the freeze-out temperature was put forward in [120], based on the conjecture that the chemical freeze-out temperature is the hadronization temperature [111], and therefore probes the QCD phase boundary. The proposal is that the two regimes in the phase diagram, see Fig. 1 and Fig. 9, that of approximately constant $T$ for small $\mu_{B}$ values, and that of the strong increase in $T$ at low energy and large $\mu_{B}$, may reflect the existence of a triple point in the QCD phase diagram [120], see Section 2. Various criteria for the chemical freeze-out were proposed $[129,130]$. In our understanding it is linked to the rapid drop in energy, entropy, and particle densities near the pseudocritical temperature [115], leading first to equilibrium hadron population at or just below $T_{C}$ and then to rapid fall-out of equilibrium (i.e. freeze-out).

In Fig. 10 we demonstrate that the thermal model approach can be success- 

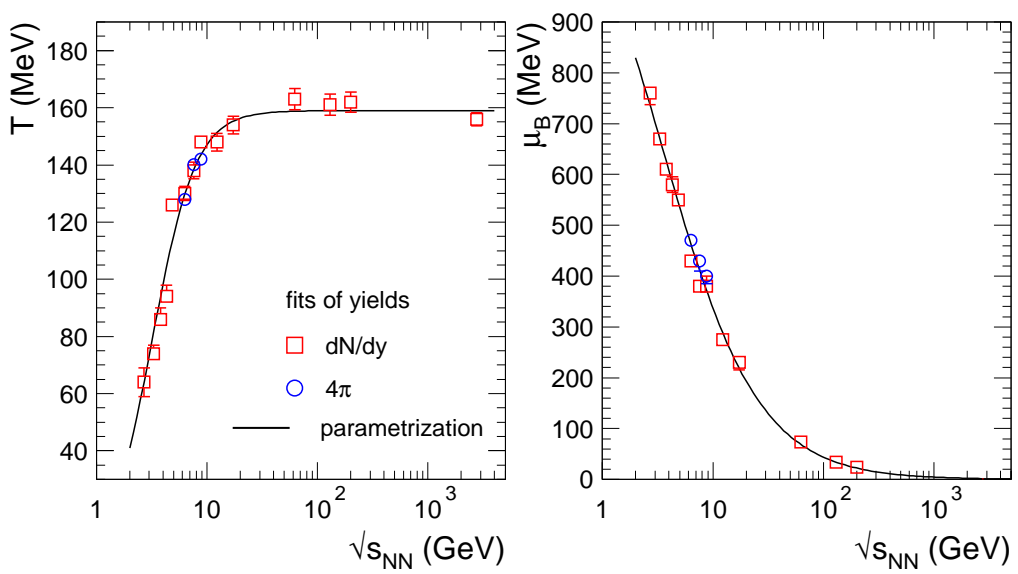

Figure 8: Energy dependence of the thermal model parameters $T$ and $\mu_{b}$ [109] updated to include the most recent LHC results. For more details see text. Figure taken from [113].

fully used to reproduce, over the full energy range where data have been measured, the ratios of production yields for various hadron species. The calculations are being performed with parametrizations for the energy dependence of $T$ and $\mu_{B}$ as obtained in Ref. [111] and updated for [122]. The striking energy dependence of $T$ vs $\sqrt{s}$ is shown in Fig. 8 along with the very smooth decrease with energy of $\mu_{b}$.

The non-monotonic dependence on energy of the $K^{+} / \pi^{+}$yield ratio was originally proposed as a signature [135], and the measurement by the NA49 collaboration taken as evidence [136] for the onset of deconfinement. However, the results including the rather pronounced maximum near $\sqrt{s_{n n}}=8 \mathrm{GeV}$ are well understood within the above described framework of the thermal model [111], as shown in Fig. 10 (right panel). Based on this success, the thermal model predictions provide a reliable guidance for experimental searches for other exotic nuclei [137].

The phenomenological phase diagram obtained from these hadron yield analyses within the statistical hadronization model is shown in Fig. 9. Each point corresponds to a fit of hadron yields in central $\mathrm{Au}-\mathrm{Au}$ or $\mathrm{Pb}-\mathrm{Pb}$ collisions at a given collision energy. The agreement between the results from several independent analyses $[111,132,133,134]$ is remarkable. In some cases $[132,133,134]$ an additional ad-hoc fit parameter, the 'strangeness suppression' factor $\gamma_{s}$, is used to search for a departure from equilibrium of hadrons containing strange quarks. Values of $\gamma_{s}$ (slightly) below unity are found although no statistically significant improvement in the fit is obtained, especially when the fit is restricted to data at mid-rapidity. An approach with more non-equilibrium parameters [138, 139] also 


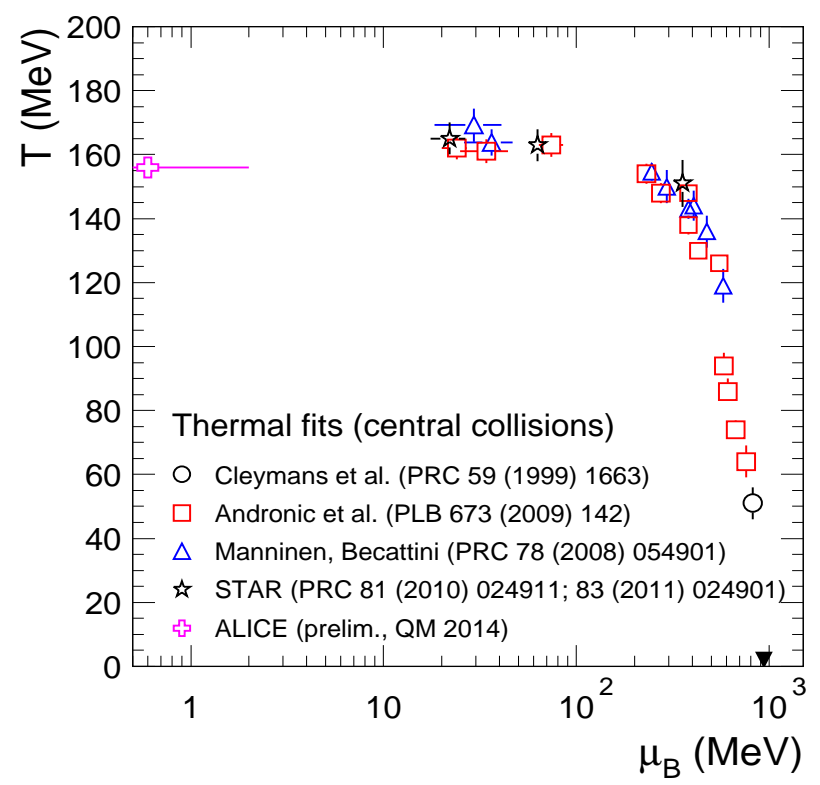

Figure 9: The phase diagram of strongly interacting matter with the points representing the thermal fits of hadron yields at various collision energies [131, 111, 132, 133, 134, 124]. For the LHC, $\mu_{B}=0$ is the value resulting from the fit. In the plot, a value of $0.6 \mathrm{MeV}$ is used here for adaptation to the logarithmic scale. The down-pointing triangle indicates the baryo-chemical potential of bound nuclei, i.e. $\mu_{b}=931.4 \mathrm{MeV}$.

does not lead to significant improvements but results in a non-monotonic energy dependence and generally decreased values for $T$. Fits considering a spread in $T$ and $\mu_{B}$ were also performed [140] and are currently again under consideration because of the cross-over nature of the transition.

The thermal model inherently provides information on the underlying thermodynamic quantities characterizing the state of the fireball under consideration. While the absolute yields are insensitive to the question whether or not repulsive interactions are considered (to first order they are renormalized via the volume) quantities like energy density, pressure, entropy density and particle density significantly depend on the choice of interaction. Here we follow the approach taken in [141]. There, the repulsive part of the interactions is taken into account via excluded volumes. Each meson or baryon 'excludes' a spherical volume with radius $R_{\text {meson }}$ or $R_{\text {baryon }}$. The corresponding interaction correction is then obtained following the procedure of [142]. The derived thermodynamic quantities are shown as a function of energy in Fig. 11 for various values of the excluded volume radii. At LHC energy, corresponding to the "limiting temperature" $T_{\text {lim }}=159 \mathrm{MeV}$, the following values are obtained for $R_{\text {meson }}=R_{\text {baryon }}=0.3 \mathrm{fm}$ : pressure $P \simeq 60$ 

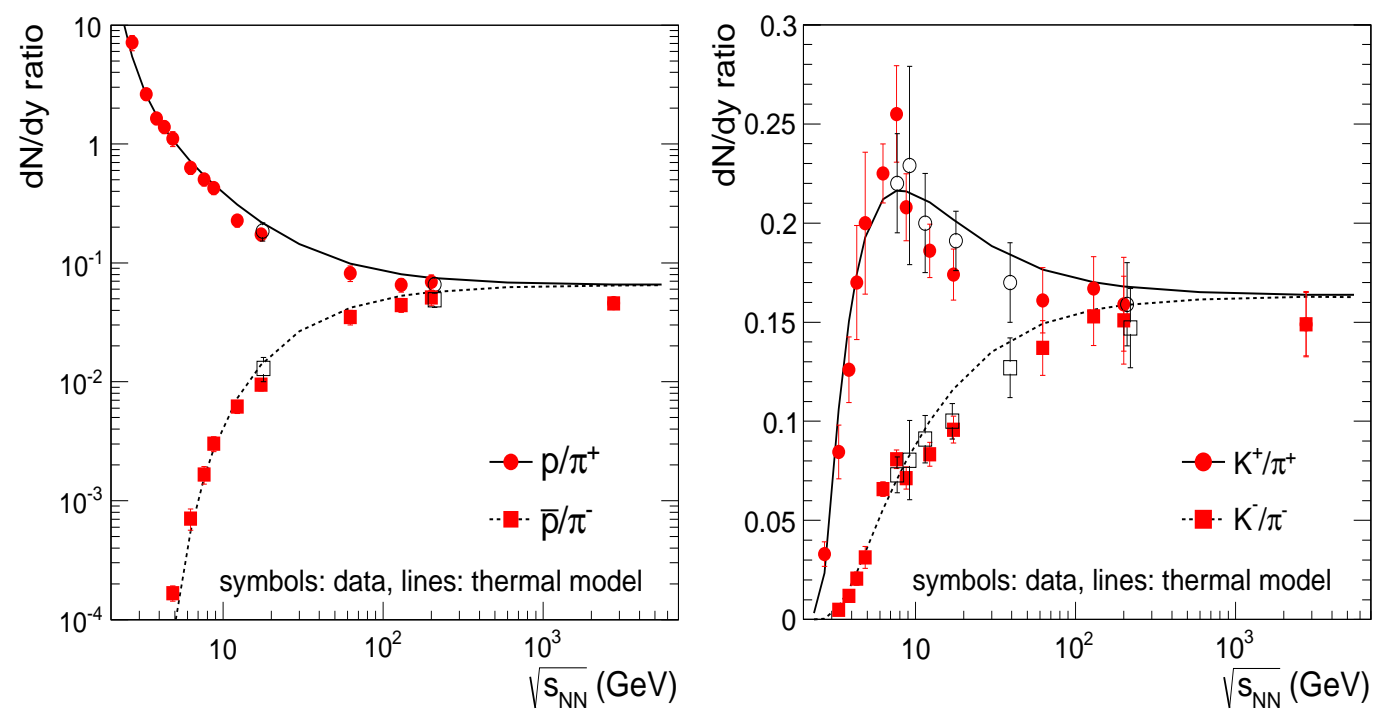

Figure 10: Collision energy dependence of ratios of yields of protons and antiprotons (left panel) and of kaons (right panel) to yields of pions (figure taken from [113]. The symbols are data, the lines are thermal model calculations for energy-dependent parametrizations of $T$ and $\mu_{B}$ (as in Ref. [111]).

$\mathrm{MeV} / \mathrm{fm}^{3}$, energy density $\varepsilon \simeq 330 \mathrm{MeV} / \mathrm{fm}^{3}$, entropy density $s=2.4 \mathrm{fm}^{-3}$, density of mesons $n_{m}=0.26 \mathrm{fm}^{-3}$, total baryon density (particles plus antiparticles) $n_{B+\bar{B}}=0.06 \mathrm{fm}^{-3}$.

While the fit quality reported in Fig. 7 is impressive indeed, we would like to point out that there is currently a $2.7 \sigma$ excess, not visible on the log plot in the figure, of the calculated thermal model yields over the data for protons and antiprotons. Given the overall excellent agreement at LHC energy, this discrepancy led to a number of theoretical investigations, mostly stressing the possible importance of the role of interactions after chemical freeze-out [143] (in the hybrid model of Ref. [143] higher $T$ values are obtained for the LHC case); also the effect of a possible extension of the hadronic mass spectrum beyond the currently established hadron states $[122,144,145]$ was discussed. To date, there is no consistent explanation of the apparent 'LHC proton puzzle', especially also considering the excellent description of light nuclei and antinuclei as well as the situation in the multi-strange baryon sector, where final state annihilation, should it exist, would also be visible. The connection to fit results for data from $\mathrm{e}^{+} \mathrm{e}^{-}$ (see Ref. [146] and references therein) and in elementary hadronic collisions [147] remains also to be better understood.

The results reported up to this point only deal with the mean number of hadrons produced for a given collision centrality, i.e. with the first moment of 


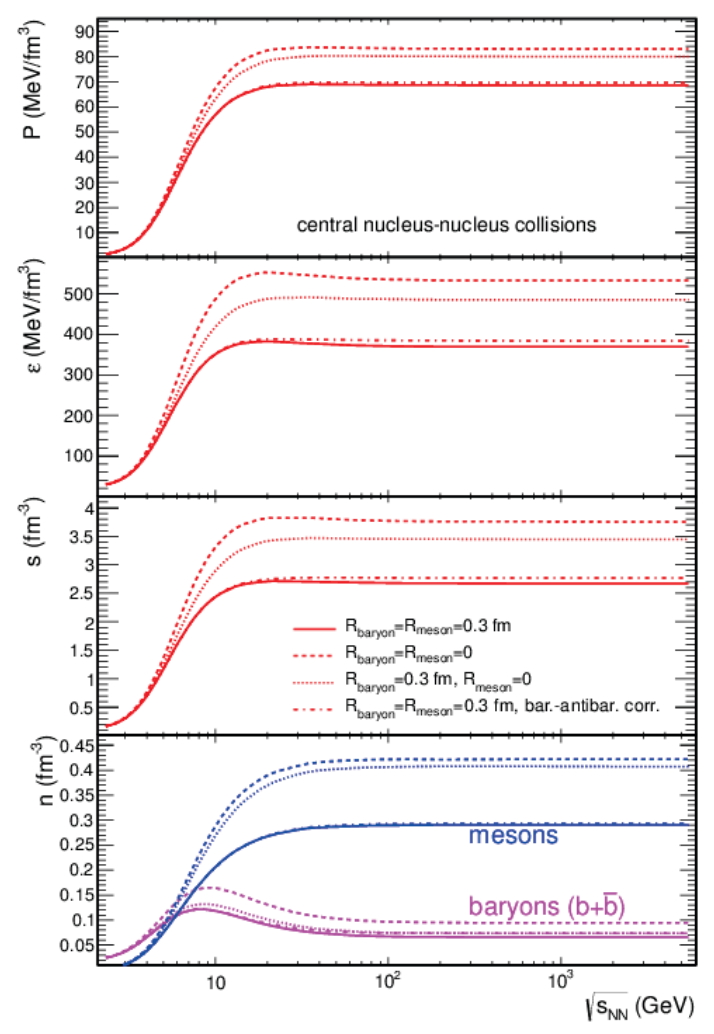

Figure 11: Energy dependence of thermodynamic variables resulting from the thermal model analysis. The various curves correspond to different treatment of the excluded volume correction, which is used to model repulsive interactions in the hadron resonance gas. Figure taken from [141].

the thermal model distributions. It is clearly interesting to extend these studies into the measurement of higher moments and to investigate whether these can also be described within the framework of the grand-canonical hadron resonance gas, i.e. the thermal model described here. This will be discussed in detail in Section 5.3.

\section{Fluid dynamics and collective flow}

\subsection{Introduction}

The non-equilibrium evolution of a heavy ion collision, beginning from the initial production of partons or fields, the possible formation of a quark-gluon plasma and its subsequent expansion, to a hadronization stage and the final decoupling, is clearly a very complicated process. However, the situation simplifies 
greatly if the interaction in the initial state is strong, and rapid local thermalization occurs. In this case microscopic details of the state of the system are not relevant, and the dynamics is completely determined by the distribution of energy, momentum, and baryon density. The time evolution of these quantities is governed by the corresponding conservation laws, as encoded in the equations of fluid dynamics. The reason that conserved charges are special is that generic observables can relax locally, on a microscopic time scale, while conserved quantities can only relax by collective motion or diffusion, which takes place on a macroscopic time scale. Fluid dynamics depends on a small number of equilibrium and near-equilibrium parameters, most notably the equation of state and the transport coefficients of the fluid, see $[148,149,150,151,152]$ for recent reviews. The collective expansion of the hot and dense matter created in the collision therefore constrains these parameters.

In the late stages of the collision matter hadronizes, interactions become weak, and the fluid dynamic description must break down. However, because the conversion of a fluid to weakly interacting particles is a local process, the collective flow pattern established in the fluid dynamic stage is robust. Furthermore, if the breakdown of fluid dynamics occurs in a regime in which kinetic theory based on hadronic quasi-particles is valid, then a more microscopic description is possible and the combination of fluid dynamics and kinetic theory can be used to predict particle spectra, and the flow of identified particles.

\subsection{Relativistic fluid dynamics}

In the ultra-relativistic regime we can neglect the baryon density and the conserved densities are the energy density $\mathcal{E}$ and the momentum density $\vec{\pi}$. These two hydrodynamic variables can be embedded in the energy momentum tensor $T_{\mu \nu}$, where $T^{00}=\mathcal{E}$ and $T^{0 i}=\pi^{i}$. Conservation of energy and momentum is expressed by the equation

$$
\partial^{\mu} T_{\mu \nu}=0
$$

In order to solve this equation we have to determine the remaining components of $T_{\mu \nu}$. The basic idea is to make use of the fact that hydrodynamics is a macroscopic, coarse-grained, description of the underlying microscopic dynamics. This implies that we can systematically expand the currents in gradients of the fluid dynamic variables. The leading order expression contains no gradients, and is completely fixed by symmetries. The basic observation is that in the fluid rest frame, $T_{\mu \nu}=\operatorname{diag}(\mathcal{E}, P, P, P)$. In a general frame

$$
T_{\mu \nu}^{(0)}=(\mathcal{E}+P) u_{\mu} u_{\nu}+P g_{\mu \nu},
$$

where we have introduced the fluid velocity $u^{\mu}$, and we use the convention $u^{2}=$ -1 . The fluid dynamic equations $\partial^{\mu} T_{\mu \nu}=0$ close once we provide an equation of state, $P=P(\mathcal{E})$. 
Equation (17) is the relativistic Euler equation. We can split this equation into longitudinal and transverse parts using the projectors

$$
\Delta_{\mu \nu}^{\|}=-u_{\mu} u_{\nu}, \quad \Delta_{\mu \nu}=g_{\mu \nu}+u_{\mu} u_{\nu} .
$$

The longitudinal and transverse projections of $\partial^{\mu} T_{\mu \nu}=0$ can be viewed as the equation of energy (or entropy) conservation, and the relativistic Euler equation. We get $\partial^{\mu}\left(s u_{\mu}\right)=0$ and

$$
D u_{\mu}=-\frac{1}{\mathcal{E}+P} \partial_{\mu}^{\perp} P
$$

where $D=u^{\mu} \partial_{\mu}$ and $\partial_{\mu}^{\perp}=\Delta_{\mu \nu} \partial^{\nu}$. Eq. (20) has the same structure as the nonrelativistic Euler equation, where $D$ plays the role of the comoving derivative, and $\mathcal{E}+P$ is the inertia of the fluid.

Gradient corrections to Eq. (18) are important for a number of reasons. The most important is that the Euler equation is exactly time reversal invariant, and no entropy is produced (there is an exception to this statement if shocks are present). Gradient terms, on the other hand, violate time reversal invariance and lead to the production of entropy. This implies, in particular, that even if gradient terms are small at any given time, their effects can exponentiate and the late time flow is qualitatively different.

In order to identify gradient corrections to $T_{\mu \nu}$ we have to define the fluid velocity more carefully. In the ultra-relativistic domain we can define $u^{\mu}$ through the condition $u^{\mu} T_{\mu \nu}=\mathcal{E} u_{\nu}$. This relation, called the Landau frame condition, implies that the energy current in the rest frame does not receive any dissipative corrections, $\left.T_{0 i}\right|_{r f}=0$. In the non-relativistic domain it is more natural to define the fluid velocity in terms of the baryon current $j_{B}^{\mu}=u^{\mu} n_{B}$. This condition, called the Eckart frame, implies that there are no dissipative corrections to the baryon current but allows gradient corrections to the energy current in the rest frame of the fluid. Physically, the two frames are of course equivalent. For example, heat conduction appears as a correction to the energy current in the Eckart frame, and as a correction to the baryon current in the Landau frame.

After these preliminaries, we can state the possible first order gradient terms in the stress tensor. We have

$$
\delta T_{\mu \nu}^{(1)}=-\eta \sigma_{\mu \nu}-\zeta g_{\mu \nu}(\partial \cdot u),
$$

where $\eta$ is the shear viscosity, $\zeta$ is the bulk viscosity, and we have defined

$$
\sigma^{\mu \nu}=\Delta^{\mu \alpha} \Delta^{\nu \beta}\left(\partial_{\alpha} u_{\beta}+\partial_{\beta} u_{\alpha}-\frac{2}{3} \eta_{\alpha \beta} \partial \cdot u\right)
$$

where $\Delta_{\mu \nu}$ is the projector defined in Eq. (19). This definition ensures that in the local rest frame there is no dissipative correction to the energy current, and only 
the momentum density current is modified. This modification describes friction and viscous heating in the fluid. We also note that in a scale invariant fluid the bulk viscosity vanishes. Kinetic theory suggests that the shear viscosity scales as

$$
\eta \sim s T \tau_{\text {coll }},
$$

where $\tau_{\text {coll }}$ is a typical collision time scale. In perturbative QCD $\tau_{\text {coll }} \sim 1 /\left(g^{4} T\right)$, see Section 4.2.2. Bulk viscosity is controlled by the same collision rate, but is also sensitive to deviations of the equation of state from exact scale invariance, $\mathcal{E} \neq 3 P$. A simple estimate is $\zeta \sim(\mathcal{E}-3 P)^{2} \eta[81,153]$, but deviations from this relation may appear in the strong coupling limit. The factor $\mathcal{E}-3 P$ occurs squared because deviations from scale invariance are needed for collisions to build up a scalar contribution to non-equilibrium distribution functions, and they are also required for the non-equilibrium distribution function to feed into a nonequilibrium pressure term.

The formalism presented in this Section is well established, and has been known for many years. However, before a flurry of activity triggered by the first RHIC data led to a re-evaluation of the theory, it was widely believed that the relativistic Navier-Stokes equation is unstable and probably ill-defined, and that there is no hope for the gradient expansion to convergence in a relativistic heavy ion collision (see $[154,155]$ for some notable exceptions). In the following we will describe how our understanding of relativistic fluid dynamics has evolved in response to both the data, and to progress in the theory of relativistic fluid dynamics.

\subsubsection{Consistency of the Navier-Stokes equation}

Once the equations of fluid dynamics are determined it is natural to study the motion of small fluctuations on a fixed background flow [156]. One obvious excitation is sound. In a fluid at rest longitudinal sound waves are propagating with the speed of sound $c_{s}$. A second, transverse, excitation is a diffusive shear wave. The dispersion relation of a shear wave is $\omega=i \nu k^{2}$, where $\nu=\eta /(s T)$. Consequently the "speed" $(\partial|\omega|) /(\partial k)$ of a diffusive wave is $[150]$

$$
v_{D} \simeq 2 \nu k
$$

and a very sharp diffusive front can move at arbitrarily high velocity. This implies that the relativistic Navier-Stokes equation is acausal for $k \gtrsim s T /(2 \eta)$. It was also found that in this regime the Navier-Stokes equation has unstable modes [157].

We emphasize that there is nothing fundamentally wrong here: Fluid dynamics is an effective low energy, low momentum description, and we will see that these modes occur outside the regime of validity of the theory. However, as a 
practical method for studying the evolution of a heavy ion collision we are interested in a causal, stable set of differential equations that can be solved on a computer.

The problem with causality is related to the fact that in the Navier-Stokes equation the dissipative stresses are instantaneously equal to spatial gradients of the fluid velocity. In any microscopic treatment this is not the case - stresses are generated by strains in the flow velocity over some characteristic time. This effect automatically appears at second order in the gradient expansion, as we will see below. In a scale invariant fluid the most general form of the stress tensor is [158]

$$
\begin{aligned}
\delta T^{\mu \nu}=- & \eta \sigma^{\mu \nu}+\eta \tau_{R}\left[\left\langle D \sigma^{\mu \nu\rangle}+\frac{1}{3} \sigma^{\mu \nu}(\partial \cdot u)\right]\right. \\
& +\lambda_{1} \sigma^{\langle\mu}{ }_{\lambda} \sigma^{\nu\rangle \lambda}+\lambda_{2} \sigma_{\lambda}^{\langle\mu} \Omega^{\nu\rangle \lambda}+\lambda_{3} \Omega^{\langle\mu}{ }_{\lambda} \Omega^{\nu\rangle \lambda},
\end{aligned}
$$

where $\mathcal{O}^{\langle\mu \nu\rangle}=\frac{1}{2} \Delta^{\mu \alpha} \Delta^{\nu \beta}\left(\mathcal{O}_{\alpha \beta}+\mathcal{O}_{\beta \alpha}-\frac{2}{3} \Delta^{\mu \nu} \Delta^{\alpha \beta} \mathcal{O}_{\alpha \beta}\right)$ denotes the transverse traceless part of $\mathcal{O}^{\alpha \beta}$. The relativistic vorticity tensor is

$$
\Omega^{\mu \nu}=\frac{1}{2} \Delta^{\mu \alpha} \Delta^{\nu \beta}\left(\partial_{\alpha} u_{\beta}-\partial_{\beta} u_{\alpha}\right) .
$$

The quantities $\tau_{R}$ and $\lambda_{i}$ are second order transport coefficients. We note that Eq. 25 can also be obtained in kinetic theory [159, 160], but in this case certain terms allowed by the symmetries, like the $\lambda_{3}$-term in Eq. 25, are absent.

The coefficient $\tau_{R}$ describes the relaxation of the fluid stresses to the NavierStokes form. This can be seen more explicitly by writing the equations of fluid dynamics as

$$
\partial^{\mu}\left(T_{\mu \nu}^{(0)}+\pi_{\mu \nu}\right)=0
$$

where the viscous stresses $\pi_{\mu \nu}$ satisfy the dynamical equation

$$
\begin{aligned}
\tau_{R}^{\langle} D \pi^{\mu \nu\rangle}=- & \left(\pi^{\mu \nu}+\eta \sigma^{\mu \nu}\right)-\frac{4}{3} \tau_{R} \pi^{\mu \nu}(\partial \cdot u) \\
& +\frac{\lambda_{1}}{\eta^{2}} \pi_{\lambda}^{\langle\mu} \pi^{\nu\rangle \lambda}-\frac{\lambda_{2}}{\eta} \pi_{\lambda}^{\langle\mu} \Omega^{\nu\rangle \lambda}+\lambda_{3} \Omega_{\lambda}^{\langle\mu} \Omega^{\nu\rangle \lambda} .
\end{aligned}
$$

This equation is equivalent to Eq. 25 at second order in gradients of the fluid velocity. It has the form of a relaxation equation: The viscous stress $\pi^{\mu \nu}$ relaxes to the Navier-Stokes result $-\eta \sigma^{\mu \nu}$ on a time scale controlled by $\tau_{R}$.

The set of equations given by (27-28) provides a causal and stable set of fluid dynamic equations. In particular, the dispersion relation of the shear mode is given by

$$
\omega=\frac{i \nu k^{2}}{1+i \omega \tau_{R}},
$$


and the limiting speed is $v_{D}^{\max }=\left[\eta /\left(s \tau_{R} T\right)\right]^{1 / 2}$. We will see below that for reasonable values of $\tau_{R}$ we get $v_{D}^{\max }<c$, and the fluid dynamic equations are causal. Formally, the equations have second order accuracy in gradients, and the sensitivity to poorly constrained higher order transport coefficients can be checked by varying $\tau_{R}$ and $\lambda_{i}$.

\subsubsection{Nearly perfect fluidity and the convergence of the gradient expansion}

Another important issue is whether the gradient expansion converges in the case of relativistic heavy ion collisions. This is far from obvious, because the systems are small, initial energy density gradients are large, and the evolution is very rapid. We will provide more detailed estimates for real heavy ion collisions in Section 4.3. As a warm-up, we consider an equilibrium response function in a static medium. The shear stress response function is given by Eq. (11). In linear response theory this function controls the stress induced by an external strain. In fluid dynamics $T_{x y} \simeq(\mathcal{E}+P) u_{x} u_{y}$ and we can compute the correlation function from linearized hydrodynamics and fluctuation relations. We find $[152,161,162]$

$$
G_{R}^{x y x y}(\omega, k)=P-i \eta \omega+\tau_{R} \eta \omega^{2}+O\left(\omega^{3}, k^{2}\right) .
$$

This result, called the Kubo formula, can be used to relate the shear viscosity and other transport coefficients to an equilibrium (but time-dependent) correlation function in the microscopic theory, see Eq. (12). We can now read off the criterion that the gradient expansion converges. From the first two terms in Eq. (30) we get

$$
\omega \lesssim \frac{s T}{\eta}
$$

where we have used $P \sim s T$. Analogously, from the second and third term we get $\omega \lesssim \tau_{R}^{-1}$. This constraint is consistent with Eq. (31) provided $\tau_{R} \sim s T / \eta$, which is indeed what one finds both in the weak coupling, kinetic theory, and in the strong-coupling, AdS/CFT-like, regime [163, 158]. We note that this estimate of $\tau_{R}$ also implies that the limiting speed of a diffusive wave is the speed of light, consistent with causality.

In the early stages of a relativistic heavy ion collision the characteristic expansion time of the plasma, $\tau \sim(\partial \cdot u)^{-1}$, is significantly less than $1 \mathrm{fm}$. In order for the expansion parameter $\eta /(s T \tau)$ to be small, we need $\eta / s \ll 1$. Note that $R e^{-1}=\eta /(s T \tau)$ is the inverse Reynolds number of the flow. In kinetic theory one finds $[164,165]$

$$
\frac{\eta}{s} \simeq \frac{9.2}{g^{4} \log (1 / g)}
$$

where we have assumed that the quark-gluon plasma contains three light quark flavors. The argument inside the logarithm has been determined [166], but the 
expansion in inverse powers of $\log (1 / g)$ converges very slowly. For numerical estimates we will assume $\log (1 / g) \gtrsim 1$. Using $g \simeq 2$, which corresponds to $\alpha_{s} \simeq$ 0.3 , we conclude that at best $\eta / s \lesssim 0.6$. This estimate implies that fluid dynamics is not likely to be quantitatively reliable in relativistic heavy ion collisions.

This pessimistic view was revised because of two discoveries, one experimental and the other theoretical. The first is the discovery of nearly ideal flow, indicative of very small viscous corrections, observed in the early RHIC data $[167,168]$ and confirmed at the LHC [169]. The second is the theoretical realization that the strong coupling value of $\eta / s$ in gauge theories with holographic duals is as small as $\eta / s=1 /(4 \pi)[32,107]$. A similar lower bound on $\eta / s$ was first suggested based on the quantum mechanical uncertainty relation [170]. Note that, if we reinstate all physical constants, the proposed bound takes the form $\eta / s=\hbar /\left(4 \pi k_{B}\right)$, where $\hbar$ is Planck's constant, and $k_{B}$ is Boltzmann's constant.

We will refer to fluids that approach $\eta / s=1 /(4 \pi)$ as "nearly perfect fluids". Fluids of this type exhibit fluid dynamic behavior on time and distance scales as short as $t \sim l \sim T^{-1}$. The reason that hydrodynamics is successful as a theory of relativistic heavy ion collisions is that the quark-gluon plasma is a nearly perfect fluid. Indeed, as we shall demonstrate, the best determinations of $\eta / s$ at RHIC and the LHC are remarkably close to $1 /(4 \pi)$.

\subsubsection{Beyond gradients: Hydrodynamic fluctuations}

We have argued that fluid dynamics is a general effective theory that describes the long distance, late time response of a many body system perturbed away from thermal equilibrium. However, it is clear that in order to improve the fluid dynamic description it is not sufficient to include higher order gradients. Fluid dynamics is a coarse grained description, and as we attempt to increase the resolution local fluctuations in the fluid dynamic description become more important.

The equations of fluid dynamics including fluctuations can be written as $[171$, $172,173,174]$

$$
\partial^{\mu}\left(T_{\mu \nu}^{(0)}+\pi_{\mu \nu}+\Xi_{\mu \nu}\right)=0
$$

where $\Xi_{\mu \nu}$ is a stochastic term which satisfies

$$
\begin{aligned}
\left\langle\Xi_{\mu \nu}(x) \Xi_{\alpha \beta}\left(x^{\prime}\right)\right\rangle=[ & 2 \eta T\left(\Delta_{\mu \alpha} \Delta_{\nu \beta}+\Delta_{\mu \beta} \Delta_{\nu \alpha}\right) \\
& \left.-2\left(\zeta-\frac{2 \eta}{3}\right) T \Delta_{\mu \nu} \Delta_{\alpha \beta}\right] \delta\left(x-x^{\prime}\right) .
\end{aligned}
$$

The structure of Eq. (34) is fixed by fluctuation dissipation relations. As in Section 4.2.2 it is easiest to understand the role of these terms near equilibrium. 
We consider the response function for $T_{x y}$ and study the role of fluctuations $\delta T_{x y} \simeq(\mathcal{E}+P)_{0} u_{x} u_{y}$. The leading term involves a pair of velocity correlators, which can be viewed as a Feynman diagram with two propagators for sound or shear modes. For example, the propagator of a shear mode is given by

$$
\left\langle u_{x} u_{y}\right\rangle_{\omega k}=-\frac{2 T}{\mathcal{E}+P} \frac{k_{x} k_{y}}{k^{2}} \frac{\nu k^{2}}{\omega^{2}+\nu^{2} k^{4}} .
$$

In momentum space the convolution of the two propagators gives a loop integral. We find [175]

$$
\delta G_{R}^{x y x y}(\omega, 0)=-\frac{7 T \Lambda^{3}}{90 \pi^{2}}-i \omega \frac{17 T \Lambda}{120 \pi^{2} \nu}+(1+i) \omega^{3 / 2} T \frac{7+(3 / 2)^{3 / 2}}{240 \pi \nu^{3 / 2}}+O\left(\omega^{2}\right),
$$

where $\Lambda$ is a momentum space cutoff. Comparing this result with Eq. (30) reveals a number of very interesting features:

- Fluid dynamics behaves like a renormalizable effective theory. There are divergences, but the divergent terms can be absorbed into the low energy parameters, the pressure $P$ and the viscosity $\eta$. Non-analytic terms, like the $\omega^{3 / 2}$ term in Eq. (36) are finite.

- Fluid dynamics has an intrinsic resolution scale, given by the cell size in a simulation with fluctuating stresses, and the parameters of fluid dynamics, the equation of state and the transport coefficients, evolve as a function of that scale.

- Non-analytic corrections are smaller than the Navier-Stokes term - $\omega \eta$, but larger than the second order term $\omega^{2} \tau_{R} \eta$. This means that a consistent second order calculation has to contain fluctuating forces.

- The fluctuation contribution to the $i \omega$ term is inversely proportional to $\nu=$ $\eta /(s T)$. This suggests that the physical viscosity cannot become arbitrarily small [175]. The physical mechanism for this bound is related to the fact that shear viscosity is a measure of momentum diffusion, and that the contribution of shear and sound modes to momentum diffusion can never vanish. Note that this bound is completely classical - Planck's constant only enters indirectly, via the equation of state in the quantum regime [175].

In calculations of flow observables the contribution of thermal fluctuations is likely to be small compared to the role of initial state fluctuations (see Fig. 12) [172]. However, the formalism described here is important in describing the dynamical evolution of fluctuations discussed in Section 5, in particular in the vicinity of a critical point, where the rate at which fluctuations can grow becomes a crucial concern. 


\subsection{Collective expansion and transport properties of the quark-gluon plasma}

The experimental determination of transport properties of the quark-gluon plasma is mainly based on the comparison of flow measurements at collider energies $[176,177]$ with dissipative fluid dynamics simulations $[148,149]$. Several observations support the assumption that heavy ion collisions create a locally thermalized system:

- The total abundances of produced particles is described by a simple thermal model that depends on only two parameters, the temperature $T$ and the baryon chemical potential $\mu$ at freeze-out, see Section 3 .

- In the regime of transverse momenta $p_{\perp} \lesssim 2 \mathrm{GeV}$ the spectra $d N / d^{3} p$ of produced particles follow a modified Boltzmann distribution characterized by the freeze-out temperature and a collective radial expansion velocity $[19,148,178,179]$. Radial flow manifests itself in the fact that the spectra of heavy hadrons, which acquire a boost $p_{\perp} \sim m u_{\perp}$ from the collective radial expansion with velocity $u_{\perp}$, have a larger apparent temperature than the spectra of light hadrons.

- In non-central collisions the distribution of produced particles in the transverse plane shows a strong azimuthal anisotropy known as elliptic flow $[180,148,176]$. Elliptic flow represents the hydrodynamic response of the quark-gluon plasma to energy density gradients in the initial state. These gradients are caused by a combination of geometric effects, related to the overlap geometry, and fluctuation effects, related to the mechanism of the initial energy deposition.

The quantitative analysis of the transverse flow pattern is based on Fourier moments of the azimuthal particle distribution. We define the harmonic moments

$$
\begin{aligned}
\left.p_{0} \frac{d N}{d^{3} p}\right|_{p_{z}=0}=\left.p_{0} \frac{d N}{\pi d p_{T}^{2} d p_{z}}\right|_{p_{z}=0}(1 & 2 v_{1}\left(p_{T}\right) \cos \left(\phi-\Psi_{1}\right) \\
& \left.+2 v_{2}\left(p_{T}\right) \cos \left(2 \phi-\Psi_{2}\right)+\ldots\right),
\end{aligned}
$$

where $p_{z}$ is the momentum in the longitudinal (beam) direction, $p_{T}$ is the transverse momentum, $\phi$ is the transverse angle relative to the impact parameter direction. The coefficient $v_{2}$ is known as elliptic flow, and the higher moments are termed triangular, quadrupolar, etc. flow. The angles $\Psi_{i}$ account for the fact that the flow angles need not be aligned with the impact parameter plane, and are known as flow angles. Substantial elliptic flow, reaching about $v_{2}\left(p_{T}=\right.$ $2 \mathrm{GeV}) \simeq 20 \%$ in semi-central collisions, was observed in the early RHIC data $[167,168]$ and confirmed at the LHC [169]. More recently, it was realized that 
fluctuations in the initial energy density generate substantial higher harmonics, including odd Fourier moments such as $v_{3}$ [181], as well as fluctuations of the flow angles relative to the impact parameter plane [182].

Viscosity tends to equalize the radial flow velocity and suppress elliptic flow and higher flow harmonics. An estimate of the relevant scales can be obtained from simple scaling solutions of fluid dynamics. The simplest analytic solution was found by Bjorken, who considered a purely longitudinal expansion [183]. Bjorken assumed that the nuclei are infinitely extended in the transverse direction, and that the initial entropy density is independent of rapidity. This implies that the subsequent evolution is invariant under boosts along the $z$ axis. The Bjorken solution provides a natural starting point for more detailed numerical and analytical studies $[148,184]$. Bjorken flow is characterized by a flow profile of the form $u_{\mu}=\gamma\left(-1,0,0, v_{z}\right)=(-t / \tau, 0,0, z / \tau)$, where $\gamma=\left(1-v_{z}^{2}\right)^{1 / 2}$ is the boost factor and $\tau=\left(t^{2}-z^{2}\right)^{1 / 2}$ is the proper time. This velocity field solves the relativistic Navier-Stokes equation. Energy conservation then determines the evolution of the entropy density. We find

$$
-\frac{\tau}{s} \frac{d s}{d \tau}=1-\frac{4}{3} \frac{\eta}{s T \tau}
$$

where we have neglected bulk viscosity. Using both the ideal equation of state, $s \sim T^{3}$, and ideal hydrodynamic evolution we obtain $T \sim 1 / \tau^{1 / 3}$. The validity of the gradient expansion requires that viscous corrections in Eq. (38) are small $[170]$

$$
\frac{\eta}{s} \ll \frac{3}{4}(T \tau) .
$$

It is usually assumed that in the QGP $\eta / s$ is approximately constant. For the Bjorken solution $T \tau \sim \tau^{2 / 3}$ increases with time, and Eq. (39) is most restrictive during the initial stages of the expansion. Using an equilibration time $\tau_{0}=1 \mathrm{fm}$ and an initial temperature $T_{0}=300 \mathrm{MeV}$ gives $\eta / s \lesssim 0.6$. This confirms our earlier assertion that fluid dynamics can be applied to heavy ion collisions only if the QGP behaves as a nearly perfect fluid.

At late time the expansion becomes three dimensional and $T \tau$ is independent of time. The relevant degrees of freedom are hadronic resonances with interaction cross sections $\sigma$ that reflect hadronic sizes and are approximately independent of energy. In that case $\eta \sim T / \sigma$. Using $s \sim T^{3}$ and $T \sim 1 / \tau$ we find that the viscous correction $\eta /(s T \tau)$ increases with proper time as $\tau^{2}$. This result shows that fluid dynamics also breaks down at late times. At RHIC and LHC energies the duration of the fluid dynamic phase is $5-10 \mathrm{fm} / \mathrm{c}$, depending on collision energy and geometry.

In heavy ion collisions we do not directly observe the final distribution of energy and momentum. What is measured experimentally is the distribution 


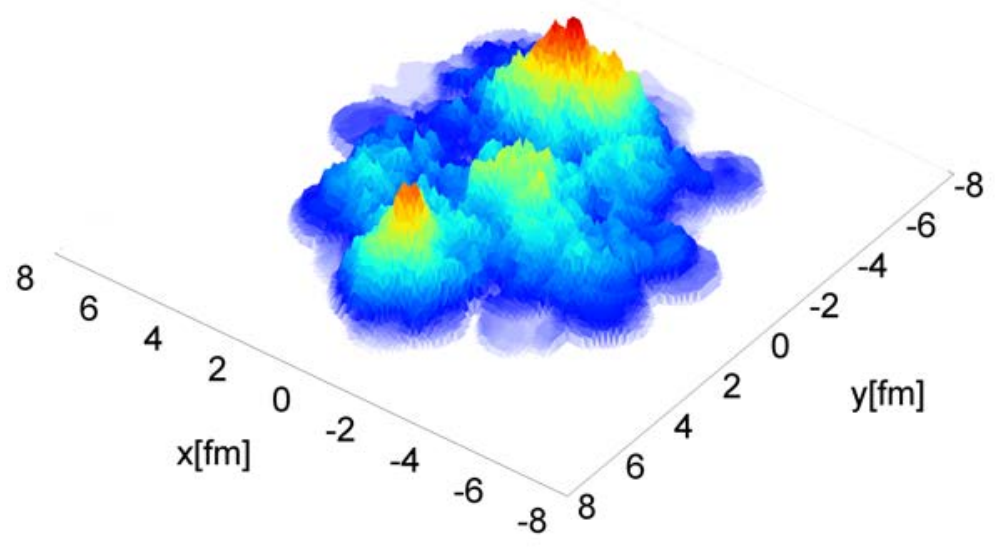

Figure 12: Initial energy density (arbitrary units) in a $\mathrm{Au}+\mathrm{Au}$ collision at RHIC from the Monte-Carlo KLN model, see $[185,186]$. This model include the effects from the collision geometry, fluctuations in the initial position of the nucleons inside the nucleus, and non-linear gluon field evolution. More sophisticated versions of the model also include quantum fluctuations of the gluon field.

of hadrons. In principle one could imagine reconstructing azimuthal harmonics of the stress tensor at freeze-out from the measured particle distribution, but doing so would require complete particle identification as well as spatial and momentum information for the produced particles, and it has not been attempted. In any case, hadrons continue to interact after the fluid freezes out, and some rearrangement of momentum takes place. This means that we need a prescription for converting hydrodynamic variables to kinetic distribution functions. What is usually done is that we define a freeze-out hypersurface on which hydrodynamics is assumed to break down. In principle, this hypersurface is defined by a kinetic criterion, for example the condition that the mean free path of a typical hadron satisfies $l_{m f p} \gtrsim c_{s} /(\partial \cdot u)$. In practice, freeze-out is assumed to take place at constant temperature or energy density. On the freeze-out surface the conserved densities in fluid dynamics are matched to kinetic theory [187].

In ideal fluid dynamics the distribution functions are Bose-Einstein or FermiDirac distributions characterized by the local temperature and fluid velocity. Viscosity modifies the stress tensor, and via matching to kinetic theory this modification changes the distribution functions $f_{p}$. The value of $\eta / s$ constrains only the $p_{i} v_{j}$ moment of the distribution function. The full distribution function can 
be reconstructed only if the collision term in the Boltzmann equation is specified. Using a simple model which assumes that collisions are fully specified by a single collision time (known as the BGK model [188]) one obtains a very simple formula for the leading correction $\delta f_{p}$

$$
\delta f_{p}=\frac{1}{2 T^{3}} \frac{\eta}{s} f_{0}\left(1 \pm f_{0}\right) p_{\alpha} p_{\beta} \sigma^{\alpha \beta},
$$

where the \pm sign refers to Bose/Fermi distributions. This result is a reasonable approximation to more microscopic theories [165]. The shift in the distribution function leads to a modification of the single particle spectrum. In the case of the Bjorken expansion and at large $p_{T}$ we find

$$
\frac{\delta(d N)}{d N_{0}}=\frac{1}{3 \tau_{f} T_{f}} \frac{\eta}{s}\left(\frac{p_{T}}{T_{f}}\right)^{2},
$$

where $d N_{0}$ is the number of particles emitted in ideal fluid dynamics, $\delta(d N)$ is the dissipative correction, and $\tau_{f}$ is the freeze-out time. Note that if it were not for nearly perfect fluidity, $\eta / s \simeq 1 /(4 \pi)$, the prediction of spectra using fluid dynamics at RHIC and LHC would be completely hopeless. Even with a minimal viscosity corrections are of order $25 \%$ at $p_{T} \sim 1 \mathrm{GeV}$. As a consequence, precision determinations of $\eta / s$ should be based on integrated observables that are dominated by low $p_{T} \lesssim 1 \mathrm{GeV}$.

In a system with strong longitudinal expansion viscous corrections tend to equalize the momentum distribution by pushing particles to higher $p_{T}$. Because the single particle distribution enters into the denominator of $v_{2}$ this effect suppresses $v_{2}$ at large $p_{T}$. The effect from the numerator, dissipative corrections related to the $\cos (2 \phi)$ component of the transverse flow, act in the same direction [189]. The important observation is that corrections to the spectrum are controlled by the same parameter $\eta /(s \tau T)$ that governs the derivative expansion in fluid dynamics. This reflects the fact that in the regime in which kinetic theory can be matched to fluid dynamics, the expansion parameter in kinetic theory, the Knudsen number

$$
K n=\frac{l_{m f p}}{L}
$$

with $L \sim c_{s} /(\partial \cdot u)$, is comparable to the expansion parameter in fluid dynamics, $K n \sim R e^{-1}[190]$.

We obtain several simple predictions that have been confirmed experimentally [177]: Dissipative corrections increase with $p_{T}$, they are larger in small systems that freeze out earlier, and they are larger for higher harmonics that are more sensitive to gradients of the radial flow profile. Quantitative studies that provide reliable measurements of $\eta / s$ together with estimates of the uncertainties involved require a number of ingredients [193]: 


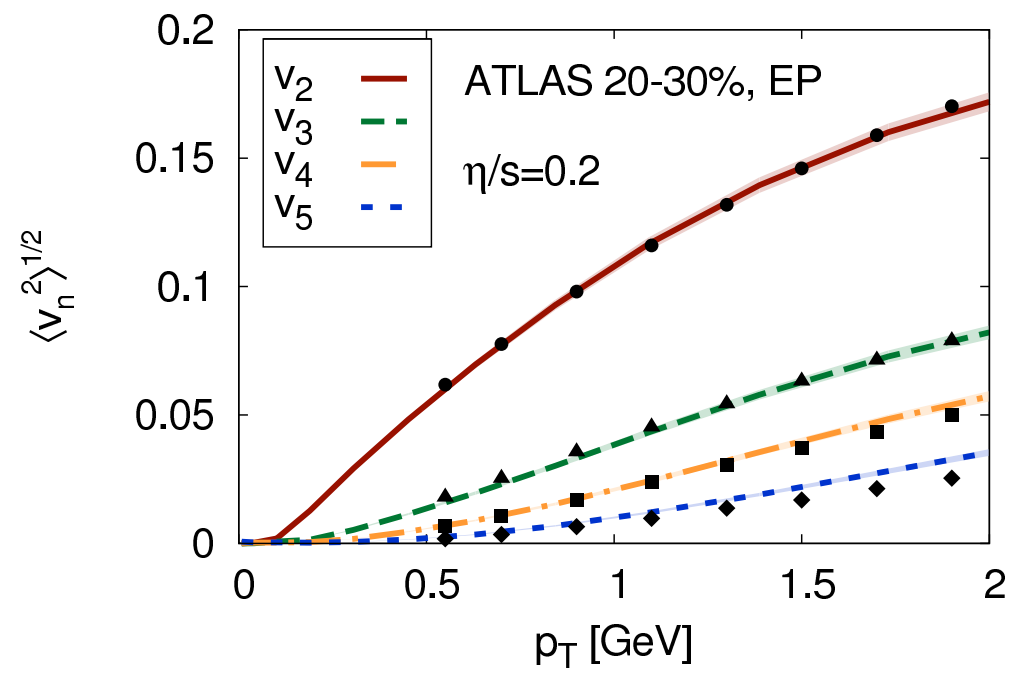

Figure 13: Fourier coefficients $v_{2}, \ldots, v_{5}$ of the azimuthal charged particle distribution as a function of the transverse momentum $p_{T}$ measured in $P b+P b$ collisions at the LHC [191] The lines show a hydrodynamic analysis performed using $\eta / s=0.2$ [192].

- An initial state model that incorporates the nuclear geometry and fluctuations in the initial energy deposition. The simplest possibility is a MonteCarlo implementation of the Glauber model [194], but some calculations also include a model of the color field generated by colliding nuclei, as well as the effects of quantum fluctuations and real time evolution of this field [192]. Alternatively, one may try to describe the pre-equilibrium stage using kinetic theory $[195,196]$ or the AdS/CFT correspondence [197]. At the end of the initial stage the stress tensor is matched to fluid dynamics.

- Second order dissipative fluid dynamics in $2+1$ (boost invariant) or $3+1$ dimensions with a realistic equation of state (EOS). A realistic EOS has to match lattice QCD results at high temperature, and a hadronic resonance gas below $T_{c}$ [198]. The resonance gas EOS must allow for chemical nonequilibrium effects below the chemical freeze-out temperature $T_{\text {chem }} \simeq T_{c}$. It is important to check the sensitivity to poorly constrained second order transport coefficients.

- Kinetic freeze-out and a kinetic afterburner. At the kinetic freeze-out temperature the fluid is converted to hadronic distribution functions. Ideally, these distribution functions are evolved further using a hadronic transport model [199, 200], but at a minimum one has to include feed-down from hadronic resonance decays. 


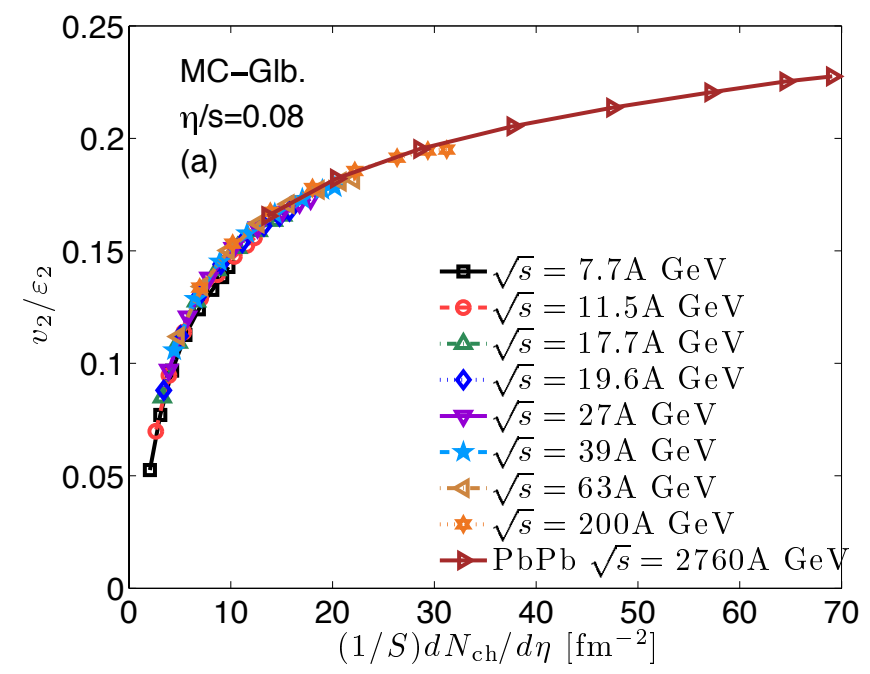

Figure 14: Eccentricity scaled elliptic flow $v_{2}$ plotted as a function of the charged hadron multiplicity density $d N / d y$ divided by the nuclear overlap area $S$ for different collision energies, from [209]. The figure shows results from a hydrodynamic simulation with MC-Glauber initial conditions and $\eta / s=0.08$.

The first constraints on $\eta / s$ based on the RHIC data were derived in [189], and the effects of dissipation in the hadronic corona were studied in [201, 202, 200]. Early estimates of $\eta / s$ were also obtained in [203] using the relationship between heavy quark diffusion and momentum diffusion in the plasma obtained in kinetic theory [204]. Determinations of $\eta / s$ at RHIC based on viscous fluid dynamics were obtained in $[33,34,205]$. A more recent analysis of LHC data is shown in Fig. 13 [192]. The authors found $\eta / s \simeq 0.2$ at the LHC, and $\eta / s \simeq 0.12$ from a similar analysis of RHIC data. Similar results were obtained by other authors. Song et al. reported an average value of $\eta / s \simeq(0.2-0.24)$ at the LHC and $\eta / s \simeq 0.16$ at RHIC [206]. Luzum and Ollitrault tried to constrain the allowed range of $\eta / s$, obtaining $0.07 \leq \eta / s \leq 0.43$ at RHIC [207]. Given the complexity of the analysis, uncertainties are difficult to quantify. A survey of the main sources of error in the determination of $\eta / s$ can be found in [208]. Interestingly, the extracted values of $\eta / s$ are lower at RHIC than they are at the LHC, as one would expect based on asymptotic freedom.

\subsection{Frontiers: Flow in $p A$ ?}

An important recent discovery is the observation of significant elliptic and triangular flow in high multiplicity $\mathrm{p}+\mathrm{Pb}$ collisions at the LHC $[210,211,212]$. A particularly striking discovery is the mass ordering of $v_{2}\left(p_{T}\right)$ [212], which is usually regarded as strong evidence for collective expansion [148]. The result is 


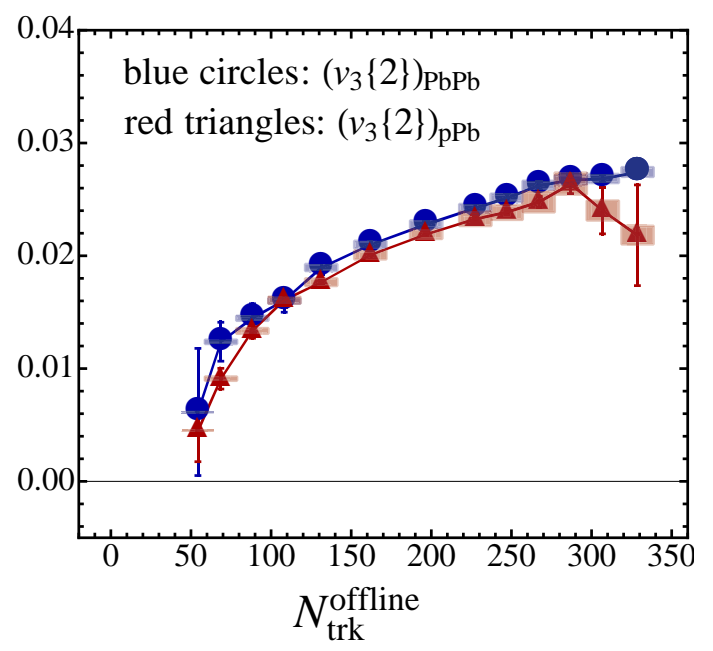

Figure 15: Integrated $v_{3}\{2\}$ for $P b P b$ and $p P b$ at the LHC as a function of the multiplicity. Here, $v_{3}\{2\}$ denotes the triangular flow extracted from two-particle cumulants. Data from [210], analysis taken from [216].

surprising, because the proton nucleus collisions have generally been regarded as a control experiment in which dissipative corrections are too large for collective flow to develop. Indeed, several authors have shown that initial state effects [213, 214], or a simple free-streaming expansion [215], can give significant contributions to flow observables in small systems.

We should note, however, that the observed response to initial state fluctuations in nucleus-nucleus collisions indicates that the mean free path is very short, comparable to size of a single nucleon. The measured flow in $p A$ may well be due to the same mechanism that generates flow in $A A$, the hydrodynamic response to initial energy density gradients. Ultimately, only detailed studies of many particle correlations along the lines of [41] can disentangle the relative importance of collective and non-collective effects.

In this review we will concentrate on a simpler question, whether there are simple scaling variables that can be used to compare experimental data from collisions at different beam energies, impact parameters, and nuclear mass numbers. An important step in this direction was taken by Heiselberg and Levy, who studied elliptic flow in the dilute limit [217]. They showed that the contribution from single elastic scattering events is

$$
\frac{v_{2}}{\epsilon_{2}} \sim \frac{1}{S} \frac{d N}{d y}\langle\sigma\rangle .
$$

where $S$ is the transverse overlap area, $d N / d y$ is the multiplicity per unit of 
rapidity, and $\epsilon_{2}$ is the initial deformation, defined by

$$
\epsilon_{2}=\frac{\left\langle y^{2}-x^{2}\right\rangle}{\left\langle y^{2}+x^{2}\right\rangle} .
$$

Following the arguments given in the previous Section we expect that the parameter $(1 / S)(d N / d y)\langle\sigma\rangle$ also appears in fluid dynamics. This is indeed the case, as we can see using the following argument [218]. Consider a fireball of transverse size $\bar{R} \simeq \sqrt{R_{x}^{2}+R_{y}^{2}}$ which is undergoing Bjorken expansion in the longitudinal direction. The time scale for transverse expansion is $\tau=\bar{R} / c_{s}$, and the density at this time is $n \sim(\tau S)^{-1}(d N / d y)$. This implies that the inverse Knudsen number is

$$
\frac{1}{K n}=\frac{\bar{R}}{l_{m f p}}=\bar{R} n\langle\sigma\rangle=c_{s} \frac{1}{S} \frac{d N}{d y}\langle\sigma\rangle
$$

Knudsen number scaling of $v_{2} / \epsilon_{2}$ was first studied by Voloshin and Poskanzer, see $[219,220]$. The results compiled in [220] demonstrate nice data collapse if different systems, centralities, and beam energies are plotted as a function of $(1 / S)(d N / d y)$. The compilation also shows that $v_{2} / \epsilon_{2}$ rises almost linearly with $(1 / S)(d N / d y)$, and that the RHIC data at $200 \mathrm{GeV}$ per nucleon saturate the flow predicted by ideal hydrodynamics. A more recent analysis of data from the RHIC beam energy scan and $P b+P b$ collisions at the LHC is shown in Fig. 14 [209]. There is some uncertainty related to different models for $\epsilon_{2}$. Here, we show results based on the Monte Carlo Glauber model. In this case data collapse is excellent, but the results for MC-KLN model are not quite as good [209]. We observe some curvature in $v_{2} / \epsilon_{2}$. This means that there are viscous effects even at LHC energy, and that there is no saturation of flow.

An important assumption in Fig. 14 is that the effective cross section is not a function of the collision parameters. At high temperature the quark-gluon plasma is scale invariant and we expect $\langle\sigma\rangle \sim s^{-2 / 3}$. Then

$$
\frac{1}{K n} \sim\left(c_{s} \frac{d N}{d y}\right)^{1 / 3},
$$

and the overlap area does not appear in the estimate for the Knudsen number. The choice of scaling variable makes a significant difference when comparing $p A$ and $A A$ collisions as shown in [216]. Fig. 15 shows the $p_{T}$ integrated triangular flow in $p P b$ and $P b P b$ collisions at the LHC, plotted as a function of the multiplicity. We observe excellent data overlap, suggesting that a common mechanism is at work, and that the conformal Knudsen scaling holds at the highest energies. 


\subsection{Other frontiers, and puzzles}

There are several other frontiers, and some puzzles, that are worth mentioning:

- Flow even in $p p$ ? Azimuthal two-particle correlations very similar to those produced by elliptic and triangular flow in $A A$ collisions have also been observed in very high multiplicity $p p$ collisions at $\sqrt{s}=7 \mathrm{TeV}[221]$ and $\sqrt{s}=13 \mathrm{TeV}$ [222]. These observations are intriguing, but it has not been checked whether these are true multi-particle correlation, as was done in the case of $p P b$ collisions [41].

- Large photon elliptic flow: The photon $v_{2}\left(p_{T}\right)$ has been measured at RHIC and LHC [223, 224], and the result is comparable (within sizable errors) to the elliptic flow of light hadrons. This is surprising, because photon emission is expected to be dominated by the early stages of the quark-gluon plasma evolution before a significant collective flow can develop [225]. The discrepancy between theory and experiment is smaller at the LHC compared to RHIC, and it is further reduced by significant emission in the hadronic phase [226].

- Approximate beam energy independence of the charged particle elliptic flow $v_{2}\left(p_{T}\right)$ : The elliptic flow coefficient of charged particles has been measured over a large range of beam energies, from the low end of the beam energy scan at RHIC, $\sqrt{s_{N N}}=7.7 \mathrm{GeV}$, to the initial LHC energy $\sqrt{s_{N N}}=2.76$ $\mathrm{TeV}[169,227,228]$. For a given centrality the results are essentially beam energy independent. Within hydrodynamics this is somewhat surprising because many parameters, such as the lifetime of the system and $\eta / s$ are definitely changing. The observed universality could be accidental, because both the $v_{2}$ of identified particles, and the $p_{T}$ integrated $v_{2}$ do show beam energy dependence.

- Anomalous hydrodynamics: Several novel fluid dynamic effects have been discovered in recent years. An example is the chiral magnetic effect. Topological charge fluctuations in the initial state of a heavy ion collision, combined with the magnetic field generated by the highly charged ions, can manifest themselves in electric charge fluctuations in the final state [229]. This effect is now understood as part of a broader class of anomalous hydrodynamic effects [230]. An interesting recent observation is a measurement of charge dependent elliptic flow at RHIC [231], which could be interpreted as a manifestation of a new hydrodynamic mode, a chiral magnetic wave [232]. 


\section{Correlations and Fluctuations}

Fluctuations and correlations are important characteristics of any physical system. They provide essential information about the effective degrees of freedom and their possible quasi-particle nature.

In general, one can distinguish between several types of fluctuations. On the most fundamental level there are quantum fluctuations, which arise if we measure several non-commuting observables, or an observable that does not commute with the Hamiltonian. In a system that thermalizes we encounter thermal fluctuations. These reflect the fact that thermodynamics and hydrodynamics are coarse-grained descriptions, and that thermodynamic variables necessarily fluctuate in a finite sub-volume. An example is given by density fluctuations, which are controlled by the compressibility of the system. Finally, the dynamical evolution of a system may amplify small quantum or thermal fluctuations in the initial state.

In heavy-ion collisions, we encounter fluctuations and correlations related to the initial state of the system, fluctuations reflecting the subsequent evolution of the systems, and trivial fluctuations induced by the experimental measurement process. Initial state fluctuations are inhomgeneities in the initial energy and baryon number deposition, see Fig. 12. These fluctuations are quite substantial, and are reflected in higher harmonics of the radial flow field. If the systems thermalizes and is described by fluid dynamics then we expect that fluctuations in the subsequent evolution are mostly thermal. Thermal fluctuations are typically small, suppressed by $1 / \sqrt{N}$ where $N$ is the average number of particles in the volume considered. However, thermal fluctuations can become large in the vicinity of a second order phase transition. This is the phenomenon of critical opalescence. Finally, fluctuations related to the detectors need to be understood, controlled and subtracted in order to access the dynamical fluctuations which tell as about the properties of the system.

A well known example for fluctuations in a physical system are those of the cosmic microwave background first seen by the COBE satellite [233] and later refined by WMAP [234] and, most recently, by the Planck satellite [235]. In case of the cosmic microwave background the observed fluctuations are at the level of $10^{-4}$ with respect to the thermal background. In addition a large dipole correlation due to the motion of earth through the heat bath of the microwave background is observed. In heavy ion collision we are faced with a qualitatively similar situation. To leading order the observed particles follow a thermal distribution embedded in a Hubble-like radial flow field. In addition, for non-central collisions one observes a quadrupole correlation due to elliptic flow.

Experimentally fluctuations are most effectively studied by measuring socalled event-by-event (E-by-E) fluctuations, where a given observable is measured on an event-by-event basis and its fluctuations are studied for the ensemble of 
events. Alternatively, one may analyze the appropriate multi-particle correlations measured over the same region in phase space [236].

\subsection{Fluctuations in a thermal system}

As discussed in Section 3 there is good evidence that the system created in a ultrarelativistic heavy ion collision is, to a very good approximation, in thermal equilibrium. Therefore, let us start our discussion with thermal fluctuations. These are characterized by the appropriate cumulants of the partition function or, equivalently, by equal-time correlation functions which in turn correspond to the space-like (static) responses of the system.

In the following we will concentrate on fluctuations or cumulants of conserved charges, such as baryon number and electric charge. Therefore, we will work within the grand-canonical ensemble, where the system is in contact with an energy and "charge" reservoir. Consequently, the energy and the various charges are only conserved on the average with their mean values being controlled by the temperature and the various chemical potentials. As far as heavy ion reactions are concerned, the grand canonical ensemble appears to be a good choice as long as one only considers a sufficiently small subsystem of the entire final state, and, as discussed in Section 3, the final state hadron yields are very well described by a grand canonical thermal system of hadrons.

Fluctuations of conserved charges are characterized by the cumulants or susceptibilities of that charge. Given the partition function of the system with conserved charges $Q_{i}$

$$
Z=\operatorname{Tr}\left[\exp \left(-\frac{H-\sum_{i} \mu_{i} Q_{i}}{T}\right)\right]
$$

the susceptibilities are defined as the derivatives with respect to the appropriate chemical potentials. In case of three flavor QCD the conserved charges are the baryon number, strangeness and electric charge, $(B, S, Q)$, and we have

$$
\chi_{n_{B}, n_{S}, n_{Q}}^{B, S, Q} \equiv \frac{1}{V T^{3}} \frac{\partial^{n_{B}}}{\partial\left(\mu_{B} / T\right)^{n_{B}}} \frac{\partial^{n_{S}}}{\partial\left(\mu_{j} / T\right)^{n_{S}}} \frac{\partial^{n_{Q}}}{\partial\left(\mu_{Q} / T\right)^{n_{Q}}} \ln Z .
$$

The above susceptibilities ${ }^{2}$ may also be expressed in terms of of derivatives of the pressure $P=T / V \ln (Z)$

$$
\chi_{n_{B}, n_{S}, n_{Q}}^{B, S, Q}=\frac{\partial^{n_{B}}}{\partial\left(\mu_{B} / T\right)^{n_{B}}} \frac{\partial^{n_{S}}}{\partial\left(\mu_{j} / T\right)^{n_{S}}} \frac{\partial^{n_{Q}}}{\partial\left(\mu_{Q} / T\right)^{n_{Q}}}\left(\frac{P}{T^{4}}\right)
$$

\footnotetext{
${ }^{2}$ Here we adopt the normalization commonly used in the lattice QCD literature which differs from other normalization e.g. in [237].
} 
Consequently, these susceptibilities also control the pressure at small values of the various chemical potentials. For example, at small baryon number chemical potential, $\mu_{b} / T<1$, the pressure may be expressed in terms of a Taylor series

$$
\frac{P\left(T, \mu_{B}\right)}{T^{4}}=\frac{P\left(T, \mu_{B}=0\right)}{T^{4}}+\sum_{n} c_{n}(\mu / T)^{n}
$$

where the expansion coefficients are given by the baryon-number susceptibilities

$$
c_{n}=\frac{\chi_{n}^{B}}{n !}
$$

Due to the fermion sign problem, at present lattice QCD calculations can only be reliably carried out at vanishing chemical potentials. Therefore, the above Taylor expansion for the pressure is employed in order to determine the QCD equation of state for small chemical potentials [62, 65, 238]. Meanwhile many susceptibilities at various orders and various combinations of conserved charges have been calculated in lattice QCD. In the following we will discuss a selection of these results and their interpretation also in the context of experiment.

\subsubsection{Example: Net Charge Fluctuations}

To illustrate how fluctuations may be utilized to explore the relevant degrees of freedom, let us briefly discuss the fluctuations of the electric charge. In Refs. $[239,240]$ it has been realized that the electric charge of particles contributes in square to the fluctuations of the net-charge. Therefore, cumulants of the netcharge are in principle sensitive to the fractional charge of quarks in a quark-gluon plasma. This can be easily seen by considering the variance of the net charge of a gas of uncorrelated particles with charge $q$,

$$
\left\langle(\delta Q)^{2}\right\rangle=q^{2}\left\langle(\delta N)^{2}\right\rangle=q^{2}\langle N\rangle
$$

where in the last step we have, for simplicity, assumed that the particle number follows a Poisson distribution. Since the variance depends not only on the squared charge of the particles but also on the number of particles, it is advantageous to scale the charge variance by another extensive quantity, such as the entropy, $S$, so that the ratio

$$
R=\frac{\left\langle(\delta Q)^{2}\right\rangle}{S}
$$

does not depend on the size of the system. A simple estimate using Boltzmann statistics gives [239, 241]

$$
R_{Q G P}=\frac{1}{24}
$$




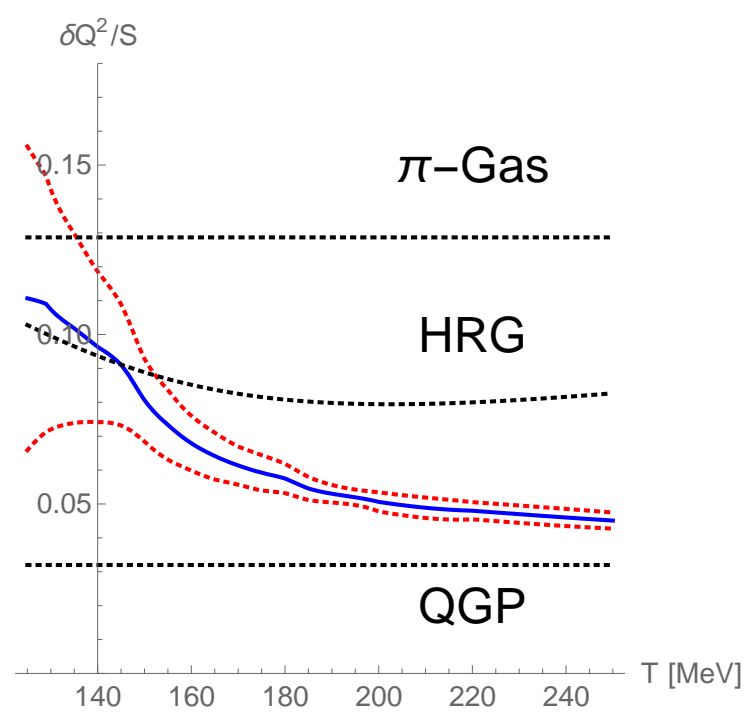

Figure 16: Net-charge variance per entropy, $R$, as a function of temperature from $2+1$ flavor lattice QCD with physical quark masses. The red-dashed line indicate the uncertainty. Results for $\left\langle(\delta Q)^{2}\right\rangle$ are from [244] and the entropy is extracted from [85]. The dashed horizontal lines indicate the results for a massless pion gas, a hadron gas as well as a non-interacting QGP with three flavors of massless quarks.

for a two flavor quark-gluon plasma whereas for a gas of massless pions we get

$$
R_{\pi}=\frac{1}{6}
$$

In other words, due to the fractional charges of the quarks, the charge fluctuations per entropy in a QGP is roughly a factor four smaller than that in a pion gas at the same temperature. In reality the hadronic phase is made out of more than pions, and, taking into account hadronic resonances, the charge variance per entropy is reduced by about $30 \%$ which still leaves roughly a factor three difference between a hadronic system and a QGP. Incidentally, the fact that charges contribute in square to fluctuations has been utilized to identify the fractional charges in a quantum Hall system as well as the double charge of cooper pairs in measurements of shot noise [242, 243].

While our simple example is instructive, in reality one has to include strange quarks and hadrons, quantum statistics, and possible correlations among quarks or hadrons. Therefore, a realistic calculation of $R$ will require lattice QCD methods. Since both the variance of the net charge and the entropy are well defined thermodynamic quantities this can be done, and in Fig. 16 we show the lattice QCD result for the net-charge variance per entropy based on the results for the 
net-charge variance from [244] and for the entropy density from [85]. We also show the results for a free pion gas and a QGP with three flavors of mass-less quarks, both using the proper quantum statistics, as well as that for a hadron resonance gas. We see that the hadron resonance gas agrees well with the lattice results for temperatures up to $T \lesssim 160 \mathrm{MeV}$, which is close to the pseudocritical temperature of $T_{p c}=154 \pm 9 \mathrm{MeV}$. For temperatures in the range of $160 \mathrm{MeV} \lesssim T \lesssim 250 \mathrm{MeV}$ the lattice calculations are in between the prediction a resonance gas and that of a non-interacting QGP, indicating that some of the correlations leading to resonance formation are still present in the system. With few exceptions, this trend is seen for most quantities which have been calculated on the lattice, such as energy density, cumulant ratios etc.: Good agreement with the hadron resonance gas up to the critical temperature, followed by a rather smooth transition to a free QGP which takes place over a temperature interval of approximately $\Delta T \sim 100 \mathrm{MeV}$, where the correlations slowly disappear.

\subsubsection{Correlations and mixed flavor susceptibilities}

Some of these correlations, namely those between the various flavors, can be explored explicitly by studying so called mixed flavor or "off-diagonal" cumulants. One example is the co-variance between strangeness and baryon number, $\langle\delta B \delta S\rangle \sim \chi_{1,1}^{B, S}$. Here $S$ refers to strangeness and not, as in the previous section, to the entropy. To illustrate the sensitivity of this co-variance to correlations among quarks, let us again compare a non-interacting QGP with a non-interacting hadron resonance gas (HRG). In the QGP strangeness is carried exclusively by baryons, namely the strange quarks, whereas in a HRG strangeness can also reside in strange mesons. Therefore, baryon number and strangeness are more strongly correlated in a QGP than in a hadron gas, at least at low baryon number chemical potential, where the mesons dominate. To quantify this observation, Ref. [245] proposed the following quantity

$$
C_{B S} \equiv-3 \frac{\langle\delta B \delta S\rangle}{\left\langle\delta S^{2}\right\rangle}=1+\frac{\langle\delta u \delta s\rangle+\langle\delta d \delta s\rangle}{\left\langle\delta s^{2}\right\rangle}
$$

where we have expressed $C_{B S}$ also in terms of quark degrees of freedom, noting that the baryon number of a quark is $1 / 3$ and the strangeness of a s-quark is negative one. Here $(u, d, s)$ represent the net-number of up, down and strange quarks, i.e. the difference between up and anti-up quarks etc. For a non-interacting QGP, $\langle\delta u \delta s\rangle=\langle\delta d \delta s\rangle=0$, so that $C_{B S}=1$. For a gas of kaons and anti-kaons, on the other hand, where a light (up or down) quark is always correlated with a strange anti-quark (kaons) or vice versa (anti-kaons) $\langle\delta u \delta s\rangle<0$, resulting in $C_{B S}<1$. Strange baryons, on the other hand, correlate light quarks with strange quarks or light anti-quarks with strange anti-quarks, so that $\langle\delta u \delta s\rangle>0$. Therefore, for sufficiently large values of the baryon number chemical potential, $C_{B S}>1$ for 


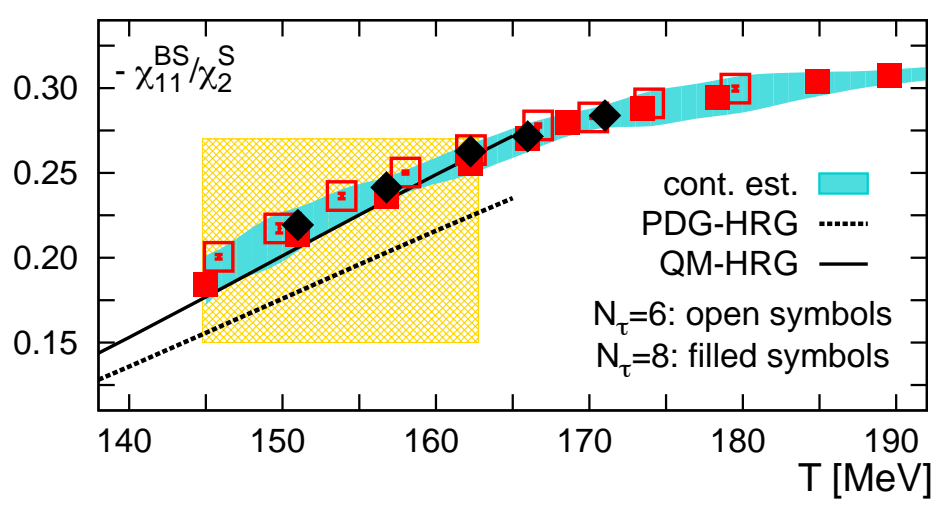

Figure 17: Lattice QCD results for $-\frac{\Xi_{B S}^{11}}{\Xi_{S}^{2}}=\frac{1}{3} C_{B S}$ together with results from hadron resonance gas with (full line) and without (dashed line) extra strange mesons. Figure adapted from [144].

a hadron gas, whereas for a non-interacting QGP $C_{B S}=1$ for all values of the chemical potential [245]. Since $C_{B S}$ can be expressed in terms of susceptibilities, $C_{B S}=-3 \frac{\chi_{B S}^{11}}{\chi_{S}^{2}}$, it can and has been calculated on the lattice with physical quark masses by two groups $[244,246]$. Both calculations agree with each other, and both report a small, but significant difference between the lattice results and that from the hadron resonance gas. In [144] it has been argued that this discrepancy may be removed by allowing for additional strange hadrons, which are not in the tables of the Particle Data Group (PDG) [247], but are predicted by various quark models. This is shown in Fig. 17, where the lattice QCD results are compared with a hadron resonance gas based on all the hadrons in the Review of Particles [247] (dotted line) and a hadron gas with additional strange hadrons (full line). Whether or not this turns out to be the correct explanation, this comparison demonstrates that these cumulant ratios are a sensitive probe of the relevant microscopic degrees of freedom.

To further explore at what temperature a hadronic description fails, one can study even more involved combinations of cumulant ratios to project out baryonic or mesonic states [144, 145, 248]. For example, in a hadron resonance gas, which is well described in the Boltzmann approximation, the pressure may be written as

$$
\begin{aligned}
P / T^{4} & =\sum_{\text {Baryons } i} \cosh \left(\hat{\mu}_{B} B_{i}+\hat{\mu}_{S} S_{i}+\hat{\mu}_{Q} Q_{i}\right) f\left(g_{i}, m_{i}, T\right) \\
& +\sum_{\text {Mesons } j} \cosh \left(\hat{\mu}_{S} S_{j}+\hat{\mu}_{Q} Q_{j}\right) f\left(g_{j}, m_{j}, T\right),
\end{aligned}
$$




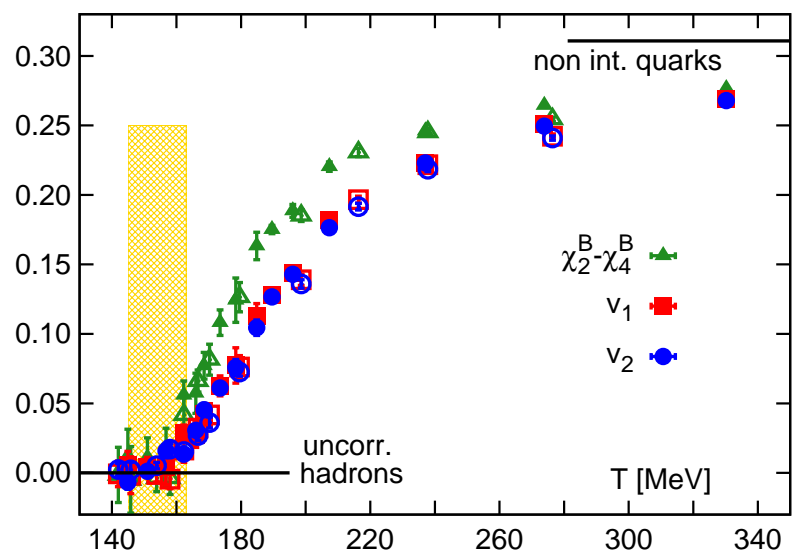

Figure 18: Various combinations of higher order cumulants which demonstrate the melting of the hadrons. See text for details. Figure adapted from [248].

where $\hat{\mu}_{B, S, Q}=\frac{\mu_{B, s, Q}}{T}$ and $f\left(g_{i}, m_{i}, T\right)=\frac{g_{i}}{2 \pi^{2}} \frac{m^{2}}{T^{2}} K_{2}(m / T)$. Since the baryon number of all baryons in the hadron gas is \pm 1 , the difference

$$
\chi_{2}^{B}-\chi_{4}^{B}=0
$$

vanishes in a hadron gas. For a non-interacting QGP, on the other hand, all quarks carry baryon number $B_{\text {quark }}= \pm 1 / 3$, and the difference between second and fourth order baryon-number cumulant is finite,

$$
\chi_{2}^{B}-\chi_{4}^{B}=\left(\frac{1}{9}-\frac{1}{81}\right) P_{\text {quarks }} / T^{4}>0 .
$$

The same holds for other, more complicated, combinations, involving strange particles, such as [248]

$$
\begin{aligned}
& v_{1}=\chi_{31}^{B S}-\chi_{11}^{B S} \\
& v_{2}=\frac{1}{3}\left(\chi_{2}^{S}-\chi_{4}^{S}\right)-2 \chi_{13}^{B S}-4 \chi_{22}^{B S}-2 \chi_{31}^{B S}
\end{aligned}
$$

Here, $v_{1}$ and $v_{2}$ represent combinations of cumulants, and, thus, should not be confused with the moments of the azimuthal distribution discussed in previous sections, which are commonly denoted by $v_{n}$ as well. In Fig. 18 we show the results from lattice QCD for these various combinations of cumulants as a function of temperature. All start deviating from the HRG value of zero at about the same temperature, indicating that both light and strange hadrons seems to loose their identity at temperatures above $T \gtrsim 150 \mathrm{MeV}$, which coincides with the 
pseudo-critical temperature of the QCD transition. A similar exercise has also been carried out for charmed hadrons [145] with the somewhat surprising result that even hadrons with open charm seem to "melt" at the same temperature of $T \sim 150 \mathrm{MeV}$.

To summarize this Section, we have demonstrated that the cumulants of conserved charges contain useful information about the correlations and relevant degrees of freedom of QCD matter. Since they are amenable to lattice QCD methods, the insights derived from such studies are rather model independent.

\subsection{Non-equilibrium and Experimental considerations}

Given the wealth of information which can be extracted from cumulants of conserved charges and the fact that they can be determined model independently, it would be very desirable to measure these cumulants in heavy ion collisions. However, a heavy ion collision is a highly dynamical process whereas lattice QCD deals with a static system in global equilibrium. In addition, real experiments have limitations in acceptance etc, which are difficult to map onto a lattice QCD calculation. Consequently a direct comparison of experiment with lattice QCD results for fluctuation observables is a non-trivial task. In the following we will discuss various issues which need to be understood and addressed in order for such a comparison to be meaningful.

- Dynamical evolution: So far our discussion assumed that the system is static and in global thermal equilibrium. However, even if fluid dynamics is applicable the system is at best in local thermal equilibrium, and in viscous fluid dynamics local equilibrium is never complete. The difference between local and global thermal equilibrium is an important aspect of the evolution of fluctuations of conserved charges, because the amount of conserved charge in a given comoving volume can only change by diffusion, and the rate of diffusion is limited by causality [249]. This observation is central to the use of the variable $R$ defined in Eq. (53) to detect the presence of quark-gluon plasma. If we consider a sufficiently large rapidity window $\Delta y$ then the value of $R$ is frozen in during the QGP phase, and cannot change in the subsequent hadronic stage. Of course, if $\Delta y$ is chosen too large, then $R$ never equilibrates, and reflects properties of the initial state.

This observation can be made more quantitative using the theory of fluctuating hydrodynamics outlined in Section 4.2.3. However, so far most theoretical studies have focused on schematic models, see, for example [250]. More importantly, there are other physical and experimental considerations that affect the choice of $\Delta y$, which we will discuss next.

- Global charge conservation: Obviously, baryon number, electric charge and strangeness are conserved globally, i.e. if we detected all particles, none 
of the conserved charges would fluctuate. In contrast, lattice QCD calculations are carried out in the grand canonical ensemble, which allows for exchange of conserved charges with the heat bath. Consequently, charges are conserved only on the average and, thus, do fluctuate due to the exchange with the heat-bath. These exchanges and thus the fluctuations depend on the correlations between particles and, as demonstrated above, on the magnitude of the charges of the individual particles. Therefore, in order to compare with lattice QCD, one has to mimic a grand canonical ensemble in experiment. This can be done by analyzing only a subset of the particles in the final state. However, even in this case, corrections due to global charge conservation are present. These corrections increase with the order of the cumulant [251] and need to be taken into account as discussed in $[250,252,253,254]$.

- Finite acceptance: All real experiments do have a finite acceptance, i.e. they are not able to cover all of phase space. In addition, most experiments are unable to detect neutrons, which do carry baryon number. However, due to rapid isospin exchange processes, the lack of neutron detection may be successfully modeled by a binomial distribution [254]. While it is desirable to study only a subset of particles, in order to mimic a grand canonical ensemble, it is mandatory to have sufficient coverage in phase space in order to capture all correlations. We note, that at the lowest beam energy, $\sqrt{s}=7.7 \mathrm{GeV}$, STAR finds a rather significant dependence on the width of the rapidity window for the fourth order net-proton cumulant [255].

- Efficiency corrections: A real world experiment detects a given particle only with a probability, commonly referred to as efficiency $\epsilon$, which is smaller than one, $\epsilon<1$. However, this does not mean that in every event one detects the same fraction of produced particles. Consequently, the number of measured particles fluctuates even if the number of produced particles does not. In other words the finite detection efficiency gives rise to fluctuations, which need to removed or unfolded before a comparison with any theoretical calculation. If the efficiency follows a binomial distribution, analytic formulas for the relation between measured and true cumulants can be derived $[256,257,258]$. These have been applied to the most recent analysis by the STAR collaboration.

- Dynamical fluctuations: A heavy ion collision is a highly dynamical process and the initial conditions as well as the time evolution may easily give rise to additional fluctuations. Especially at lower energies, $\sqrt{s} \lesssim$ $30 \mathrm{GeV}$, the incoming nuclei are stopped effectively and deposit baryon number and electric charge in the mid-rapidity region. Clearly the amount 
of baryon number deposited will vary from event to event, resulting in fluctuations of the baryon number at mid-rapidity, which are not necessarily the same as those of a thermal system. This potential source of background needs to be understood and removed, especially at low energies where one uses higher cumulants of the net proton distribution in order to find signals for a possible QCD critical point. Not only does the number of baryon and charges fluctuate due to the collision dynamics, so does the size of the system. And while ratios of cumulants do not depend on the average system size, they are affected by event by event fluctuation of the system size. This as been studied in [259] and it was found that only for the very most central collisions these fluctuations are suppressed. Therefore, any measurement of the centrality dependence of cumulant ratios needs to be interpreted with care. Alternatively, one can devise observables, which are not sensitive to size fluctuation [237, 241, 260, 261].

The first three points deserve some additional discussion, as they pose contradictory demands on the measurement [237]). In order to minimize corrections from global charge conservation, one wants to keep the acceptance window $\Delta$, say in rapidity, as small as possible. On the other hand, in order to capture the physics, the acceptance window needs to be sufficiently wide catch the correlation among the particles. Therefore, if $\sigma$ is the correlation length in rapidity and $\Delta_{\text {charge }}$ the range over which all the charges are distributed, then $\Delta / \Delta_{\text {charge }} \ll 1$ in order to minimize the effects of charge conservation, and $\sigma / \Delta \ll 1$ in order to capture the physics.

To illustrate this point, let consider the following schematic model. Let us define a two-particle correlation function (in rapidity $y$ )

$$
\left.\left\langle n\left(y_{1}\right)\left(n\left(y_{2}\right)-\delta\left(y_{1}-y_{2}\right)\right)\right)\right\rangle=\left\langle n\left(y_{1}\right)\right\rangle\left\langle n\left(y_{2}\right)\right\rangle\left(1+C\left(y_{1}, y_{2}\right)\right)
$$

Then the (acceptance dependent) scaled variance of the particle number is given by

$$
\frac{\left\langle\delta N^{2}\right\rangle}{\langle N\rangle}=1+\int_{-\Delta / 2}^{\Delta / 2} d y_{1} d y_{2} C\left(y_{1}, y_{2}\right)
$$

where the acceptance in rapidity is given by $-\Delta / 2<y<\Delta / 2$. Using a simple Gaussian for the correlation function

$$
C\left(y_{1}, y_{2}\right)=C_{0} \exp \left(-\frac{\left(y_{1}-y_{2}\right)^{2}}{2 \sigma}\right)
$$

in Fig. 19 we show the scaled variance as a function of the size of the acceptance window in units of the correlation length $\Delta / \sigma$. The black line is simply the 


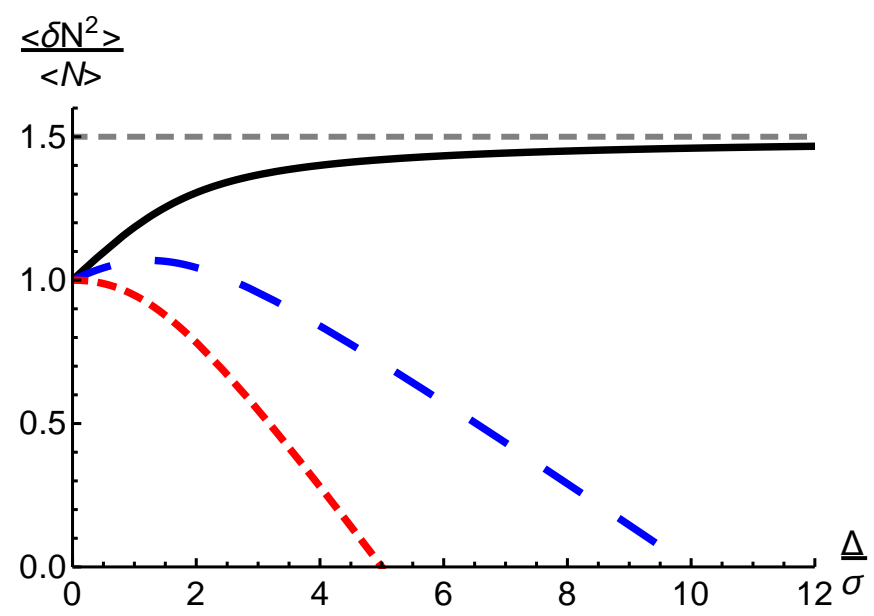

Figure 19: Observed scaled variance as a function of the acceptance window in units of the correlation length. The full (black) line corresponds to an infinite system where global charge conservation can be ignored. The long-dashed (blue) and short-dashed (red) line correspond the situation where the where the charge is conserved within $(-10 \sigma, 10 \sigma)$ and $(-5 \sigma, 5 \sigma)$, respectively.

expression of Eq. 62, where we have ignored any effects due to global charge conservation, i.e. $\Delta_{\text {charge }} \rightarrow \infty$. The red and blue dashed lines represent the situation where were the total charge is distributed over a range of $\Delta_{\text {charge }} / \sigma \leq 5$ and $\Delta_{\text {charge }} / \sigma \leq 10$, respectively. Here we used the leading order formulas of [262] to account for charge conservation noting that a more sophisticated treatment a la [263] would not change the picture qualitatively. Lattice QCD and model calculations, on the other hand would give the asymptotic value indicated by the dashed gray line, which we have chosen to be $\frac{\left\langle\delta N^{2}\right\rangle}{\langle N\rangle}=1.5$. The obvious lesson from this exercise is that a comparison of a measurement at one single acceptance window $\Delta$ with any model calculation is rather meaningless. Instead, one needs to measure the cumulants for various values of $\Delta$, and remove the effect of charge conservation. If the subsequent results trend towards an asymptotic value for large $\Delta$, it is this value which should be compared with model and lattice calculations. Such a program has been carried by the ALICE collaboration in order to extract the aforementioned charge fluctuations [264].

\subsection{Freeze-out conditions}

As we have discussed in Section 3 the hadron resonance gas is very successful in describing the (chemical) freeze out conditions of a heavy ion collision. In addition, with a few exceptions such as $C_{B S}$, lattice QCD calculations for the various cumulants agree very well with the HRG prediction for temperatures up to $T \sim 150 \mathrm{MeV}$. However, since the abundance of hadrons, such as the pion 
number, is not a well defined concept in an interacting thermal field theory, it would be desirable to extract the freeze out conditions by direct comparison of lattice QCD calculations with experimental data, thus, avoiding the, albeit successful, HRG model as an intermediate step. As first suggested in [265, 266] this goal can be achieved by comparing ratios of cumulants of conserved charges. The cumulants of the distribution of conserved charges are well defined in thermal field theory and they can, in principle, be measured in experiment, although the issues raised in the previous Section need to be resolved for such a comparison to be meaningful.

Since the cumulants depend both on the temperature and baryon number chemical potential, the two main parameters characterizing the chemical freeze out, a comparison with experiment should be able to constrain both of them. Following the specific strategy proposed in $[265,266]$ one first extracts the dependence of the cumulants on the chemical potential $\hat{\mu}_{X}=\left(\hat{\mu}_{B}, \hat{\mu}_{Q}, \hat{\mu}_{S}\right)$. Since the cumulants are derivatives of the pressure, Eq. (49), all that is needed is the pressure at finite chemical potential, which is given by the Taylor expansion, Eq. (50). For example, to leading order in the chemical potentials, the first order cumulant or the mean value of the net charge, $M_{Q}$, is given by

$$
\begin{aligned}
M_{Q}\left(T, \hat{\mu}_{B}, \hat{\mu}_{Q}, \hat{\mu}_{S}\right) & =\frac{1}{T^{4}} \frac{\partial}{\partial \hat{\mu}_{Q}} P\left(T, \hat{\mu}_{B}, \hat{\mu}_{Q}, \hat{\mu}_{S}\right) \\
& =\frac{1}{T^{4}} \frac{\partial}{\partial \hat{\mu}_{Q}}\left(P(T, 0)+\frac{\partial P}{\partial \hat{\mu}_{B}} \hat{\mu}_{B}+\frac{\partial P}{\partial \hat{\mu}_{Q}} \hat{\mu}_{Q}+\frac{\partial P}{\partial \hat{\mu}_{S}} \hat{\mu}_{S}\right) \\
& =\chi_{11}^{B Q} \hat{\mu}_{B}+\chi_{2}^{Q} \hat{\mu}_{Q}+\chi_{11}^{Q S} \hat{\mu}_{S} .
\end{aligned}
$$

and similar for the mean net baryon number $M_{B}$ and net strangeness $M_{S}$. Here, the cumulants in the last line are evaluated at vanishing chemical potential and thus are accessible to lattice QCD methods. Similar expressions can be derived for other, higher order cumulants.

In a heavy ion collision, no net strangeness is produced, i.e. $M_{S}=0$. Also, the ratio of electric charge over baryon number, $r=M_{Q} / M_{B}$, can be determined by experiment and is likely close to that of the incoming nuclei, $r \simeq 0.4$. These two constraints relate the charge and strangeness chemical potential to the baryon number chemical potential. Consequently, the cumulants depend only on the temperature and baryon number chemical potential, which then can be extracted from the comparison with experiment. In [266], the authors proposed to use the ratios of mean over variance and that of third order cumulant over variance for such a comparison

$$
R_{12}^{X}=\frac{M_{X}\left(T, \hat{\mu}_{B}\right)}{\sigma_{X}\left(T, \hat{\mu}_{B}\right)}, \quad R_{32}^{X}=\frac{\chi_{3}^{X}\left(T, \hat{\mu}_{B}\right)}{\sigma_{X}\left(T, \hat{\mu}_{B}\right)} .
$$



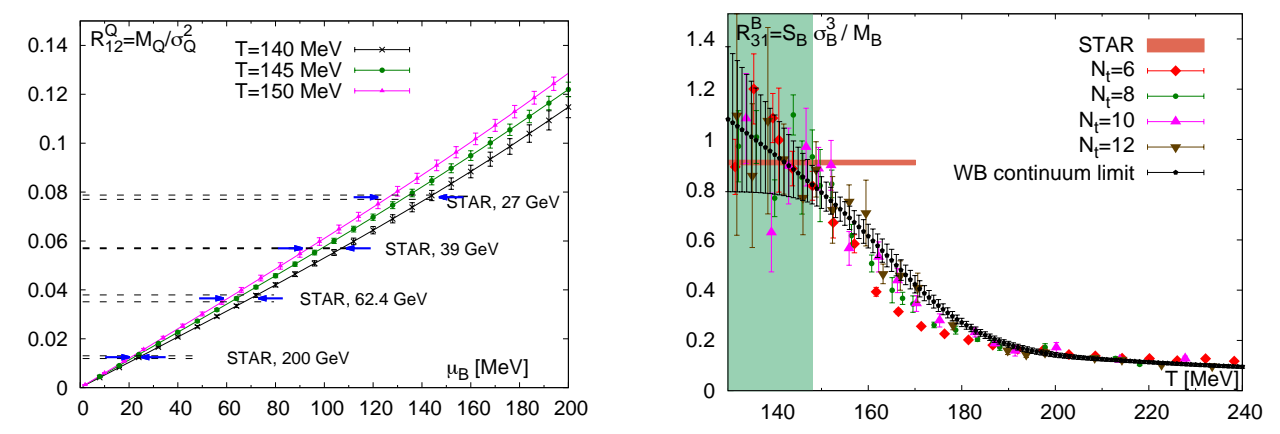

Figure 20: Comparison of lattice results with STAR data $[269,270]$ for $R_{12}^{Q}$ (left panel) and $R_{32}^{B}$ (right panel). Figures adapted from [268].

Here $X$ stands for charge or net baryon number. The first ratio, $R_{12}$ depends strongly on the baryon number chemical potential $\hat{\mu}_{B}$ whereas the $R_{32}$ has only a mild dependence on the $\hat{\mu}_{B}$, as can seen in the Boltzmann limit where $R_{12} \sim$ $\sinh \left(\hat{\mu}_{B}\right) / \cosh \left(\hat{\mu}_{B}\right) \sim \hat{\mu}_{B}$ and $R_{32} \sim \cosh \left(\hat{\mu}_{B}\right) / \cosh \left(\hat{\mu}_{B}\right)=1$. Therefore, $R_{12}$ constrains the chemical potential and $R_{32}$ the temperature. This is shown in Figs. 20 and 21 where we show the result of [267, 268], who used the methods of [266] together with their own lattice calculations in order to compare with the data of the STAR collaboration [269, 270]. While the chemical potential can be determined rather well from $R_{12}^{Q}$ (left panel of Fig. 20) and it agrees well with the HRG model (full line in Fig. 21), the large errors in the lattice calculations for $R_{32}^{B}$ allow only for an upper limit of the freeze-out temperature, $T_{f}<148 \mathrm{MeV}$ (right panel of Fig. 20 ). The freeze out temperature may be determined better if one additionally assumes that electric charge and baryon number freeze out at the same temperature. In this case the double ratio $R_{12}^{Q} / R_{12}^{B}$ constrains the freeze out temperature to within $T_{f}=144 \pm 6 \mathrm{MeV}$ [268]. Overall the extracted freeze out parameters from the comparison of cumulant ratios agree remarkably well with those obtained from the HRG analysis.

With regards to our discussion in the previous Section, we note that the measured cumulants have not been corrected for global charge and baryon number conservation and have been obtained for a fixed acceptance window. In addition, the experiment measures the net proton cumulants which are compared with net baryon cumulants from the lattice. Therefore, the fact that the freeze out parameters agree rather well with those obtained from and HRG analysis, is somewhat surprising. However, if the system at freeze-out approximately follows Poisson statistics, i.e. correlations are negligible, as is the case in the HRG model, then the lack of neutron detection and the effect of the acceptance window cancel 


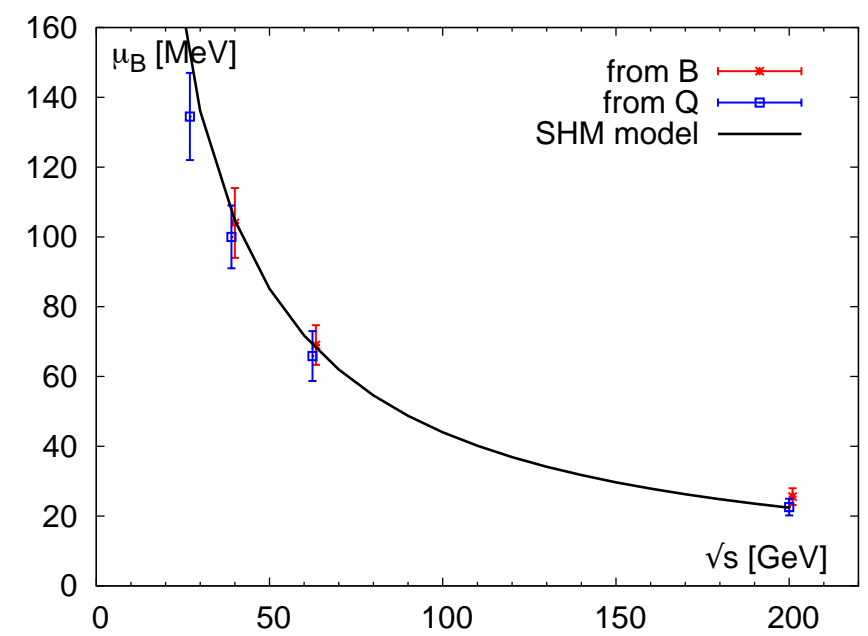

Figure 21: Comparison of lattice results with STAR data $[269,270]$ for charge and baryon number fluctuations. In this figure we show the extracted freeze out chemical potential in comparison with the HRG model (full line). Figures adapted from [268].

in the ratios and we recover the HRG values. A recent comparison of data taken at the LHC by the ALICE collaboration with lattice cumulants seems to support this possibility [271]. In this paper, the authors assumed that the net-charges follow a Skellam distribution, i.e. absence of any correlations. The various cumulants are then determined by combinations of mean values, and, using the particle yields measured by ALICE, they found a very good agreement with the lattice calculation. However, only a careful analysis of the experimental data along the lines discussed above will be able to verify, if indeed the particles at freeze out follow Poisson statistics.

\subsection{Fluctuations and the QCD Phase diagram}

The phase diagram of QCD has potentially a very rich structure, see Fig. 1. As discussed in Section 2.2 one of the more remarkable aspects of the phase diagram is the possible existence of a critical endpoint of the chiral transition. The idea about a possible co-existence region and critical point has spawned a dedicated program at the Relativistic Heavy Ion Collider (RHIC) at Brookhaven National Laboratory, where the so called beam energy scan (BES) tries to scan the phase diagram by colliding nuclei over the whole range of collision energies available at RHIC. As discussed in Section 3, by lowering the beam energy one increases the baryon number chemical potential of the system created in these collisions, and the energy range of RHIC allows to scan the phase diagram over 
the range of $0 \lesssim \mu_{B} \lesssim 400 \mathrm{MeV}[45]$.

The measurement of fluctuations, in particular cumulants of the net baryon number, play a central role in the experimental search for a possible critical point. At the critical point, where we have a second order phase transition, the correlation length $\xi$ diverges for a static system of infinite size, resulting in diverging cumulants of the baryon number $[68,272]$. However, the system created in a heavy ion collision has finite size as well as a finite lifetime which limits the maximum correlation length, and, as shown in [273], due to critical slowing down, it is the finite lifetime which restricts the correlation length to $\xi \simeq 2$ $\mathrm{fm}$. Thus, instead on diverging susceptibilities, one will only observe moderate enhancements. These enhancements are larger for higher order cumulants, as pointed out in [274] where it was shown that the fourth order cumulant scales with the seventh power of the correlation length $\chi_{4}^{B} \sim \xi^{7}$, while the second order scales like the square $\chi_{B} \sim \xi^{2}$. Consequently the ratio, $R_{42}=\chi_{4}^{B} / \chi_{2}^{B} \sim \xi^{5}$ grows with a large power of $\xi$.

More recently is was realized that in the region around the critical point $R_{42}$ may be enhanced or reduced, and that a typical trajectory of freeze-out points as a function of beam energy would suggest the following scenario: With decreasing beam energy $R_{42}$ is initially below the Poisson baseline $R_{42}^{\text {Poisson }}=1$, then rises above it, and finally returns to the baseline value $R_{42}=1$ as kinetic freeze-out occurs outside the critical region in the hadronic phase [275, 276]. A first measurement by the STAR collaboration of the beam energy dependence of various cumulant ratios [269] indeed showed a small decrease below the baseline for energies $\sqrt{s}<20 \mathrm{GeV}$. However, this observation may not be significant because a similar reduction is also seen in hadronic event-generators, and thus may simply be an effect of baryon number conservation. On the other hand, recent preliminary data, which also include protons at higher transverse momentum, show a small dip followed by a large increase at the lowest beam energy $\sqrt{s}=7.7 \mathrm{GeV}[255]$. Although the data have significant statistical errors, these results are rather intriguing and beg for a measurement at even lower energies. While statistics will be improved during the second phase of the RHIC beam energy scan, a measurement at energies below $\sqrt{s}=7.7 \mathrm{GeV}$ will have to be carried out at another facility. One should also point out that the enhancement of $R_{42}$ observed in the preliminary STAR data is due to protons with transverse momentum $p_{t}>800 \mathrm{MeV}$ and that the enhancement increases with increased rapidity coverage. While the latter is to be expected following our discussion in Section 5.2, the former requires more scrutiny, since naively one would expect critical fluctuations to arise from low momentum modes.

Finally, we should remark that a first order transition will also give rise to enhanced fluctuation of the baryon number. Especially if one enters the mechanically unstable spinodal region, as it is possible in a dynamic process such as 
a heavy ion collision. The associated instabilities give rise to the rapid formation of blobs of high density matter [277], which should reflect itself in enhanced fluctuations of the baryon number. While the formation of such blobs of matter has been demonstrated in model calculations [278, 279], their effect on baryon number cumulants has not yet been investigated quantitatively.

Aside from exploring the phase diagram at large baryon density, fluctuation measurements also allow to experimentally address the nature of the cross-over transition at vanishing baryon number chemical potential. It is commonly believed that the cross-over transition is a remnant of the $O(4)$ chiral transition which in the limit of vanishing quark or pion masses would be of second order [59]. In this case, one can make quite general predictions about the baryon cumulants at vanishing chemical potential, as discussed in some detail e.g. in [280]. Essential for this to work is the fact that the so-called pseudo critical line (black dashed line in Fig. 1) defined by the inflection point of the chiral condensate $\langle\bar{\psi} \psi\rangle[37,38]$, has a finite curvature, $T_{p c}\left(\mu_{B}\right)=T_{p c}\left(\mu_{B}=0\right)\left[1-\kappa \hat{\mu}_{B}^{2}\right]$ with $\kappa=0.0066[281,282]$. Consequently, close to the pseudo-critical line one may write the free energy as a function of an effective reduced "temperature" $t=\left[\left(T-T_{c}\right) / T_{c}+\kappa \hat{\mu}_{B}^{2}\right]$

$$
F\left(T, \mu_{B}\right)=F(t)
$$

As a result, derivatives with respect to the chemical potential can be can be related with derivatives with respect to the temperature. Since the effective temperature has quadratic dependence on $\hat{\mu}_{B}$, the $n$-th derivative with respect to $\hat{\mu}_{B}$ corresponds to a derivative of order $n / 2$ with respect to the temperature. For example,

$$
\frac{\partial^{2}}{\partial \hat{\mu}_{B}^{2}} F=2 T \kappa \frac{\partial}{\partial T} F
$$

With this relation one can then apply the results of $O(4)$ scaling theory directly to the baryon number cumulants, which in turn can be tested by experiment. One of the predictions is, for example, that the ratio of the sixth over the second order cumulant is negative right below the freeze out temperature, $R_{62}^{B}\left(T=T_{f}\right)<$ 0 , which in principle is accessible to experiments at the LHC. For a detailed discussion we refer to [280].

\section{Hadrons in a hot and dense medium}

\subsection{Chiral effective theory}

Penetrating probes like photons and dileptons can be used to test the quasiparticle structure of hot and dense matter. In the following we will concentrate on 
the regime below the phase transition, where the relevant degrees of freedom are hadrons. At low temperature and small baryon density the dominant degrees of freedom are pions. Pions are Goldstone bosons associated with the spontaneously broken chiral symmetry, and their interactions are constrained by the underlying symmetry.

The Goldstone boson fields can be parameterized by unitary matrices $\Sigma=$ $\exp \left(i \lambda^{a} \phi^{a} / f_{\pi}\right)$ where $\lambda^{a}$ are the Gell-Mann matrices for $S U(3)$ flavor and $f_{\pi}=93$ $\mathrm{MeV}$ is the pion decay constant. For example, $\pi^{0}=\phi^{3}$ and $\pi^{ \pm}=\left(\phi_{1} \pm i \phi_{2}\right) / 2$ describe the neutral and charged pion. Other components of $\phi^{a}$ describe the neutral and charged kaons, as well as the eta. The eta prime acquires a large mass because of the axial anomaly, and is not a Goldstone boson.

At low energy the effective Lagrangian for $\Sigma$ can be organized as an expansion in the number of derivatives of $\Sigma$. This is the case because higher derivative terms describe interactions that scale as either the momentum or the energy of the Goldstone boson. Since Goldstone bosons are approximately massless, the energy is of the same order of magnitude as the momentum. We will see that the expansion parameter is $p /\left(4 \pi f_{\pi}\right)$. At leading order in $\left(\partial / f_{\pi}\right)$ there is only one structure which is consistent with chiral symmetry, Lorentz invariance and $\mathrm{C}, \mathrm{P}, \mathrm{T}$. This is the Lagrangian of the non-linear sigma model. In the presence of a small explicit symmetry breaking term we have

$$
\mathcal{L}=\frac{f_{\pi}^{2}}{4} \operatorname{Tr}\left[\partial_{\mu} \Sigma \partial^{\mu} \Sigma^{\dagger}\right]+\left[B \operatorname{Tr}\left(M \Sigma^{\dagger}\right)+\text { h.c. }\right]+\ldots
$$

Here, $M=\operatorname{diag}\left(m_{u}, m_{d}, m_{s}\right)$ is the quark mass matrix and $B$, the coefficient of the symmetry breaking term, is a low energy constant that we will fix below. In order to show that the parameter $f_{\pi}$ is related to the pion decay amplitude we have to gauge the non-linear sigma model. This is achieved by introducing the gauge covariant derivative $\nabla_{\mu} \Sigma=\partial_{\mu} \Sigma+i g_{w} W_{\mu} \Sigma$ where $W_{\mu}$ is the charged weak gauge boson and $g_{w}$ is the weak coupling constant. The gauged non-linear sigma model gives a pion- $W$ boson interaction $\mathcal{L}=g_{w} f_{\pi} W_{\mu}^{ \pm} \partial^{\mu} \pi^{\mp}$. This term leads to an amplitude for the decay $\pi^{ \pm} \rightarrow W^{ \pm} \rightarrow e^{ \pm} \nu_{e}$ or $\pi^{ \pm} \rightarrow W^{ \pm} \rightarrow \mu^{ \pm} \nu_{\mu}$ which is proportional to $g_{w} f_{\pi} q_{\mu}$, where $q_{\mu}$ is the momentum of the pion. This result agrees with the standard definition of $f_{\pi}$ in terms of the pion-weak axial current matrix element. In the ground state $\Sigma=1$ and the ground state energy is $E_{\text {vac }}=-2 B \operatorname{Tr}[M]$. Using the relation $\langle\bar{q} q\rangle=\partial E_{\text {vac }} /(\partial m)$ we find $\langle\bar{q} q\rangle=-2 B$. Fluctuations around $\Sigma=1$ determine the Goldstone boson masses. The pion mass satisfies the Gell-Mann-Oaks-Renner relation (GMOR) [54]

$$
m_{\pi}^{2} f_{\pi}^{2}=\left(m_{u}+m_{d}\right)\langle\bar{q} q\rangle
$$

and analogous relations exist for the kaon and eta masses. 
Corrections to this result arise from higher derivative corrections to the effective lagrangian Eq. (68), and from loop corrections computed using the leading order vertices. Expanding out Eq. (68) to fourth order in $\phi^{a}$ gives interaction terms of the form $\mathcal{L} \sim f_{\pi}^{-2}\left(\phi^{a} \partial_{\mu} \phi^{a}\right)^{2}$. This means that the tree level mesonmeson interaction scales as $q^{2} / f_{\pi}^{2}$, and that the low energy interaction is indeed weak. Computing loop diagrams gives corrections to this result that contain additional factors of $q /\left(4 \pi f_{\pi}\right)$. The numerical factor $1 /(4 \pi)$ arises from the phase space in loop integrals, and clearly helps in obtaining meaningful results for pion momenta up to a few hundred $\mathrm{MeV}$.

Higher order gradient corrections involve terms like $\mathcal{L} \sim c_{4} \operatorname{Tr}\left[\left(\partial_{\mu} \Sigma \partial^{\mu} \Sigma^{\dagger}\right)^{2}\right]$. On dimensional grounds $c_{4}$ is suppressed by a factor $f_{\pi}^{-2}$ relative to the leading order interaction. Since $c_{4}$ acts as a counter-term that can be used to absorb the scale dependent pieces of one-loop terms a more accurate estimate is $c_{4} f_{\pi}^{-2} \sim$ $\left(4 \pi f_{\pi}\right)^{-2}$. Alternatively, we can view higher order gradient terms as arising from integrating out resonances like the rho meson and the $K^{*}$. This suggests $c_{4} f_{\pi}^{-2} \sim m_{V}^{-2}$, where $V=\rho, K^{*}, \ldots$. We note that $m_{V}$ is numerically close to the scale $4 \pi f_{\pi} \simeq 1160 \mathrm{MeV}$, implying that the values of $f_{\pi}$ and the vector meson masses in QCD are natural.

\subsection{Chiral effective theory at finite temperature}

As a first application of the chiral Lagrangian we study the dependence of the chiral condensate on the temperature. Like the GMOR relation this result can be extracted from thermodynamic properties. At finite $T$ we have to consider the free energy $F=E-T S$ instead of the energy $E$. At leading order in $T / f_{\pi}$, we can ignore interactions between pions. The free energy of an ideal pion gas is

$$
F=\left(N_{f}^{2}-1\right) T \int \frac{d^{3} p}{(2 \pi)^{3}} \log \left(1-e^{-E_{\pi} / T}\right),
$$

where $E_{\pi}=\sqrt{p^{2}+m_{\pi}^{2}}$. The quark condensate is $\langle\bar{q} q\rangle=\left(N_{f}\right)^{-1} \partial F / \partial m$. Equation (70) depends on the quark mass only through the pion mass. Using the Gell-Mann-Oakes-Renner relation (69) we find [283]

$$
\langle\bar{q} q\rangle_{T}=\langle\bar{q} q\rangle_{0}\left\{1-\frac{N_{f}^{2}-1}{3 N_{f}}\left(\frac{T^{2}}{4 f_{\pi}^{2}}\right)+\ldots\right\} .
$$

This result shows that there is a tendency towards chiral symmetry restoration already at low temperature, and that the relevant scale is set by $T \sim 2 f_{\pi} \sim 180$ $\mathrm{MeV}$. There is a nice physical interpretation of the result given in Eq. (71). The chiral condensate in vacuum is negative, but the pion matrix elements of $m_{q} \bar{q} q$ is positive. A finite density of thermal pions therefore reduces the vacuum condensate, or, to quote Gerry Brown, "they act as a vacuum cleaner" 
Another simple application of chiral perturbation theory involves the vector and axial-vector correlation functions. The correlator of the vector current can be accessed using di-lepton measurements, see Section 7, and the difference between the vector and axial-vector correlators is a measure of chiral symmetry breaking. The correlation functions are defined by

$$
\Pi_{\mu \nu}^{V, A}(q)=-i \int d^{4} x \Theta\left(x_{0}\right) e^{i q \cdot x}\left\langle\left[j_{\mu}^{V, A}(x), j_{\nu}^{V, A}(0)\right]\right\rangle
$$

where $j_{\mu}^{V}=\bar{q} \frac{\tau^{a}}{2} \gamma_{\mu} q$ and $j_{\mu}^{A}=\bar{q} \frac{\tau^{a}}{2} \gamma_{\mu} \gamma_{5} q$ are the vector and axial-vector currents, and we have suppressed the isospin indices $a, b$ on the correlation function. The functions in Eq. (72) are retarded correlation functions. As usual, the spectral function is determined by the imaginary part of the retarded correlator. A set of sum rules for the vector and axial-vector spectral functions was derived by Weinberg. We can split the correlators into transverse and longitudinal parts

$$
\Pi_{\mu \nu}(q)=\Pi_{T}\left(q^{2}\right) P_{\mu \nu}^{T}+\Pi_{L}\left(q^{2}\right) P_{\mu \nu}^{L},
$$

with $P_{\mu \nu}^{T}=q_{\mu} q_{\nu} / q^{2}-g_{\mu \nu}$ and $P_{\mu \nu}^{L}=q_{\mu} q_{\nu} / q^{2}$. We define $\rho^{V, A}(s)=\frac{1}{\pi} \operatorname{Im} \Pi_{T}^{V, A}(s)$. Then the Weinberg sum rules in the chiral limit read [284]

$$
\begin{aligned}
& \int_{0}^{\infty} \frac{d s}{s}\left(\rho_{V}(s)-\rho_{A}(s)\right)=f_{\pi}^{2}, \\
& \int_{0}^{\infty} d s\left(\rho_{V}(s)-\rho_{A}(s)\right)=0 .
\end{aligned}
$$

Additional sum rules, and corrections due to finite quark masses can be studied using the operator product expansion [285]. The sum rules provide an explicit relation between the difference of the spectra in the vector and axial-vector channels and spontaneous chiral symmetry breaking, controlled by $f_{\pi}$. The Weinberg sum rules can be extended to finite temperature provided we interpret the sum rules as integrals over energy at fixed momentum, and separate out the pion contribution more carefully [286]. At lowest order in the chiral expansion one can show that the finite temperature correlators are related to the $T=0$ functions by the simple mixing relation [287]

$$
\Pi_{\mu \nu}^{V, A}(q)=(1-\epsilon) \Pi_{\mu \nu, 0}^{V, A}+\epsilon \Pi_{\mu \nu, 0}^{A, V}(q),
$$

with $\epsilon=T^{2} /\left(6 f_{\pi}^{2}\right)$ and the subscript 0 refers to the $T=0$ result. This formula, too, has a simple interpretation. In a thermal medium the vector current can couple to thermal pions and mix with the axial current, and vice versa.

In order to make more quantitative statements about the vector current spectral function we have to understand the coupling of the vector current to hadronic 
states, in particular the $\rho$ meson. The $\rho$ meson is not a Goldstone boson, and these calculations inevitably involve model assumptions. A successful scheme for constructing effective Lagrangians for vector mesons is the massive YangMills scheme [288]. Here, we assume that the $\rho$ and $a_{1}$ mesons are vector and axial-vector gauge fields associated with the $S U(2)_{L} \times S U(2)_{R}$ symmetry of the non-linear sigma model. The gauge symmetry is broken by a Higgs field that gives masses to the $\rho$ and $a_{1}$. A different scheme, known as the hidden local symmetry scheme, was introduced by Bando, Kugo, and Yamawaki [289], and elaborated by many others, see [290].

In the massive Yang-Mills scheme the leading interaction between pions, rho mesons, and the $a_{1}$ is given by

$$
\begin{aligned}
\mathcal{L}= & \frac{1}{2} m_{\rho}^{2} \vec{\rho}_{\mu}^{2}+\frac{1}{2} m_{a_{1}}^{2} \vec{a}_{1 \mu}^{2}+g^{2} f_{\pi} \vec{\pi} \times \vec{\rho}^{\mu} \cdot \vec{a}_{1, \mu} \\
& +g_{\rho \pi \pi}\left(\vec{\rho}_{\mu}^{2} \vec{\pi}^{2}-\vec{\rho}^{\mu} \cdot \vec{\pi} \vec{\rho}_{\mu} \cdot \vec{\pi}\right)+g_{\rho \pi \pi} \vec{\rho}^{\mu} \cdot\left(\vec{\pi} \times \partial^{\mu} \vec{\pi}\right)+\ldots
\end{aligned}
$$

where $2 g_{\rho \pi \pi}^{2}=g^{2}$ and the Higgs mechanism leads to the mass formula $m_{a_{1}}^{2}=$ $m_{\rho}^{2}+g^{2} f_{\pi}^{2}$. The massive Yang-Mills Lagrangian exhibits vector meson dominance, that means the coupling of the isospin-one component of the photon to the vector current is saturated by the rho meson. As a result, the calculation of the vector spectral function can be reduced to computing the self-energy of the rho meson [291]. The main contributions at $T=0$ are the $\pi \pi$ and $\pi a_{1}$ intermediate states. Both of these receive corrections at finite temperature due to thermal pion states. At low temperature these thermal effects reproduce the mixing formula in Eq. (76).

In a dense hadron gas higher resonances become important. There are, in particular, many nucleon resonances $N^{*}$ that have a strong coupling to $N \rho$ [292]. In the $\rho$ self energy this corresponds to intermediate states of the form $N^{*} N^{-1}$. In vacuum excited-nucleon anti-nucleon intermediate states are strongly suppressed, but in a medium with non-zero baryon density we find excited-nucleon nucleonhole states that make significant contributions. Also, at finite temperature there is a non-zero thermal population of baryons and anti-baryons. The net effect of these contributions is a significant broadening of the $\rho$, together with extra strength at low invariant mass [291].

Similar effects have been studied in nuclear physics for a long time. In finite nuclei and nuclear matter pions (or states with the quantum numbers of pions) can mix with nucleon-hole and delta-hole pairs [293]. The p-wave $\pi N \Delta$ interaction is quite strong, and these effects can lead to a softening of the pion dispersion relation, which is a precursor of a possible pion condensed phase in cold dense nuclear matter. The Delta-hole mechanism is not restricted to cold nuclear phases, and possible effects in heavy ion collisions in the fixed target regime where studied in $[48,294]$. 
Brown and Rho suggested that, because of the chiral and scale symmetries of the QCD Lagrangian, the complicated many-body dynamics of quarks and gluons can be summarized in terms of simple scaling laws for the effective masses of hadrons [49]. They proposed that

$$
\frac{m_{\rho}^{*}}{m_{\rho}}=\frac{m_{a_{1}}^{*}}{m_{a_{1}}}=\left(\frac{\langle\bar{q} q\rangle_{\rho, T}}{\langle\bar{q} q\rangle_{0}}\right)^{1 / 3} .
$$

where $m_{\rho}^{*}$ and $m_{a_{1}}^{*}$ are the in-medium masses. In this scenario chiral symmetry is restored because all hadrons become massless at the critical temperature. This is different from the picture discussed above, where chiral symmetry is restored because of the effects of mixing between chiral partner channels, combined with the melting of hadronic resonances. The Brown-Rho scenario was investigated in great detail by analyzing the spectra of dileptons emitted in heavy ion collisions at different energies, see Section 7 .

\subsection{Quasiparticles in the quark-gluon plasma}

We can also identify quasi-particles in the high temperature phase. In Section 2.2 we noted that in the quark-gluon plasma the color Coulomb interaction is screened at a distance $r \sim m_{D}^{-1}$, where

$$
m_{D}^{2}=g^{2}\left[\left(1+\frac{N_{f}}{6}\right) T^{2}+\frac{N_{f}}{2 \pi^{2}} \mu^{2}\right]
$$

is called the Debye mass. In perturbation theory the static magnetic interaction is unscreened [164], but non-static magnetic interactions are dynamically screened at a distance $r \sim\left(m_{D}^{2} \omega\right)^{-1 / 3}$. This phenomenon, known as Landau damping, is due to the coupling of gluons to particle-hole (or particle-anti-particle) pairs, and also play a role in electromagnetic plasmas. Unlike classical plasmas the QCD plasma has a non-perturbative static magnetic screening mass $m_{M} \sim g^{2} T$. This is the scale that determines the non-perturbative contributions to the pressure. Modes below the magnetic screening scale contribute

$$
P \sim T \int^{m_{M}} d^{3} q \sim g^{6} T^{4}
$$

which implies that the "last" perturbatively calculable contribution to the QGP pressure is $O\left(g^{6} \log (g)\right)$ [83].

The electric screening scale also determines the properties of collective gluonic modes, see [295]. For momenta $q \gg g T$ there are two transverse modes with dispersion relation $\omega \simeq q$. For momenta $q<g T$ there are two transverse and one longitudinal mode. The longitudinal mode is sometimes called the plasmon. The energy of all three modes approaches $\omega=\omega_{p}=m_{D} / \sqrt{3}$ as $q \rightarrow 0$. The quantity 
$\omega_{p}$ is known as the plasma frequency. The gluon (and plasmon) decay constant in the limit $q \rightarrow 0$ is [296]

$$
\gamma=6.64 \frac{g^{2} N_{c} T}{24 \pi}
$$

which confirms that the quasi-particle width is parametrically small compared to their energy. Numerically, the ratio of the plasmon width to the plasmon energy is $\gamma / \omega_{p} \simeq g / 2.2$, which is of order one even in a weakly coupled plasma. However, as discussed in Section 2.3, models based on screened quasi-particles are successful in describing the thermodynamics of QCD down to $T \sim 2 T_{c}$. The calculation of the collisional width of quasi-particles with momenta of order $T$ is a complicated, non-perturbative problem, but the width remains parametrically small, $\gamma \sim g^{2} \log (1 / g) T[297]$.

Quarks also form collective modes by coupling to gluons. Perturbative interactions do not contribute to chiral symmetry breaking mass terms that connect left and right-handed. However, perturbative interaction can generate an effective energy, and lead to mixing between chirality and helicty eigenstates. It is standard to define an effective fermion "mass"

$$
m_{f}^{2}=\frac{g^{2}}{6}\left[T^{2}+\frac{\mu^{2}}{\pi^{2}}\right] .
$$

There are two fermionic branches, one with helicity equal to chirality, and another, called the plasmino, in which the two quantities are opposite. Both modes satisfy $\omega \sim m_{f}$ for small momenta. At large momenta the plasmino disappears, and the standard quark mode satisfies $\omega \simeq q+m_{f}^{2} / q$.

At very low momenta the only propagating mode is the hydrodynamic sound modes discussed in Section 4. Sound is damped by shear and bulk viscosity. The width of a sound mode with energy $\omega=c_{s} q$ is given by $\gamma=\left[c_{s} \Gamma_{s} q^{3}\right]^{1 / 2}$ with

$$
\Gamma_{s}=\frac{1}{s T}\left(\frac{4 \eta}{3}+\zeta\right)
$$

Using the perturbative result for $\eta / s$ given in Eq. (32) we conclude that $\Gamma_{s} \sim$ $1 /\left(g^{4} T\right)$, and only very low momentum sound modes with $q \lesssim g^{4} T$ can propagate. In a nearly perfect fluid, on the other hand, modes with $q \sim T$ are propagating. Numerically, the width over energy of a sound mode is $\gamma / \omega \simeq[2(\eta / s)(\omega / T)]^{1 / 2}$. For $\eta / s \simeq 1$ only modes with $\omega \lesssim 0.5 T$ are propagating, but in a nearly perfect fluid we find a much less restrictive bound, $\omega \lesssim 6 T$.

\subsection{Quasiparticles in dense quark matter}

Finally, quasi-particle properties can be studied in the regime of very high baryon density. Above the critical temperature for color superconductivity we 
have quasi-quarks and quasi-gluons with the effective masses given in Eq. (79) and (82). The perturbative expansion has some unusual features, known as nonFermi liquid behavior [298], but quasi-particles are well-defined and have small widths. At $T_{c}$ quarks acquire a gap, and gluons acquire magnetic screening masses by a QCD analog of the Meissner effect. The situation is simplest in the CFL phase, where all quarks and gluons acquire a gap. The gap in the quark sector was determined by Son [299],

$$
\Delta_{C F L} \simeq 2^{4 / 3} \Lambda_{B C S} \exp \left(-\frac{\pi^{2}+4}{8}\right) \exp \left(-\frac{3 \pi^{2}}{\sqrt{2} g}\right)
$$

with $\Lambda_{B C S}=256 \pi^{4}\left(2 / N_{f}\right)^{5 / 2} g^{-5} \mu$ [300]. The nine different color-flavor combinations organize themselves into an octet with gap $\Delta_{C F L}$, and a singlet with gap $2 \Delta_{C F L}[75]$.

The CFL order parameter breaks chiral symmetry, and for energies below the gap the propagating modes are Goldstone bosons. Based on symmetry arguments, the effective Lagrangian has the same structure as the Lagrangian of chiral perturbation theory, Eq. (68), except that Lorentz-invariance is no longer a symmetry. We have [301]

$$
\mathcal{L}_{\text {eff }}=\frac{f_{\pi}^{2}}{4} \operatorname{Tr}\left[\nabla_{0} \Sigma \nabla_{0} \Sigma^{\dagger}-v_{\pi}^{2} \partial_{i} \Sigma \partial_{i} \Sigma^{\dagger}\right]
$$

where the speed of Goldstone modes is $v_{\pi}^{2} \simeq 1 / 3$ [302]. If quark masses are taken into account then the Goldstone bosons acquire small masses, $m_{\pi}, m_{K} \ll \Delta$. It interesting to consider the properties of gapped quasi-quarks in more detail. We already noted that quarks are organized into an octet and a singlet of the $S U(3)$ flavor group. We also find that quasi-particles have integer electric and baryon charges. These arise from the diquark polarization cloud that surrounds a single quark, and the phenomenon can be described as quark-hadron complementary [78]. The effective Lagrangian for fermions in the CFL phase can be written as [303]

$$
\begin{aligned}
\mathcal{L}= & \operatorname{Tr}\left(N^{\dagger} i v^{\mu} D_{\mu} N\right)-D \operatorname{Tr}\left(N^{\dagger} v^{\mu} \gamma_{5}\left\{\mathcal{A}_{\mu}, N\right\}\right)-F \operatorname{Tr}\left(N^{\dagger} v^{\mu} \gamma_{5}\left[\mathcal{A}_{\mu}, N\right]\right) \\
+ & \frac{\Delta}{2}\left\{\left(\operatorname{Tr}\left(N_{L} N_{L}\right)-\left[\operatorname{Tr}\left(N_{L}\right)\right]^{2}\right)-(L \leftrightarrow R)+\text { h.c. }\right\} .
\end{aligned}
$$

$N_{L, R}$ are left and right handed baryon fields in the adjoint representation of flavor $S U(3)$. We can think of $N$ as describing a quark which is surrounded by a diquark cloud, $N_{L} \sim q_{L}\left\langle q_{L} q_{L}\right\rangle$. The covariant derivative of the nucleon field is given by $D_{\mu} N=\partial_{\mu} N+i\left[\mathcal{V}_{\mu}, N\right]$, and $\mathcal{V}_{\mu}$ and $\mathcal{A}_{\mu}$ are the pionic (and kaonic) vector and axial-vector currents. The coupling constants $D, F$ control the axial-vector couplings in flavor $S U(3)$, and the last line in Eq. (86) is a Majorana mass term 
that describes the flavor structure of the gap. What is interesting about Eq. (86) is that, except for the gap, this Lagrangian has the same structure as the flavor $S U(3)$ chiral Lagrangian for baryons at zero temperature and density, which illustrates the possibility of a continuous phase transition between (hyperonic) nuclear matter and strange quark matter [78].

\subsection{Landau Fermi liquid theory}

An important issue related to the modification of quasi-particle properties in a hot and dense medium is the consistent treatment of hadronic transport properties. This problem does not arise in the context of fluid dynamics, where the only relevant properties are the equation of state and a small set of transport coefficients, but it is an issue in hadronic transport models. The simplest example of a theory that provides a consistent treatment of single particle and transport properties is the Landau theory of Fermi liquids, which is directly applicable to cold and dense systems of baryons. Consider a cold Fermi system in which the low energy excitations are spin $1 / 2$ quasi-particles. Landau proposed to define a distribution function $f_{p}=f_{p}^{0}+\delta f_{p}$ for the quasi-particles. Here, $f_{p}^{0}$ is the ground state distribution function, and $\delta f_{p} \ll f_{p}^{0}$ is a correction. The energy density can be written as $[171,304,305,306]$

$$
\mathcal{E}=\mathcal{E}_{0}+\int d \Gamma_{p} \frac{\delta \mathcal{E}}{\delta f_{p}} \delta f_{p}+\frac{1}{2} \iint d \Gamma_{p} d \Gamma_{p^{\prime}} \frac{\delta^{2} \mathcal{E}}{\delta f_{p} \delta f_{p^{\prime}}} \delta f_{p} \delta f_{p^{\prime}}+\ldots
$$

with $d \Gamma_{p}=d^{3} p /(2 \pi)^{3}$. Functional derivatives of $\mathcal{E}$ with respect to $f_{p}$ define the quasi-particle energy $E_{p}$ and the effective interaction $t_{p p^{\prime}}$

$$
E_{p}=\frac{\delta \mathcal{E}}{\delta f_{p}} \quad t_{p p^{\prime}}=\frac{\delta^{2} \mathcal{E}}{\delta f_{p} \delta f_{p^{\prime}}} .
$$

Note that, in general, $E_{p}$ is a non-trivial function of the distribution function $f_{p}(x, t)$. This implies, in particular, that the particles have density and temperature dependent effective masses. Near the Fermi surface we can write $E_{p}=v_{F}\left(|\vec{p}|-p_{F}\right)$, where $v_{F}$ is the Fermi velocity, $p_{F}$ is the Fermi momentum, and $m^{*}=p_{F} / v_{F}$ is the effective mass. We can decompose $t_{p p^{\prime}}=F_{p p^{\prime}}+G_{p p^{\prime}} \vec{\sigma}_{1} \cdot \vec{\sigma}_{2}$. On the Fermi surface the effective interaction is only a function of the scattering angle and we can expand the angular dependence as

$$
F_{p p^{\prime}}=\sum_{l} F_{l} P_{l}\left(\cos \theta_{\vec{p} \cdot \vec{p}^{\prime}}\right)
$$

where $P_{l}(x)$ is a Legendre polynomial, and $G_{p p^{\prime}}$ can be expanded in an analogous fashion. The coefficients $F_{l}$ and $G_{l}$, which control the properties of quasi-particles, are called Landau parameters. There are a number of interesting connections 
between single-particle and collective properties. For example, the effective mass is

$$
m^{*}=m\left(1+\frac{F_{1}}{3}\right),
$$

and the speed of sound is given by

$$
c_{s}^{2}=\frac{v_{F}^{2}}{3} \frac{1+F_{0}}{1+F_{1} / 3},
$$

where $v_{F}$ is the Fermi velocity defined above.

The distribution function satisfies a Boltzmann equation

$$
\left(\partial_{t}+\vec{v}_{p} \cdot \vec{\nabla}_{x}+\vec{F}_{p} \cdot \vec{\nabla}_{p}\right) f_{p}(x, t)=C\left[f_{p}\right]
$$

where $\vec{v}_{p}=\vec{\nabla}_{p} E_{p}$ is the quasi-particle velocity, $\vec{F}_{p}=-\vec{\nabla}_{x} E_{p}$ is an effective force, and $C\left[f_{p}\right]$ is the collision term. Conserved currents can be defined in terms of $f_{p}$ and the single particle properties $E_{p}$ and $v_{p}$. For example, we can write the mass density $\rho$ and mass current $\vec{\jmath}$ as

$$
\rho=\int d \Gamma_{p} m f_{p}, \quad \vec{\jmath}=\int d \Gamma_{p} m \vec{v}_{p} f_{p}
$$

where $d \Gamma_{p}=d^{3} p /(2 \pi)^{3}$. The Boltzmann equation implies that the current is conserved, $\partial_{0} \rho+\vec{\nabla} \cdot \vec{\jmath}=0$. Since $E_{p}$ and $v_{p}$ are functionals of the particle distribution $f_{p}(x, t)$, the validity of conservation laws is non-trivial. In the framework of Landau Fermi liquid theory, conservation laws follow from the condition that $E_{p}$ can be derived from an energy density functional, see Eq. (88). The equation of momentum conservation is $\partial_{0} \pi_{i}+\nabla_{j} T_{i j}=0$, where

$$
T_{i j}(\vec{x}, t)=\int d \Gamma_{p} p_{i} v_{j} f_{p}(\vec{x}, t)+\delta_{i j}\left(\int d \Gamma_{p} E_{p} f_{p}(\vec{x}, t)-\mathcal{E}(\vec{x}, t)\right),
$$

Similar expressions hold in relativistic theories, see [307] and [308]. A difficulty in constructing quasi-particle models of equilibrium and non-equilibrium properties is to find an explicit expression for $\mathcal{E}\left[f_{p}\right]$. This problem can be avoided by focusing on the enthalpy

$$
\mathcal{E}+P=\int d \Gamma_{p}\left(\frac{1}{3} \vec{v} \cdot \vec{p}+E_{p}\right) f_{p}(\vec{x}, t)
$$

which can be expressed directly in terms of quasi-particle properties $E_{p}$ and $v_{p}=\nabla_{p} E_{p}$. Equation (95) can be used in connection with any of the quasiparticle theories discussed earlier in this Section in order to construct a consistent kinetic and thermodynamic model. Indeed, enthalpy functionals are also at the center of many quasi-particle models that explore the more difficult regime near the QCD phase transition, see [309, 310]. 


\section{Dilepton Production}

The measurement of dileptons, i.e. lepton anti-lepton pairs, such as $\left(e^{+} e^{-}\right)$or $\left(\mu^{+} \mu^{-}\right)$in heavy ion collisions provides insight into the early, dense phase of the system. Dileptons, which originate from the decay of time-like virtual photons, only interact electromagnetically and thus, contrary to hadrons, do not suffer from final state interaction. Compared to real photons, dileptons offer a larger kinematic range since they are not restricted to the light cone, $E=p$.

The first measurement of electron positron pairs in a heavy ion collision was carried out by the DLS collaboration at the BEVALAC, where invariant mass spectra in proton-proton, Carbon-Carbon and $\mathrm{Ca}+\mathrm{Ca}$ collisions at beam energies up to $2 A \mathrm{GeV}[311,312]$ were measured. The motivation for these measurements was to gather information about the early phase of the system. Soon thereafter it was also realized that these measurements may be sensitive to in-medium properties of pions [313]. At high energies, in connection with the search for a QGP, thermal dileptons were proposed as signature of an equilibrated plasma $[57,314,315,316,317]$.

Since vector mesons such as $\rho, \omega$, and $\phi$ have an exclusive decay channel into lepton pairs, dileptons are an excellent probe to study the in-medium properties of vector mesons, or, more generally, the in-medium spectral function of the vectorcorrelator of the strong interaction as discussed in Section 6.1. This possibility received considerable attention due to the influential paper by Brown and Rho [49], where it was conjectured that the mass of the $\rho$-meson scales with the chiral condensate $\langle\bar{\psi} \psi\rangle$, the order parameter of chiral symmetry restoration.

The thermal dilepton production rate is given by $[315,318,319,320]$

$$
\frac{d R}{d M d^{4} q}=-\frac{\alpha^{2}}{3 \pi^{2}} \frac{L\left(M^{2}\right)}{M^{2}} \operatorname{Im} \Pi_{e m, \mu}^{\mu}\left(M, q ; \mu_{B}, T\right) f^{B}(q 0 ; T)
$$

where $\alpha$ is the fine structure constant, $f^{B}\left(q_{0} ; T\right)$ the Bose-Einstein distribution function. The lepton phase space factor

$$
L(M)=\left(1+\frac{2 m_{l}^{2}}{M^{2}} \sqrt{1-\frac{4 m_{l}^{2}}{M^{2}}}\right)
$$

is unity except for small invariant masses $M \lesssim 2 m_{l}$. The retarded (electromagnetic) current-current correlator

$$
\Pi_{e m}^{\mu, n u}=i \int d^{4} x e^{i q x} \Theta\left(x^{0}\right)\left\langle\left[J_{\mathrm{em}}^{\mu}, J_{\mathrm{em}}^{\nu}\right]\right\rangle
$$

is proportional to the isospin $(0,0)$ component of the vector correlator, Eq. (72). Therefore, the dilepton rate is proportional to the time integrated, thermally 
weighted correlation function of the (chiral) conserved vector correlator $\Pi_{V}(q)$ which enters in the Weinberg sum-rules, as discussed in Section 6.1. Consequently a dilepton measurement may provide constraints on the dynamics of chiral restoration at finite density and temperature.

According to the conjecture of Brown and Rho [49], where the above correlator is saturated by a $\rho$-meson at a reduced mass, the dilepton invariant mass spectrum for high energy heavy ion collisions should exhibit more strength at masses below the $\rho / \omega$ peak. And indeed, the first measurement of dilepton invariant mass spectra by the CERES collaboration at the CERN SPS showed such an enhancement, first in $\mathrm{S}+\mathrm{Au}$ [21] and later in $\mathrm{Pb}+\mathrm{Pb}$ collisions [24]. However, as discussed in Section 6.1, the alternative view of a broadened spectral function via mixing of the $\rho$-meson with hadronic states, predominantly excited baryons [292], could also explain the first CERES data [321]. For a detailed review see [319].

The definitive resolution between these alternative explanations was later provided by the NA60 experiment, which measured di-muon invariant mass spectra of unprecedented quality $[322,323]$. This measurement clearly ruled out the originally conjectured scaling of the $\rho$-meson mass while the picture of a broadened in-medium $\rho$-meson spectral function $[321,319,324]$ prevailed. The acceptance corrected invariant mass spectrum of excess dileptons measured by the NA60 collaboration $[322,323,325]$ is shown in Fig. 22 together with theoretical calculations by various groups $[320,326,327]$. The excess spectrum is obtained by subtracting the contributions from long lived sources, such as $\eta$ and $\omega$-Dalitz decays, direct decays of $\omega$ and $\phi$, as well as open charm and Drell-Yan pairs [325]. The excellent resolution and high statistics of the NA60 experiment allowed to measure these sources directly and to remove them in a model independent fashion. In the low mass region, $M_{i n v}<0.7 \mathrm{GeV}$, the model calculations shown in Fig. 22 diverge and the result of van Hees and Rapp [320] is closest to the data (see also [328]). While all calculations shown take into account in-medium broadening of the $\rho$-meson, those of Renk and Ruppert [326] and Dusling and Zahed [327] consider only the leading order correction in density and temperature, while van Hees and Rapp re-sum the self-energy corrections and thus take higher order effects into account.

Meanwhile, low mass dilepton spectra have also been measured at much lower energies $\sqrt{s}=2.24 \mathrm{GeV}$ by the HADES collaborations [330,331,332,333] and at higher energies (up to $\sqrt{s}=200 \mathrm{GeV}$ ) by the STAR collaboration $[334,335,336]$ and the PHENIX collaboration $[337,338]$. In both cases one finds a qualitatively similar enhancement in the mass region below the $\rho$ meson. At low energies, HADES finds that the dilepton spectrum for the small system of $\mathrm{C}+\mathrm{C}$ can be explained in terms of nucleon-nucleon scattering [331], whereas the heavier system of $\mathrm{Ar}+\mathrm{KCl}$ shows a clear enhancement [333], as shown in the right panel of Fig. 23. 


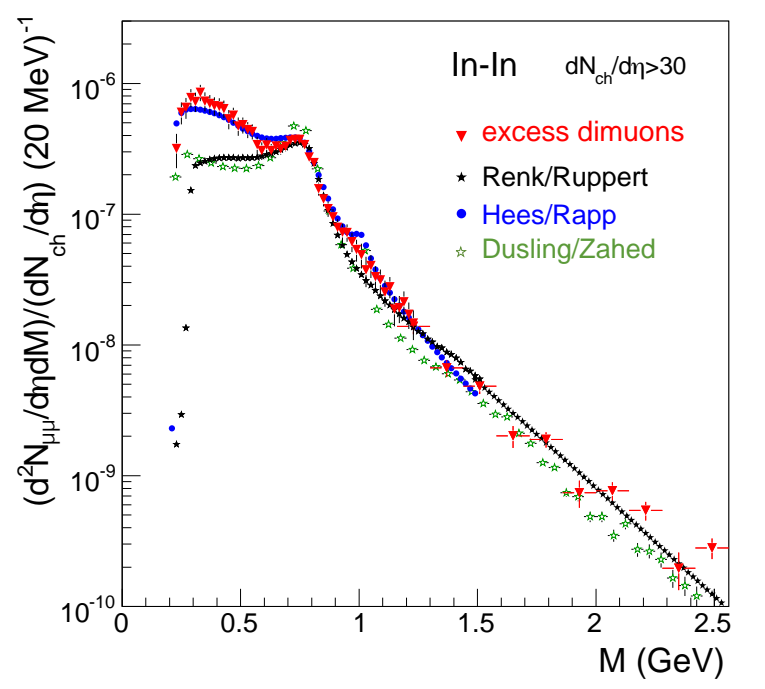

Figure 22: Invariant mass spectrum of excess dileptons from the NA60 experiment [323, 325]. Also shown are theoretical calculations by Rupert et al, [326], Rapp and van Hees [320], as well as Dusling and Zahed [327]. Figure adapted from [329].

The STAR experiment at RHIC has measured the dilepton spectra for several beam energies ranging from $\sqrt{s}=19.6 \mathrm{GeV}$ to $\sqrt{s}=200 \mathrm{GeV}$ and observes an enhancement below the $\rho$ in all cases [339]. As shown in the left panel of Fig. 23, the excess seen by STAR agrees, within the comparatively large errors, with that observed by NA60 even for the high energy collisions at $\sqrt{s}=200 \mathrm{GeV}$. One of the reasons for this mild energy dependence of the excess is that the density of hadrons hardly changes, as can be seen by the nearly constant freeze out temperature as discussed in Section 3. Furthermore, the important contribution from baryons does not depend on the net baryon density but rather on the sum of baryons and anti-baryons, which in the thermal model remains nearly constant for top SPS energies $(\sqrt{s}=17.3 \mathrm{GeV})$ and higher [340]. Below top SPS energies, on the other hand, the density of baryons and thus that of baryons and anti-baryons increases as the incoming nuclei are stopped more effectively. Consequently, one expects further enhancement of the excess below the $\rho$-mass, and indeed data taken by the CERES collaboration at $\sqrt{s}=8.75 \mathrm{GeV}$ confirm this expectation, albeit with large statistical errors [341]. Thus, the measurement of dilepton invariant mass spectra in the energy range of $5 \mathrm{GeV} \lesssim \sqrt{s} \lesssim 8 \mathrm{GeV}$ would be of great interest as this is the region of highest baryon plus anti-baryon density.

It is worth pointing out that the model of Rapp, Wambach and van Hees, is able to reproduce the observed excess for all beam energies even at the low 

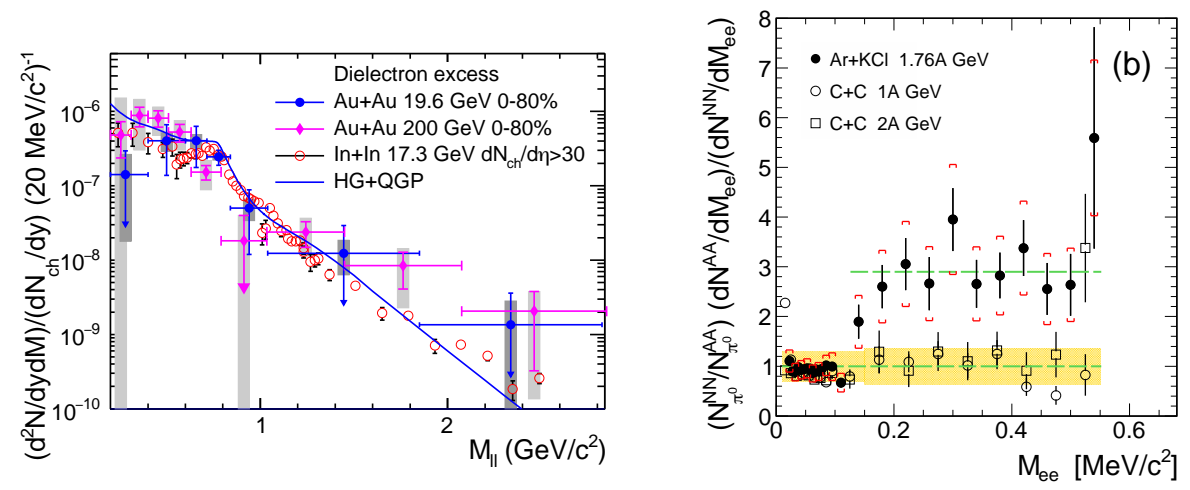

Figure 23: Left panel: Comparison of excess dileptons measured by NA60 for In + In at $\sqrt{17.3} \mathrm{GeV}$ with those by STAR for $\mathrm{Au}+\mathrm{Au}$ at $\sqrt{19.6} \mathrm{GeV}$ and $\sqrt{200} \mathrm{GeV}$. Figure adapted from [336]. Right panel: Ratio of dilepton excess for $\mathrm{Ar}+\mathrm{KCl}$ over $\mathrm{C}+\mathrm{C}$. Figure adapted from [333].

energies where the fireball consists mostly of baryon resonances [342]. In this sense it is fair to say that the question of the low mass enhancement is settled and that it is the various hadronic resonances and their interaction and mixing which are the origin of the observed excess. To which extent this can be related to the fundamental question of chiral symmetry is still an open question which, at present, can only be addressed within models [343]. Experimentally, this would require the measurement of the axial correlator. If this is feasible e.g. via $\gamma-\pi$ correlation remains to be seen.

While the low mass dilepton spectrum provides information on the chiral dynamics at finite temperature and density, the intermediate mass region between $1 \mathrm{GeV} \leq M_{\text {inv }} \lesssim 3 \mathrm{GeV}$ may provide access to the thermal radiation from the quark-gluon plasma, as argued many years ago [57, 314, 315, 316, 317]. The major sources of background in the intermediate mass region are lepton pairs from DrellYan production and semi-leptonic decays of correlated open charm pairs. The latter turns out to be especially difficult to cope with at the higher energies at RHIC and LHC, where open charm is abundantly produced. This background is less severe at SPS energies and the NA60 collaboration, which also measured open charm, could successfully remove it together with the Drell-Yan pairs. The resulting spectrum, Fig. 22, exhibits an almost perfect exponential fall off, which is consistent with thermal radiation with a temperature of $T=205 \pm 12 \mathrm{MeV}$ $[325,329]$. Since dileptons are emitted during the entire evolution of the fireball, this temperature should be considered as a lower limit of the temperature reached in these collisions. Given the thermal nature of the spectrum and the fact that lattice QCD predicts a transition temperature of $T_{c} \simeq 150 \mathrm{MeV}$ (see Section 2.3), 
one cannot but conclude that the intermediate mass dileptons observed by NA60 originate from a QGP. This argument finds support in the model calculations shown in Fig. 22, which find the spectrum above $M>1 \mathrm{GeV}$ to be dominated by thermal radiation from the QGP. This was recently confirmed in [344], where the authors augmented their calculation shown in Fig. 22 by a realistic treatment of the QGP based on the latest lattice results.

We note that the PHENIX collaboration reported a temperature of $T_{\gamma}=$ $239+-26 \mathrm{MeV}[345,346,347]$ and ALICE an even higher value of $T_{\gamma}=297 \pm$ $43 \mathrm{MeV}$ [348] from a measurement of the low transverse momentum direct photon spectrum. However, the photon momentum spectrum is subject to blue shift and, thus, the actual temperature may be considerably lower [226, 349], potentially close to the transition temperature. The dilepton invariant mass spectrum, on the other hand, is unaffected by such a blue shift, and, therefore, appears to be the preferred method to determine the temperature of the QGP.

Since the dileptons in the intermediate mass region seem to originate predominantly from the QGP, it would be desirable to measure the elliptic flow or azimuthal asymmetry of these lepton pairs. The prevailing paradigm asserts that the observed elliptic flow is generated predominantly in the QGP. If this is correct, it also should be cleanly visible with intermediate mass dileptons. To which extent such a measurement is feasible remains to be seen, and it is encouraging that first attempts to extract azimuthal asymmetries of dileptons, albeit at lower invariant mass, have successfully been carried out [350].

\section{Hadrons with heavy quarks}

We showed in Section 3 that the thermal model provides a successful description of the production of hadrons composed of light quarks in ultrarelativistic nuclear collisions. Given this result, it makes sense to ask whether a similar approach can be used in the heavy quark sector. It was realized some time ago [351] that, because of the large charm quark mass $\left(m_{c} \approx 1.2 \mathrm{GeV}\right)$, for temperatures $T$ reached realistically in a $\mathrm{Pb}-\mathrm{Pb}$ collision at LHC energy, thermal production of charm quarks is strongly suppressed compared to the number of charm quarks produced in initial, hard collisions. This clearly implies that chemical equilibration is not achieved for charm quarks and certainly not for beauty quarks. However, thermal equilibration of heavy quarks may well take place in the hot and dense fireball. Indeed, there are strong indications for significant rescattering of charm quarks after they are formed very early, less than $0.1 \mathrm{fm} / \mathrm{c}$ after the start of the collision. In the QGP, the energy loss of high energy heavy quarks is similar to that of light quarks or gluons. The measurements from the ALICE collaboration at the LHC with fully reconstructed D mesons [352] demonstrate this over a large range of transverse momenta. These measurements corroborate 
the earlier observations at RHIC, where inclusive single electron data provided first indications for energy loss of charm quarks at relatively low values of transverse momentum $[203,353]$. Further evidence for thermalization of charm quarks comes from flow studies. Measurements of elliptic flow of heavy quarks at the LHC [354] and at RHIC [353, 355] demonstrate clearly that heavy quarks also participate in the collective expansion of the hot fireball. The simultaneous description of energy loss and flow imposes further constraints on theoretical models [356].

Nearly 30 years ago, charmonium production and, in particular, the possible dissociation of charmonia in relativistic nuclear collisions was proposed as a unique signature for a dense, deconfined medium [357]. Further theoretical development considering the $\mathrm{J} / \psi$ meson and all its excited states led to the prediction of "sequential melting" at different temperatures [358, 359, 360] of charmonia with a hierarchy determined by size and binding energies of the various excited states. Measurements first at the CERN SPS and later at RHIC provided first evidence for such a dissociation mechanism, although many puzzling aspects remained. In particular, the $\mathrm{J} / \psi$ dissociation or suppression patterns observed at SPS and RHIC energies nearly coincide, although the energy density in the hot fireball is significantly increased in nuclear collisions at RHIC as compared to top SPS energy. Furthermore, the suppression measured at RHIC is smallest at mid-rapidity and suppression is stronger at forward and backward rapidities. This is opposite to what is expected in scenarios where the suppression increases with increasing energy density [361], as the energy density peaks at mid-rapidity. A recent review and compilation of data and their possible interpretation in the original Matsui-Satz dissociation scenario can be found in Ref. [362].

Lattice QCD calculations, discussed in Section 2.3, have been used to shed light on possible melting effects in the plasma (for a review see [363]). Indeed, evidence for sequential melting was found in some of these calculations. However, one should remember that lattice QCD calculations can only be used to describe completely equilibrated systems, and contain none of the dynamical effects present in a nucleus-nucleus collision at high energy. Investigating various scenarios of the possible influence of a thermal medium on charmonium production two of us proposed an entirely new approach. In this statistical hadronization model [43], the charm quarks which are all produced in initial hard collisions (see above) thermalize in the QGP. At chemical freeze-out, which for small enough values of $\mu_{b}$ (say below $100 \mathrm{MeV}$ ) closely coincides with hadronization, as discussed in Section 3, these thermalized charm quarks form hadrons with heavy quarks; their yields are determined by thermal weights equivalent to those used in the description of hadrons containing light valence quarks $[364,43]$, with an additional charm fugacity factor described below. A more detailed discussion of this new approach, together with predictions made before data from the LHC 
became available, can be found in $[365,366,126]$.

The main new feature in this statistical hadronization model is that all charmonia are formed late in the collision phase, i.e at the phase boundary (hadronization). Since the charm quarks are explicitly not in chemical equilibrium as they are produced in early, hard collisions, a charm fugacity factor $\mathrm{g}_{c}$ has to be introduced into the thermal formulation, as described in [43, 367, 368]. As a consequence, all yields for open charm hadrons are multiplied with $\mathrm{g}_{c}$ while charmonia are enhanced by a factor $\mathrm{g}_{c}^{2}$. Since $\mathrm{g}_{c}$ is (nearly) proportional to $\mathrm{N}_{c}$, the number of charm quarks, this implies a strong enhancement of charmonia with increasing collision energy. This $\mathrm{N}_{c}^{2}$ dependence implies that a charm quark in the fireball may combine with any anti-charm quark to form a $\mathrm{J} / \psi$ or other charmonium state, as long as the charm and anti-charm quarks can be causally connected. For this reason, in practice, only charm quarks within a rapidity interval of $\Delta y<1$ are considered for charmonium formation [364]. This also implies that observation of $\mathrm{J} / \psi$ production enhanced according to $\mathrm{g}_{c}^{2}$ is a clear sign for the presence of deconfined charm quarks. In view of all the above considerations, little suppression or even enhancement of charmonia was predicted for LHC energy, see, e.g., $[43,368,365]$.

Shortly after the proposal of [43], an alternative idea for production of charmonia was put forward via the kinetic recombination of charm and anti-charm quarks in the QGP [44]. In this approach which has been taken up by several groups $[369,370,371,372]$ continuous dissociation and generation of charmonia is modeled by rate equations and takes place during the lifetime of the QGP. Also in this approach, the final yield of charmonia scales $\propto N_{c}^{2}$ and charmonium enhancement is expected as $\mathrm{N}_{c}$ becomes very large.

Before describing the recent data from the LHC we note that, for historical reasons, both approaches are dubbed somewhat colloquially in the literature as "charmonium regeneration" models.

Since the center of mass energy increase between RHIC and LHC is more than a factor of 20 , the corresponding increase in $\mathrm{N}_{c}$ was estimated to be about an order of magnitude, while the energy density of the fireball should increase by about a factor of 2-3. Consequently, the measurement of the centrality dependence of $R_{\mathrm{AA}}$ for inclusive $\mathrm{J} / \psi$ production in $\mathrm{Pb}-\mathrm{Pb}$ collisions at the $\mathrm{LHC}$ was expected to provide important information on the question of strong suppression due to Debye screening vs. regeneration.

Indeed, the first data from the LHC on the centrality dependence of $p_{T}$ integrated $\mathrm{J} / \psi$ yields [375] exhibited, for forward rapidities, values of the nuclear modification factor $R_{\mathrm{AA}}$ which significantly exceeded those measured at RHIC energies, providing first qualitative evidence for the charmonium regeneration scenario. Soon higher statistics data including measurements at mid-rapidity [42] confirmed this, see Fig. 24. Note that the quantity on the horizontal axis on 

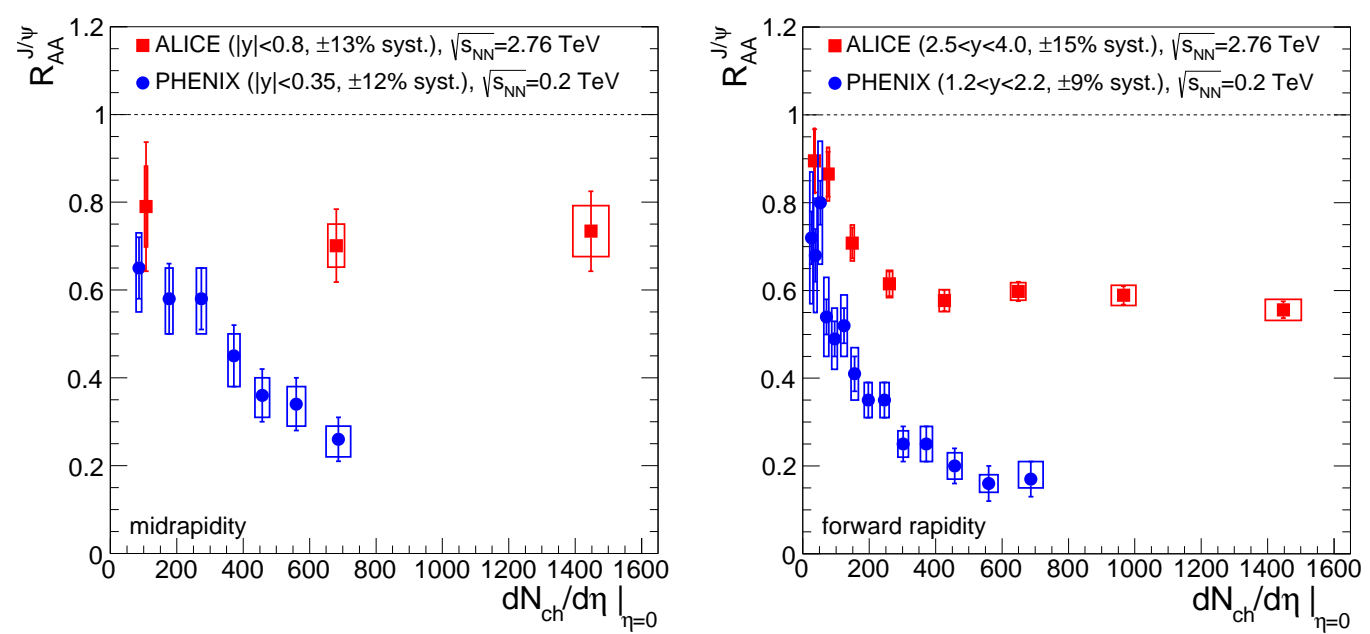

Figure 24: Dependence of the nuclear modification factor $R_{\mathrm{AA}}$ for inclusive $\mathrm{J} / \psi$ production on the multiplicity density (at $\eta=0$ ). The left panel shows data at mid-rapidity, the right panel at forward rapidity. The data are integrated over $p_{T}$ and are from the PHENIX collaboration [373] at RHIC and the ALICE collaboration [42] at the LHC. Figure taken from [42].

this plot, the charged particle pseudo-rapidity density, is essentially proportional to the energy density. For the most central collisions, the energy density between RHIC and LHC increases by more than a factor of 2 , but $R_{\mathrm{AA}}$ does not decrease at the higher energy density, but rather increases by nearly a factor of 3 .

Further evidence for the regeneration mechanism comes from the fact that the data are well described by both the statistical hadronization model [126] and by transport models [369, 370]. This is demonstrated in Fig. 25, although admittedly the uncertainties in the models are still quite large. The main uncertainty is due to the fact that the charm production cross section has not yet been measured for $\mathrm{Pb}-\mathrm{Pb}$ collisions at the LHC and, when extrapolating from the pp cross section the uncertainty in the nuclear parton distributions is significant.

Recent data also provided insight into the transverse momentum dependence of charmonium production at the LHC as illustrated by the $p_{T}$ dependence of $R_{\mathrm{AA}}$ measured by ALICE and CMS, shown in Fig. 26. These data are quite well described in the above discussed transport models [370, 372]. There, at small transverse momenta a large fraction of the $\mathrm{J} / \psi$ yield in $\mathrm{Pb}-\mathrm{Pb}$ collisions comes from the regeneration mechanism, while this fraction decreases with increasing $p_{T}$. Within the transport model description, regeneration is mainly a low- $p_{T}$ phenomenons and this can account well for the data.

On the other hand, an important assumption underlying the regeneration process is that the charm quarks reach, during the evolution of the collision, some degree of equilibration. Inspection of the centrality dependent shape of 


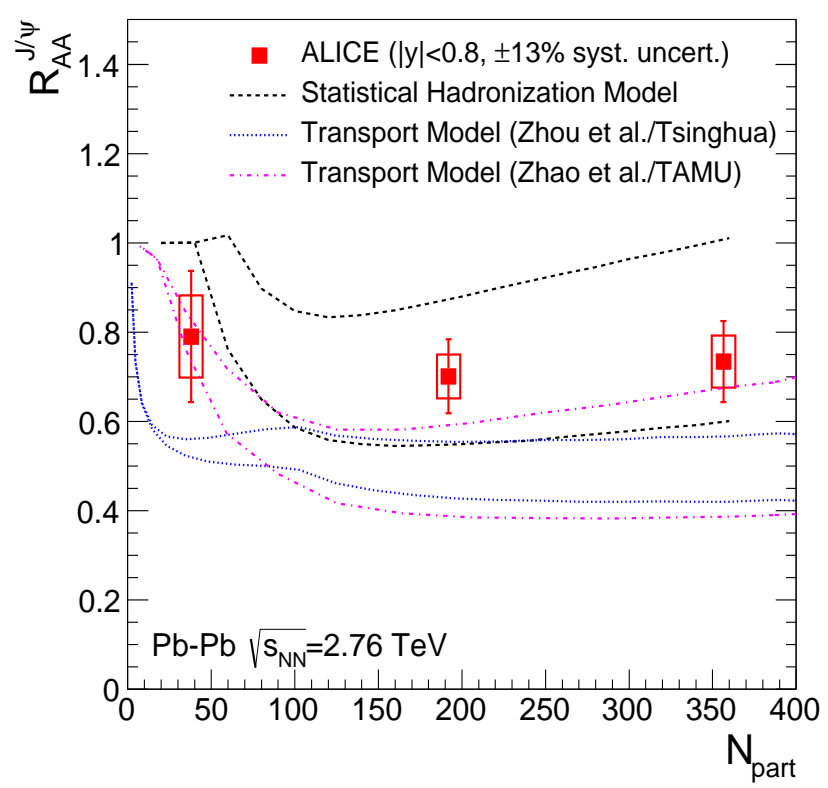

Figure 25: Centrality dependence of the nuclear modification factor for inclusive $\mathrm{J} / \psi$ production at the LHC. The data at mid-rapidity [42] are compared to model calculations: the statistical hadronization model [126] and transport models of the TAMU [370, 374] and Tsinghua [369, 372] groups. The band of the statistical hadronization model reflects the uncertainty in the charm production cross section: The lower and upper black dashed lines correspond to $\mathrm{d} \sigma_{c \bar{c}} / \mathrm{dy}=0.3$ and $0.4 \mathrm{mb}$. Figure taken from [42].

transverse momentum spectra [42], quantified by the mean and variance of the distributions, lends support to this assumption. Furthermore, the measurement of a clear signal of $\mathrm{J} / \psi$ elliptic flow at the LHC [376] brings an additional argument towards charm quark thermalization. The $\mathrm{J} / \psi$ data at RHIC are compatible with a vanishing flow signal [377], but the errors are large enough to also allow the magnitude of the flow signal observed at LHC energy. A non-zero $v_{2}$ signal was also measured for $\mathrm{J} / \psi$ production at the SPS [378] but was interpreted as a path-length dependence of Debye screening.

The data by the CMS and ALICE collaborations measured at higher $p_{T}$ $[379,42]$ exhibit a strong suppression of $\mathrm{J} / \psi$ mesons in $\mathrm{Pb}-\mathrm{Pb}$ compared to pp collisions. Importantly, this suppression is similar in magnitude to that measured for open-charm hadrons. This suppression in the $\mathrm{J} / \psi$ signal therefore may also be a result of the energy loss of high- $p_{T}$ heavy quarks in the hot and dense fireball, leading to thermalization and, finally, at hadronization, to the formation of charmonia and open charm mesons.

Based on the new LHC data and the above observations and considerations, charmonium production should be considered a probe of deconfinement of heavy 


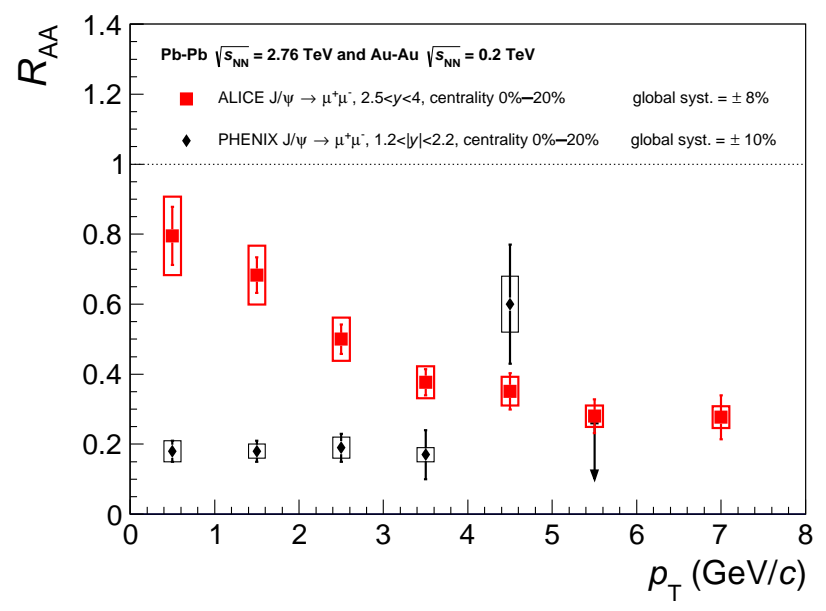

Figure 26: Transverse momentum dependence of the nuclear modification factor for $\mathrm{J} / \psi$ production measured at RHIC [373] and at the LHC [42]. Figure taken from [42].

quarks rather than a "thermometer" of the QGP. Within the framework of the thermal approach sketched here, the charmonium states become important probes of the phase boundary between the deconfined and the hadron phase. The quarkonium data at the LHC have found a natural explanation in terms of the regeneration model while no other plausible interpretation was put forward.

Discriminating between the two pictures of disintegration and regeneration of charmonia in the QGP versus that of assembly of charmonia from deconfined charm quarks at the phase boundary (statistical hadronization) will shed light on fundamental questions connected with the fate of bound states in a deconfined medium: Can a $\mathrm{J} / \psi$ meson be formed from deconfined charm quarks at temperatures well above the deconfinement phase transition [366]? For further discussions about bound states in a hot medium see also [380, 381, 382]. Data at the top LHC energy, including measurements on $\psi(2 S)$ production in $\mathrm{Pb}-\mathrm{Pb}$ collisions, should clarify these questions. A subject of intense current research is the study of $\psi(2 S)$ production in light systems such as d-Au collisions at RHIC [383] and in $\mathrm{p}-\mathrm{Pb}$ at the LHC [384], where unexpected findings of suppression indicate possible final-state effects.

We close this Section with a short discussion of LHC measurements on the production of bottomonia (mesons composed of $b \bar{b}$ quarks) [385, 386, 387] and of similar measurements at RHIC [388]. The nuclear modification factor for the $\Upsilon$ states at both RHIC and LHC clearly shows a suppression pattern [385, 388] with increasing suppression from $\Upsilon(1)$ to $\Upsilon(2 s)$ and $\Upsilon(3 s)$. The CMS data are displayed in Fig. 27. Whether this indicates sequential suppression due to different binding energies or radii of the quarkonia is currently the subject of an 

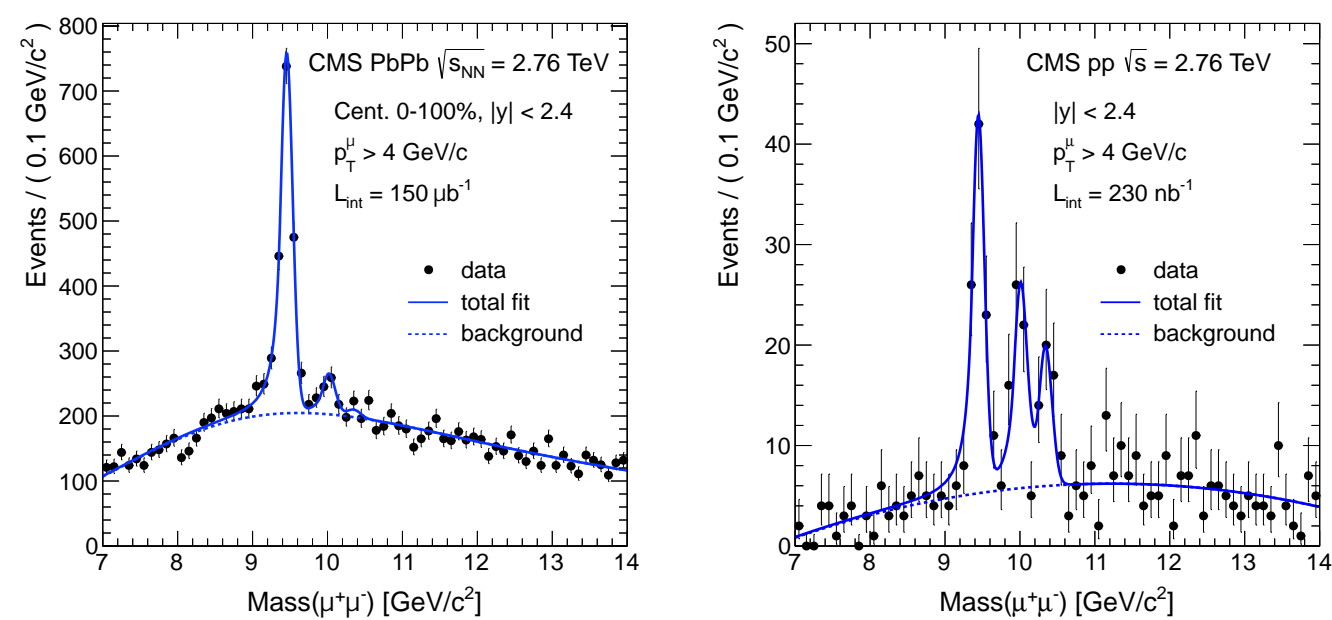

Figure 27: Suppression pattern of $\Upsilon$ states measured by the CMS collaboration in $\mathrm{Pb}-\mathrm{Pb}$ collisions (left) and pp collisions (right). Figure taken from [385].

intense debate. We first note that the radii and binding energies of $\mathrm{J} / \psi$ and $\Upsilon(2 s)$ mesons are similar, but the observed suppression is very different, casting doubt on a simple Debye screening interpretation. Further there are the questions of possible thermal equilibration of beauty quarks as well as on the magnitude of feeding from higher-lying bottomonia into the 1s state and on the issue of the rapidity dependence of the nuclear modification factor of bottomonia. Together, the data from CMS [385] and from ALICE [387] indicate that $R_{\mathrm{AA}}$ peaks at midrapidity although the energy density is largest there. This is a real challenge to various theoretical models. The situation is succinctly described in [389].

An interesting observation is made in [390]: the ratio $\Upsilon(2 S) / \Upsilon(1 S)$, is characteristically different in $\mathrm{Pb}-\mathrm{Pb}$ compared to pp collisions. Indeed, the data are consistent, for the most central $\mathrm{Pb}-\mathrm{Pb}$ collisions, with the value predicted within the framework of the statistical hadronization model for a temperature $T=159$ $\mathrm{MeV}$, very close to what was used for the charmonium case. Is the bottomonium situation also described in the regeneration picture? More precise data, on the beauty cross section in $\mathrm{Pb}-\mathrm{Pb}$ collisions, the question of possible thermalization of b-quarks, but also in particular for bottomonia on the dependence of $R_{\mathrm{AA}}$ on transverse momentum and rapidity, are needed to clarify this interesting possibility.

\section{Outlook}

The relativistic heavy ion programs at RHIC and the LHC have led to significant advances in our understanding of hot and dense matter. The experiments 
have demonstrated that rapid local equilibration takes place, and this discovery has enabled a systematic program of determining the properties of strongly interacting matter, such as the equation of state, the shear and bulk viscosity, the heavy quark diffusion constant. In addition, it has been established that even very high energy (several hundreds of $\mathrm{GeV}$ ) partons lose large fractions of their energy in the hot fireball, and first signs of deconfinement have emerged from comparative studies of charmonium production at RHIC and the LHC.

In the near future, energy and luminosity upgrades at the LHC, and a suite of detector upgrades at LHC and RHIC, will allow much more precise measurements of these and related quantities, and explore the limits of the perfect fluid paradigm in terms of system size, geometry, and beam energy. In addition to that, experiments at the ongoing beam energy scan program at RHIC are being performed to search for critical fluctuations, with the goal of identifying a possible endpoint of the QCD phase transition. Furthermore, searches will continue for exotic phenomena such as the existence of exotic bound states as e.g. dibaryons consisting of a multi-strange baryon with a nucleon, or of a $\Lambda$ hyperon with two neutrons. Other opportunities for discovery include the search for quantum anomalies in the quark-gluon fluid at RHIC and LHC.

In the longer term a number of facilities are expected to come online that will study the regime of maximum baryon density, which is reached at energies of a few $\mathrm{GeV}$ per baryon in the center of mass. This is the regime previously explored in the AGS and the SPS fixed target programs. The new set of experiments planned at the FAIR facility at GSI, NICA at Dubna, and JPARC, will study collisions in this energy regime with unprecedented precision, and will make use of all the knowledge that was gained at the higher energies. The regime of maximum baryon density presents new challenges: The applicability of fluid dynamics is not clear, the freeze-out line no longer follows the phase boundary, and modifications of hadron properties are likely to be important. Furthermore, the 'nearly instantaneous' collision time scenario which at collider energies makes the time sequence of events in a collision transparent will have to be replaced by collision times comparable to the lifetime of the hot fireball, a challenge for all theoretical descriptions. Nevertheless, great opportunities exist and are bounded only by our own ingenuity.

Gerry Brown's unique style and insights will continue to guide our work in future research, and that of many others in the field who were fortunate to interact with him over the course of his long career.

\section{Acknowledgements}

The authors thank Jiangyong Jia, Klaus Reygers, Hans Specht, Joachim Stroth, and Zhangbu Xu for helpful communications. We also thank Anton An- 
dronic and Krzysztof Redlich for a long-standing collaboration on issues related to the thermal/statistical model. V.K. and T.S. are supported by the Director, Office of Energy Research, Office of High Energy and Nuclear Physics, Divisions of Nuclear Physics, of the U.S. Department of Energy under Contract No. DEAC02-05CH11231 and DE-FG02-03ER41260, respectively. J.S. is supported in part by the German BMBF ministry and by the ExtreMe Matter Institute EMMI.

[1] H. Fritzsch, M. Gell-Mann, H. Leutwyler, Advantages of the Color Octet Gluon Picture, Phys. Lett. B47 (1973) 365-368. doi:10.1016/0370-2693(73) 90625-4.

[2] D. J. Gross, F. Wilczek, Ultraviolet Behavior of Nonabelian Gauge Theories, Phys.Rev.Lett. 30 (1973) 1343-1346. doi:10.1103/PhysRevLett.30.1343.

[3] H. D. Politzer, Reliable Perturbative Results for Strong Interactions?, Phys.Rev.Lett. 30 (1973) 1346-1349. doi:10.1103/PhysRevLett.30.1346.

[4] J. C. Collins, M. J. Perry, Superdense matter: Neutrons or asymptotically free quarks?, Phys. Rev. Lett. 34 (1975) 1353.

[5] N. Cabibbo, G. Parisi, Exponential Hadronic Spectrum and Quark Liberation, Phys. Lett. B59 (1975) 67-69. doi:10.1016/0370-2693(75)90158-6.

[6] E. V. Shuryak, Theory of Hadronic Plasma, Sov. Phys. JETP 47 (1978) 212-219, [Zh. Eksp. Teor. Fiz.74,408(1978)].

[7] R. Hagedorn, Statistical thermodynamics of strong interactions at high-energies, Nuovo Cim. Suppl. 3 (1965) 147-186.

[8] M. Creutz, Monte Carlo Study of Quantized SU(2) Gauge Theory, Phys. Rev. D21 (1980) 2308-2315. doi:10.1103/PhysRevD.21.2308.

[9] M. Creutz, Confinement and the Critical Dimensionality of Space-Time, Phys. Rev. Lett. 43 (1979) 553-556, [Erratum: Phys. Rev. Lett.43,890(1979)]. doi:10.1103/PhysRevLett. 43.553.

[10] S. A. Chin, Transition to Hot Quark Matter in Relativistic Heavy Ion Collision, Phys. Lett. B78 (1978) 552-555. doi:10.1016/0370-2693(78)90637-8.

[11] E. Fermi, High-energy nuclear events, Prog. Theor. Phys. 5 (1950) 570-583. doi:10.1143/ PTP. 5.570.

[12] L. D. Landau, On the multiparticle production in high-energy collisions, Izv. Akad. Nauk Ser. Fiz. 17 (1953) 51-64.

[13] G. Baym, RHIC: From dreams to beams in two decades, Nucl. Phys. A698 (2002) XXIIIXXXII. arXiv:hep-ph/0104138, doi:10.1016/S0375-9474(01)01342-2.

[14] Report of the workshop on $\mathrm{GeV} /$ nucleon collisions of heavy ions: how and why, November 29-December 1, 1974, Bear Mountain, New York. Report BNL-50445; CONF-741152.

[15] Proceedings: Workshop on Ultra-Relativistic Nuclear Collisions, 1st, Berkeley, Calif., May 21-24, 1979. Report LBL-8957, CONF-7905107.

[16] R. Stock, A. M. Poskanzer, Heavy Ion Reactions at Relativistic Energies, Comments Nucl. Part. Phys. 7 (2) (1977) 41-47.

[17] S. Nagamiya, J. Randrup, T. J. M. Symons, Nuclear collisions at high energies, Ann. Rev. Nucl. Part. Sci. 34 (1984) 155-187. doi:10.1146/annurev.ns.34.120184.001103.

[18] H. A. Gustafsson, et al., Collective Flow Observed in Relativistic Nuclear Collisions, Phys. Rev. Lett. 52 (1984) 1590-1593. doi:10.1103/PhysRevLett.52.1590.

[19] P. Braun-Munzinger, J. Stachel, J. P. Wessels, N. Xu, Thermal equilibration and expansion in nucleus-nucleus collisions at the AGS, Phys. Lett. B344 (1995) 43-48. arXiv:nucl-th/ 9410026, doi:10.1016/0370-2693(94)01534-J.

[20] P. Braun-Munzinger, J. Stachel, J. P. Wessels, N. Xu, Thermal and hadrochemical equilibration in nucleus-nucleus collisions at the SPS, Phys. Lett. B365 (1996) 1-6. arXiv:nucl-th/9508020, doi:10.1016/0370-2693(95)01258-3. 
[21] G. Agakichiev, et al., Enhanced production of low mass electron pairs in $200-\mathrm{GeV} / \mathrm{u} \mathrm{S}$ - Au collisions at the CERN SPS, Phys. Rev. Lett. 75 (1995) 1272-1275. doi:10.1103/ PhysRevLett.75.1272.

[22] M. C. Abreu, et al., Observation of a threshold effect in the anomalous $J / \psi$ suppression, Phys. Lett. B450 (1999) 456-466. doi:10.1016/S0370-2693(99)00178-1.

[23] E. Andersen, et al., Strangeness enhancement at mid-rapidity in $\mathrm{Pb} \mathrm{Pb}$ collisions at 158 A-GeV/c, Phys. Lett. B449 (1999) 401-406. doi:10.1016/S0370-2693(99) 00140-9.

[24] G. Agakishiev, et al., Low mass e+ e- pair production in $158 / \mathrm{A}-\mathrm{GeV} \mathrm{Pb}-\mathrm{Au}$ collisions at the CERN SPS, its dependence on multiplicity and transverse momentum, Phys. Lett. B422 (1998) 405-412. arXiv:nucl-ex/9712008, doi:10.1016/S0370-2693(98)00083-5.

[25] K. H. Ackermann, et al., Elliptic flow in $\mathrm{Au}+\mathrm{Au}$ collisions at $s_{N N}^{1 / 2}=130-\mathrm{GeV}$, Phys. Rev. Lett. 86 (2001) 402-407, first paper on ellitpic flow. arXiv:nucl-ex/0009011, doi: 10.1103/PhysRevLett.86.402.

[26] K. Adcox, et al., Suppression of hadrons with large transverse momentum in central $\mathrm{Au}+\mathrm{Au}$ collisions at $\sqrt{s_{N N}}=130-\mathrm{GeV}$, Phys. Rev. Lett. 88 (2002) 022301. arXiv: nucl-ex/0109003, doi:10.1103/PhysRevLett.88.022301.

[27] B. B. Back, et al., The PHOBOS perspective on discoveries at RHIC, Nucl. Phys. A757 (2005) 28-101. arXiv:nucl-ex/0410022, doi:10.1016/j.nuclphysa.2005.03.084.

[28] I. Arsene, et al., Quark gluon plasma and color glass condensate at RHIC? The Perspective from the BRAHMS experiment, Nucl. Phys. A757 (2005) 1-27. arXiv:nucl-ex/0410020, doi:10.1016/j.nuclphysa.2005.02.130.

[29] K. Adcox, et al., Formation of dense partonic matter in relativistic nucleus-nucleus collisions at RHIC: Experimental evaluation by the PHENIX collaboration, Nucl. Phys. A757 (2005) 184-283. arXiv:nucl-ex/0410003, doi:10.1016/j.nuclphysa.2005.03.086.

[30] J. Adams, et al., Experimental and theoretical challenges in the search for the quark gluon plasma: The STAR Collaboration's critical assessment of the evidence from RHIC collisions, Nucl. Phys. A757 (2005) 102-183. arXiv:nucl-ex/0501009, doi:10.1016/j. nuclphysa.2005.03.085.

[31] J. M. Maldacena, The Large N limit of superconformal field theories and supergravity, Int. J. Theor. Phys. 38 (1999) 1113-1133, [Adv. Theor. Math. Phys.2,231(1998)]. arXiv: hep-th/9711200, doi:10.1023/A:1026654312961.

[32] G. Policastro, D. Son, A. Starinets, The Shear viscosity of strongly coupled N=4 supersymmetric Yang-Mills plasma, Phys.Rev.Lett. 87 (2001) 081601. arXiv:hep-th/0104066, doi:10.1103/PhysRevLett.87.081601.

[33] K. Dusling, D. Teaney, Simulating elliptic flow with viscous hydrodynamics, Phys. Rev. C77 (2008) 034905. arXiv:0710.5932, doi:10.1103/PhysRevC.77.034905.

[34] P. Romatschke, U. Romatschke, Viscosity Information from Relativistic Nuclear Collisions: How Perfect is the Fluid Observed at RHIC?, Phys. Rev. Lett. 99 (2007) 172301. arXiv:0706.1522, doi:10.1103/PhysRevLett.99.172301.

[35] Y. Aoki, G. Endrodi, Z. Fodor, S. D. Katz, K. K. Szabo, The order of the quantum chromodynamics transition predicted by the standard model of particle physics, Nature 443 (2006) 675-678. arXiv: hep-lat/0611014, doi:10.1038/nature05120.

[36] Y. Aoki, Z. Fodor, S. D. Katz, K. K. Szabo, The QCD transition temperature: Results with physical masses in the continuum limit, Phys. Lett. B643 (2006) 46-54. arXiv: hep-lat/0609068, doi:10.1016/j.physletb.2006.10.021.

[37] Y. Aoki, S. Borsanyi, S. Dür, Z. Fodor, S. D. Katz, S. Krieg, K. K. Szabo, The QCD transition temperature: results with physical masses in the continuum limit II., JHEP 06 (2009) 088. arXiv:0903.4155, doi:10.1088/1126-6708/2009/06/088.

[38] A. Bazavov, T. Bhattacharya, M. Cheng, C. DeTar, H. Ding, F. Karsch, et al., The chiral and deconfinement aspects of the QCD transition, Phys.Rev. D85 (2012) 054503. 
arXiv:1111.1710, doi:10.1103/PhysRevD.85.054503.

[39] A. Bazavov, et al., Equation of state in ( 2+1 )-flavor QCD, Phys. Rev. D90 (9) (2014) 094503. arXiv:1407.6387, doi:10.1103/PhysRevD.90.094503.

[40] B. Abelev, et al., Long-range angular correlations on the near and away side in $\mathrm{p}-\mathrm{Pb}$ collisions at $\sqrt{s_{\mathrm{NN}}}=5.02 \mathrm{TeV}$, Phys. Lett. B 719 (2013) 29-41. arXiv:1212.2001, doi: $10.1016 / j$. physletb. 2013.01.012.

[41] V. Khachatryan, et al., Evidence for Collective Multiparticle Correlations in p- $\mathrm{Pb}$ Collisions, Phys. Rev. Lett. 115 (1) (2015) 012301. arXiv:1502.05382, doi:10.1103/ PhysRevLett.115.012301.

[42] B. B. Abelev, et al., Centrality, rapidity and transverse momentum dependence of $J / \psi$ suppression in $\mathrm{Pb}-\mathrm{Pb}$ collisions at $\sqrt{s_{\mathrm{NN}}}=2.76 \mathrm{TeV}$, Phys. Lett. B734 (2014) 314-327. arXiv: 1311.0214, doi:10.1016/j.physletb.2014.05.064.

[43] P. Braun-Munzinger, J. Stachel, (Non)thermal aspects of charmonium production and a new look at $J / \psi$ suppression, Phys. Lett. B490 (2000) 196-202. arXiv:nucl-th/0007059, doi:10.1016/S0370-2693(00)00991-6.

[44] R. L. Thews, M. Schroedter, J. Rafelski, Enhanced $J / \psi$ production in deconfined quark matter, Phys. Rev. C63 (2001) 054905. arXiv:hep-ph/0007323, doi:10.1103/PhysRevC. 63.054905.

[45] G. Odyniec, RHIC Beam Energy Scan Program: Phase I and II, PoS CPOD2013 (2013) 043.

[46] T. L. Ainsworth, E. Baron, G. E. Brown, J. Cooperstein, M. Prakash, Equation of State of Dense Nuclear Matter, Nucl. Phys. A464 (1987) 740-768. doi:10.1016/0375-9474(87) 90373-3.

[47] G. E. Brown, Heavy ion collisions at Bevalac energies, Comments Nucl. Part. Phys. A19 (1990) 185-208.

[48] G. Bertsch, B. Li, G. Brown, V. Koch, Pion Collectivity in Relativistic Heavy Ion Collisions, Nucl.Phys. A490 (1988) 745-755. doi:10.1016/0375-9474(88)90024-3.

[49] G. E. Brown, M. Rho, Scaling effective Lagrangians in a dense medium, Phys. Rev. Lett. 66 (1991) 2720-2723. doi:10.1103/PhysRevLett.66.2720.

[50] G. E. Brown, A. D. Jackson, H. A. Bethe, P. M. Pizzochero, The Hadron to quark gluon transition in relativistic heavy ion collisions, Nucl. Phys. A560 (1993) 1035-1074. doi:10.1016/0375-9474(93) 90145-N.

[51] G. E. Brown, J. Stachel, G. M. Welke, Pions from resonance decay in Brookhaven relativistic heavy ion collisions, Phys. Lett. B253 (1991) 19-22. doi:10.1016/0370-2693(91) 91356-Z.

[52] S. R. Coleman, E. J. Weinberg, Radiative Corrections as the Origin of Spontaneous Symmetry Breaking, Phys.Rev. D7 (1973) 1888-1910. doi:10.1103/PhysRevD.7.1888.

[53] K. Nakamura, et al., Review of particle physics, J. Phys. G37 (2010) 075021. doi:10. 1088/0954-3899/37/7A/075021.

[54] M. Gell-Mann, R. J. Oakes, B. Renner, Behavior of current divergences under SU(3) x SU(3), Phys. Rev. 175 (1968) 2195-2199. doi:10.1103/PhysRev.175.2195.

[55] S. R. Coleman, E. Witten, Chiral Symmetry Breakdown in Large N Chromodynamics, Phys. Rev. Lett. 45 (1980) 100. doi:10.1103/PhysRevLett.45.100.

[56] G. 't Hooft, Naturalness, chiral symmetry, and spontaneous chiral symmetry breaking, NATO Sci. Ser. B 59 (1980) 135.

[57] E. V. Shuryak, Quark-Gluon Plasma and Hadronic Production of Leptons, Photons and Psions, Phys. Lett. B78 (1978) 150, [Yad. Fiz.28,796(1978)]. doi:10.1016/0370-2693(78) 90370-2.

[58] A. D. Linde, Infrared Problem in Thermodynamics of the Yang-Mills Gas, Phys. Lett. B96 (1980) 289. doi:10.1016/0370-2693(80)90769-8.

[59] R. D. Pisarski, F. Wilczek, Remarks on the Chiral Phase Transition in Chromodynamics, 
Phys. Rev. D29 (1984) 338-341. doi:10.1103/PhysRevD.29.338.

[60] M. A. Stephanov, QCD phase diagram and the critical point, Prog. Theor. Phys. Suppl. 153 (2004) 139-156. arXiv: hep-ph/0402115.

[61] Z. Fodor, S. D. Katz, Lattice determination of the critical point of qcd at finite $\mathrm{t}$ and $\mathrm{mu}$, JHEP 03 (2002) 014. arXiv:hep-lat/0106002.

[62] C. Allton, S. Ejiri, S. Hands, O. Kaczmarek, F. Karsch, et al., The QCD thermal phase transition in the presence of a small chemical potential, Phys.Rev. D66 (2002) 074507. arXiv:hep-lat/0204010, doi:10.1103/PhysRevD.66.074507.

[63] F. Karsch, C. R. Allton, S. Ejiri, S. J. Hands, O. Kaczmarek, E. Laermann, C. Schmidt, Where is the chiral critical point in three flavor QCD?, Nucl. Phys. Proc. Suppl. 129 (2004) 614-616, [,614(2003)]. arXiv:hep-lat/0309116, doi:10.1016/S0920-5632(03)02659-8.

[64] Z. Fodor, S. Katz, Critical point of QCD at finite $\mathrm{T}$ and $\mathrm{mu}$, lattice results for physical quark masses, JHEP 0404 (2004) 050. arXiv:hep-lat/0402006, doi:10.1088/ 1126-6708/2004/04/050.

[65] R. V. Gavai, S. Gupta, QCD at finite chemical potential with six time slices, Phys. Rev. D78 (2008) 114503. arXiv:0806.2233, doi:10.1103/PhysRevD.78.114503.

[66] S. Datta, R. V. Gavai, S. Gupta, The QCD Critical Point : marching towards continuum, Nucl. Phys. A904-905 (2013) 883c-886c. arXiv:1210.6784, doi:10.1016/j.nuclphysa. 2013.02.156.

[67] P. de Forcrand, O. Philipsen, Constraining the QCD phase diagram by tricritical lines at imaginary chemical potential, Phys. Rev. Lett. 105 (2010) 152001. arXiv:1004.3144, doi:10.1103/PhysRevLett.105.152001.

[68] M. A. Stephanov, K. Rajagopal, E. V. Shuryak, Signatures of the tricritical point in QCD, Phys. Rev. Lett. 81 (1998) 4816-4819. arXiv:hep-ph/9806219.

[69] J. Pochodzalla, et al., Probing the nuclear liquid - gas phase transition, Phys. Rev. Lett. 75 (1995) 1040-1043. doi:10.1103/PhysRevLett.75.1040.

[70] J. B. Elliott, P. T. Lake, L. G. Moretto, L. Phair, Determination of the coexistence curve, critical temperature, density, and pressure of bulk nuclear matter from fragment emission data, Phys. Rev. C87 (5) (2013) 054622. doi:10.1103/PhysRevC.87.054622.

[71] G. 't Hooft, A Planar Diagram Theory for Strong Interactions, Nucl. Phys. B72 (1974) 461. doi:10.1016/0550-3213(74)90154-0.

[72] E. Witten, Baryons in the 1/n Expansion, Nucl. Phys. B160 (1979) 57. doi:10.1016/ 0550-3213 (79) 90232-3.

[73] T. D. Cohen, QCD strings and the thermodynamics of the metastable phase of QCD at large N(c), Phys. Lett. B637 (2006) 81-84. arXiv:hep-th/0602037, doi:10.1016/j . physletb.2006.04.035.

[74] B. Lucini, M. Teper, U. Wenger, Properties of the deconfining phase transition in SU(N) gauge theories, JHEP 02 (2005) 033. arXiv:hep-lat/0502003, doi:10.1088/1126-6708/ 2005/02/033.

[75] M. G. Alford, K. Rajagopal, F. Wilczek, Color flavor locking and chiral symmetry breaking in high density QCD, Nucl. Phys. B537 (1999) 443-458. arXiv:hep-ph/9804403, doi: 10.1016/S0550-3213(98)00668-3.

[76] T. Schäfer, Patterns of symmetry breaking in QCD at high baryon density, Nucl. Phys. B575 (2000) 269-284. arXiv:hep-ph/9909574, doi:10.1016/S0550-3213(00)00063-8.

[77] M. G. Alford, A. Schmitt, K. Rajagopal, T. Schäfer, Color superconductivity in dense quark matter, Rev. Mod. Phys. 80 (2008) 1455-1515. arXiv:0709.4635, doi:10.1103/ RevModPhys.80.1455.

[78] T. Schäfer, F. Wilczek, Continuity of quark and hadron matter, Phys. Rev. Lett. 82 (1999) 3956-3959. arXiv:hep-ph/9811473, doi:10.1103/PhysRevLett.82.3956.

[79] T. Hatsuda, M. Tachibana, N. Yamamoto, G. Baym, New critical point induced by the axial anomaly in dense QCD, Phys. Rev. Lett. 97 (2006) 122001. arXiv:hep-ph/0605018, 
doi:10.1103/PhysRevLett.97.122001.

[80] J. I. Kapusta, Quantum Chromodynamics at High Temperature, Nucl. Phys. B148 (1979) 461-498. doi:10.1016/0550-3213(79) 90146-9.

[81] P. B. Arnold, C. Dogan, G. D. Moore, The Bulk Viscosity of High-Temperature QCD Phys. Rev. D74 (2006) 085021. arXiv:hep-ph/0608012, doi:10.1103/PhysRevD.74. 085021.

[82] P. Bedaque, A. W. Steiner, Sound velocity bound and neutron stars, Phys. Rev. Lett. 114 (3) (2015) 031103. arXiv:1408.5116, doi:10.1103/PhysRevLett.114.031103.

[83] K. Kajantie, M. Laine, K. Rummukainen, Y. Schroder, The Pressure of hot QCD up to g6 $\ln (1 / \mathrm{g})$, Phys. Rev. D67 (2003) 105008. arXiv:hep-ph/0211321, doi:10.1103/PhysRevD. 67.105008.

[84] J.-P. Blaizot, E. Iancu, A. Rebhan, Thermodynamics of the high temperature quark gluon plasma, in: In *Hwa, R.C. (ed.) et al.: Quark gluon plasma* 60-122, 2003. arXiv: hep-ph/0303185.

URL http://link.aip.org/link/?APC/739/63

[85] S. Borsanyi, G. Endrodi, Z. Fodor, A. Jakovac, S. D. Katz, et al., The QCD equation of state with dynamical quarks, JHEP 1011 (2010) 077. arXiv:1007.2580, doi:10.1007/ JHEP11 (2010) 077.

[86] Z. Fodor, S. D. Katz, The Phase diagram of quantum chromodynamicsarXiv:0908.3341.

[87] H.-T. Ding, F. Karsch, S. Mukherjee, Thermodynamics of strong-interaction matter from Lattice QCDarXiv:1504.05274.

[88] K. G. Wilson, Confinement of Quarks, Phys. Rev. D10 (1974) 2445-2459. doi:10.1103/ PhysRevD.10.2445.

[89] J. B. Kogut, L. Susskind, Hamiltonian Formulation of Wilson's Lattice Gauge Theories, Phys. Rev. D11 (1975) 395-408. doi:10.1103/PhysRevD.11.395.

[90] D. B. Kaplan, A Method for simulating chiral fermions on the lattice, Phys. Lett. B288 (1992) 342-347. arXiv:hep-lat/9206013, doi:10.1016/0370-2693(92)91112-M.

[91] H. Neuberger, Exactly massless quarks on the lattice, Phys. Lett. B417 (1998) 141-144. arXiv: hep-lat/9707022, doi:10.1016/S0370-2693(97)01368-3.

[92] P. Huovinen, P. Petreczky, C. Schmidt, Equation of state at finite net-baryon density using Taylor coefficients up to sixth order, Nucl. Phys. A931 (2014) 769-773. arXiv:1407.8532, doi:10.1016/j.nuclphysa.2014.08.069.

[93] M. Cristoforetti, F. Di Renzo, L. Scorzato, New approach to the sign problem in quantum field theories: High density QCD on a Lefschetz thimble, Phys. Rev. D86 (2012) 074506. arXiv:1205.3996, doi:10.1103/PhysRevD.86.074506.

[94] G. Aarts, L. Bongiovanni, E. Seiler, D. Sexty, Some remarks on Lefschetz thimbles and complex Langevin dynamics, JHEP 10 (2014) 159. arXiv:1407.2090, doi:10.1007/ JHEP10 (2014) 159.

[95] G. Aarts, E. Seiler, I.-O. Stamatescu, The Complex Langevin method: When can it be trusted?, Phys. Rev. D81 (2010) 054508. arXiv:0912.3360, doi:10.1103/PhysRevD.81. 054508.

[96] D. Sexty, Simulating full QCD at nonzero density using the complex Langevin equation, Phys. Lett. B729 (2014) 108-111. arXiv:1307.7748, doi:10.1016/j.physletb.2014.01. 019.

[97] T. Kloiber, C. Gattringer, Dual Methods for Lattice Field Theories at Finite Density, PoS LATTICE2013 (2014) 206. arXiv:1310.8535.

[98] H. B. Meyer, Transport Properties of the Quark-Gluon Plasma: A Lattice QCD Perspective, Eur. Phys. J. A47 (2011) 86. arXiv:1104.3708, doi:10.1140/epja/i2011-11086-3.

[99] F. Karsch, H. W. Wyld, Thermal Green's Functions and Transport Coefficients on the Lattice, Phys. Rev. D35 (1987) 2518. doi:10.1103/PhysRevD.35.2518.

[100] H. B. Meyer, A Calculation of the shear viscosity in SU(3) gluodynamics, Phys. Rev. D76 82 
(2007) 101701. arXiv:0704.1801, doi:10.1103/PhysRevD.76.101701.

[101] H. B. Meyer, A Calculation of the bulk viscosity in SU(3) gluodynamics, Phys. Rev. Lett. 100 (2008) 162001. arXiv:0710.3717, doi:10.1103/PhysRevLett.100.162001.

[102] S. Sakai, A. Nakamura, Lattice calculation of the QGP viscosities: Present results and next project, PoS LAT2007 (2007) 221, [AIP Conf. Proc.893,5(2007)]. arXiv:0710.3625, doi: $10.1063 / 1.2729742$.

[103] G. Aarts, C. Allton, J. Foley, S. Hands, S. Kim, Spectral functions at small energies and the electrical conductivity in hot, quenched lattice QCD, Phys. Rev. Lett. 99 (2007) 022002. arXiv:hep-lat/0703008, doi:10.1103/PhysRevLett.99.022002.

[104] G. Aarts, Transport and spectral functions in high-temperature QCD, PoS LAT2007 (2007) 001. arXiv:0710.0739.

[105] G. Aarts, C. Allton, J. Foley, S. Hands, S. Kim, Meson spectral functions at nonzero momentum in hot QCD, Nucl. Phys. A785 (2007) 202-205. arXiv:hep-lat/0607012, doi:10.1016/j.nuclphysa.2006.11.148.

[106] H. B. Meyer, Energy-momentum tensor correlators and spectral functions, JHEP 08 (2008) 031. arXiv:0806.3914, doi:10.1088/1126-6708/2008/08/031.

[107] P. Kovtun, D. T. Son, A. O. Starinets, Viscosity in strongly interacting quantum field theories from black hole physics, Phys. Rev. Lett. 94 (2005) 111601. arXiv:hep-th/ 0405231, doi:10.1103/PhysRevLett.94.111601.

[108] J. Adam, et al., Production of light nuclei and anti-nuclei in pp and $\mathrm{Pb}-\mathrm{Pb}$ collisions at LHC energiesarXiv: 1506.08951.

[109] A. Andronic, P. Braun-Munzinger, J. Stachel, Hadron production in central nucleusnucleus collisions at chemical freeze-out, Nucl. Phys. A772 (2006) 167-199. arXiv: nucl-th/0511071, doi:10.1016/j.nuclphysa.2006.03.012.

[110] F. Becattini, J. Manninen, M. Gazdzicki, Energy and system size dependence of chemical freeze-out in relativistic nuclear collisions, Phys. Rev. C73 (2006) 044905. arXiv:hep-ph/ 0511092, doi:10.1103/PhysRevC.73.044905.

[111] A. Andronic, P. Braun-Munzinger, J. Stachel, Thermal hadron production in relativistic nuclear collisions: The Hadron mass spectrum, the horn, and the QCD phase transition, Phys. Lett. B673 (2009) 142-145, [Erratum: Phys. Lett.B678,516(2009)]. arXiv:0812. 1186, doi:10.1016/j.physletb.2009.02.014,10.1016/j.physletb.2009.06.021.

[112] B. B. Abelev, et al., Multi-strange baryon production at mid-rapidity in $\mathrm{Pb}-\mathrm{Pb}$ collisions at $\sqrt{s_{N N}}=2.76 \mathrm{TeV}$, Phys. Lett. B728 (2014) 216-227, [Erratum: Phys. Lett.B734,409(2014)]. arXiv:1307.5543, doi:10.1016/j.physletb.2014.05.052,10. 1016/j.physletb. 2013.11.048.

[113] A. Andronic, An overview of the experimental study of quark-gluon matter in high-energy nucleus-nucleus collisions, Int. J. Mod. Phys. A29 (2014) 1430047. arXiv:1407.5003, doi:10.1142/S0217751X14300476.

[114] P. Braun-Munzinger, K. Redlich, J. Stachel, Particle production in heavy ion collisionsIn Quark Gluon Plasma 3, eds. R.C. Hwa and Xin-Nian Wang, World Scientific Publishing, Singapore, 2004.

[115] P. Braun-Munzinger, J. Stachel, C. Wetterich, Chemical freezeout and the QCD phase transition temperature, Phys. Lett. B596 (2004) 61-69. arXiv:nucl-th/0311005, doi: 10.1016/j.physletb.2004.05.081.

[116] R. Hagedorn, How We Got to QCD Matter From the Hadron Side by Trial and Error, Lect. Notes Phys. 221 (1985) 53-76.

[117] P. Braun-Munzinger, J. Stachel, Dynamics of ultrarelativistic nuclear collisions with heavy beams: An Experimental overview, Nucl. Phys. A638 (1998) 3-18. arXiv: nucl-ex/9803015, doi:10.1016/S0375-9474(98)00342-X.

[118] R. Stock, The Parton to hadron phase transition observed in $\mathrm{Pb}+\mathrm{Pb}$ collisions at 158 GeV per nucleon, Phys. Lett. B456 (1999) 277-282. arXiv:hep-ph/9905247, doi:10. 
1016/S0370-2693(99) 00482-7.

[119] P. Braun-Munzinger, J. Stachel, Particle ratios, equilibration, and the QCD phase boundary, J. Phys. G28 (2002) 1971-1976. arXiv:nucl-th/0112051, doi:10.1088/0954-3899/ $28 / 7 / 355$.

[120] A. Andronic, et al., Hadron Production in Ultra-relativistic Nuclear Collisions: Quarkyonic Matter and a Triple Point in the Phase Diagram of QCD, Nucl. Phys. A837 (2010) 65-86. arXiv:0911.4806, doi:10.1016/j.nuclphysa.2010.02.005.

[121] S. Floerchinger, C. Wetterich, Chemical freeze-out in heavy ion collisions at large baryon densities, Nucl. Phys. A890-891 (2012) 11-24. arXiv:1202.1671, doi:10.1016/ j.nuclphysa.2012.07.009.

[122] J. Stachel, A. Andronic, P. Braun-Munzinger, K. Redlich, Confronting LHC data with the statistical hadronization model, J. Phys. Conf. Ser. 509 (2014) 012019. arXiv:1311.4662, doi:10.1088/1742-6596/509/1/012019.

[123] B. B. Abelev, et al., Performance of the ALICE Experiment at the CERN LHC, Int. J. Mod. Phys. A29 (2014) 1430044. arXiv:1402.4476, doi:10.1142/S0217751X14300440.

[124] M. Floris, Hadron yields and the phase diagram of strongly interacting matter, Nucl. Phys. A931 (2014) 103-112. arXiv:1408.6403, doi:10.1016/j.nuclphysa.2014.09.002.

[125] P. Braun-Munzinger, B. Doenigus, N. Loeher, Snow balls in hell, Cern Courier 55 (2015) $14-15$.

[126] A. Andronic, P. Braun-Munzinger, K. Redlich, J. Stachel, The thermal model on the verge of the ultimate test: particle production in $\mathrm{Pb}-\mathrm{Pb}$ collisions at the LHC, J. Phys. G38 (2011) 124081. arXiv:1106.6321, doi:10.1088/0954-3899/38/12/124081.

[127] B. Abelev, et al., Pion, Kaon, and Proton Production in Central Pb-Pb Collisions at $\sqrt{s_{N N}}=2.76 \mathrm{TeV}$, Phys. Rev. Lett. 109 (2012) 252301. arXiv:1208.1974, doi:10.1103/ PhysRevLett.109.252301.

[128] G. F. Chapline, A. K. Kerman, On the Possibility of Making Quark Matter in Nuclear Collisions, MIT-CTP-695 .

[129] A. Tawfik, On the conditions driving the chemical freeze-out, Europhys. Lett. 75 (2006) 420. arXiv:hep-ph/0410392, doi:10.1209/epl/i2006-10119-4.

[130] J. Cleymans, H. Oeschler, K. Redlich, S. Wheaton, Comparison of chemical freeze-out criteria in heavy-ion collisions, Phys. Rev. C73 (2006) 034905. arXiv:hep-ph/0511094, doi:10.1103/PhysRevC.73.034905.

[131] J. Cleymans, H. Oeschler, K. Redlich, Influence of impact parameter on thermal description of relativistic heavy ion collisions at (1-2) A-GeV, Phys. Rev. C59 (1999) 1663. arXiv:nucl-th/9809027, doi:10.1103/PhysRevC.59.1663.

[132] J. Manninen, F. Becattini, Chemical freeze-out in ultra-relativistic heavy ion collisions at $s_{N N}^{1 / 2}=130$ and 200-GeV, Phys. Rev. C78 (2008) 054901. arXiv:0806.4100, doi: 10.1103/PhysRevC.78.054901.

[133] B. I. Abelev, et al., Identified particle production, azimuthal anisotropy, and interferometry measurements in $\mathrm{Au}+\mathrm{Au}$ collisions at $s_{N N}^{1 / 2}=9.2-\mathrm{GeV}$, Phys. Rev. C81 (2010) 024911. arXiv:0909.4131, doi:10.1103/PhysRevC.81.024911.

[134] M. M. Aggarwal, et al., Scaling properties at freeze-out in relativistic heavy ion collisions, Phys. Rev. C83 (2011) 034910. arXiv:1008.3133, doi:10.1103/PhysRevC.83.034910.

[135] M. Gazdzicki, M. I. Gorenstein, On the early stage of nucleus-nucleus collisions, Acta Phys. Polon. B30 (1999) 2705. arXiv:hep-ph/9803462.

[136] C. Alt, et al., Pion and kaon production in central $\mathrm{Pb}+\mathrm{Pb}$ collisions at 20-A and 30A-GeV: Evidence for the onset of deconfinement, Phys. Rev. C77 (2008) 024903. arXiv: 0710.0118, doi:10.1103/PhysRevC.77.024903.

[137] A. Andronic, P. Braun-Munzinger, J. Stachel, H. Stocker, Production of light nuclei, hypernuclei and their antiparticles in relativistic nuclear collisions, Phys. Lett. B697 (2011) 
203-207. arXiv:1010.2995, doi:10.1016/j.physletb.2011.01.053.

[138] J. Letessier, J. Rafelski, Hadron production and phase changes in relativistic heavy ion collisions, Eur. Phys. J. A35 (2008) 221-242. arXiv:nucl-th/0504028, doi:10.1140/ epja/i2007-10546-7.

[139] M. Petran, J. Rafelski, Universal hadronization condition in heavy ion collisions at $\sqrt{s_{\mathrm{NN}}}=$ $62 \mathrm{GeV}$ and at $\sqrt{s_{\mathrm{NN}}}=2.76 \mathrm{TeV}$, Phys. Rev. C88 (2) (2013) 021901. arXiv:1303.0913, doi:10.1103/PhysRevC.88.021901.

[140] A. Dumitru, L. Portugal, D. Zschiesche, Inhomogeneous freeze-out in relativistic heavyion collisions, Phys. Rev. C73 (2006) 024902. arXiv:nucl-th/0511084, doi:10.1103/ PhysRevC.73.024902.

[141] A. Andronic, P. Braun-Munzinger, J. Stachel, M. Winn, Interacting hadron resonance gas meets lattice QCD, Phys. Lett. B718 (2012) 80-85. arXiv:1201.0693, doi:10.1016/j. physletb.2012.10.001.

[142] D. H. Rischke, M. I. Gorenstein, H. Stoecker, W. Greiner, Excluded volume effect for the nuclear matter equation of state, Z. Phys. C51 (1991) 485-490. doi:10.1007/BF01548574.

[143] F. Becattini, E. Grossi, M. Bleicher, J. Steinheimer, R. Stock, Centrality dependence of hadronization and chemical freeze-out conditions in heavy ion collisions at $\sqrt{s}_{N N}=2.76$ TeV, Phys. Rev. C90 (5) (2014) 054907. arXiv:1405.0710, doi:10.1103/PhysRevC.90. 054907.

[144] A. Bazavov, et al., Additional Strange Hadrons from QCD Thermodynamics and Strangeness Freezeout in Heavy Ion Collisions, Phys. Rev. Lett. 113 (7) (2014) 072001. arXiv:1404.6511, doi:10.1103/PhysRevLett.113.072001.

[145] A. Bazavov, et al., The melting and abundance of open charm hadrons, Phys. Lett. B737 (2014) 210-215. arXiv:1404.4043, doi:10.1016/j.physletb.2014.08.034.

[146] A. Andronic, F. Beutler, P. Braun-Munzinger, K. Redlich, J. Stachel, Thermal description of hadron production in e+e- collisions revisited, Phys. Lett. B675 (2009) 312-318. arXiv : 0804.4132, doi:10.1016/j.physletb.2009.04.024.

[147] F. Becattini, P. Castorina, A. Milov, H. Satz, A Comparative analysis of statistical hadron production, Eur. Phys. J. C66 (2010) 377-386. arXiv:0911.3026, doi:10.1140/epjc/ s10052-010-1265-y.

[148] U. W. Heinz, Early collective expansion: Relativistic hydrodynamics and the transport properties of QCD matter, in: R. Stock (Ed.), Relativistic Heavy ion Collisions, Vol. I/23 of Landolt-Boernstein New Series I/23, Springer, 2010, pp. 5-1. arXiv:0901.4355.

[149] D. A. Teaney, Viscous Hydrodynamics and the Quark Gluon Plasma, in: R. C. Hwa, X. N. Wang (Eds.), Quark Gluon Plasma 4, World Scientific, 2010. arXiv:0905.2433.

[150] P. Romatschke, New Developments in Relativistic Viscous Hydrodynamics, Int. J. Mod. Phys. E19 (2010) 1-53. arXiv:0902.3663, doi:10.1142/S0218301310014613.

[151] T. Schäfer, D. Teaney, Nearly Perfect Fluidity: From Cold Atomic Gases to Hot Quark Gluon Plasmas, Rept.Prog.Phys. 72 (2009) 126001. arXiv:0904.3107, doi:10.1088/ 0034-4885/72/12/126001.

[152] T. Schäfer, Fluid Dynamics and Viscosity in Strongly Correlated Fluids, Ann. Rev. Nucl. Part. Sci. 64 (2014) 125-148. arXiv:1403.0653, doi:10.1146/ annurev-nucl-102313-025439.

[153] K. Dusling, T. Schäfer, Bulk viscosity, particle spectra and flow in heavy-ion collisions, Phys. Rev. C85 (2012) 044909. arXiv:1109.5181, doi:10.1103/PhysRevC.85.044909.

[154] D. H. Rischke, Fluid dynamics for relativistic nuclear collisions[Lect. Notes Phys.516,21(1999)]. arXiv:nucl-th/9809044, doi:10.1007/BFb0107310.

[155] A. Muronga, Second order dissipative fluid dynamics for ultrarelativistic nuclear collisions, Phys. Rev. Lett. 88 (2002) 062302, [Erratum: Phys. Rev. Lett.89,159901(2002)]. arXiv: nucl-th/0104064, doi:10.1103/PhysRevLett.88.062302.

[156] L. D. Landau, E. M. Lifshitz, Hydrodynamics, Course of Theoretical Physics, Vol.VI, 
Pergamon Press, New York, 1980.

[157] W. A. Hiscock, L. Lindblom, Generic instabilities in first-order dissipative relativistic fluid theories, Phys. Rev. D31 (1985) 725-733. doi:10.1103/PhysRevD.31.725.

[158] R. Baier, P. Romatschke, D. T. Son, A. O. Starinets, M. A. Stephanov, Relativistic viscous hydrodynamics, conformal invariance, and holography, JHEP 04 (2008) 100. arXiv: 0712.2451, doi:10.1088/1126-6708/2008/04/100.

[159] W. Israel, J. M. Stewart, Transient relativistic thermodynamics and kinetic theory, Annals Phys. 118 (1979) 341-372. doi:10.1016/0003-4916(79)90130-1.

[160] G. S. Denicol, H. Niemi, E. Molnar, D. H. Rischke, Derivation of transient relativistic fluid dynamics from the Boltzmann equation, Phys. Rev. D85 (2012) 114047, [Erratum: Phys. Rev.D91,no.3,039902(2015)]. arXiv:1202.4551, doi:10.1103/PhysRevD.85.114047, 10. 1103/PhysRevD.91.039902.

[161] R. Kubo, Statistical-mechanical theory of irreversible processes: General theory and simple applications to magnetic and conduction problems, Journal of the Physical Society of Japan 12 (6) (1957) 570-586. doi:10.1143/JPSJ.12.570.

[162] D. T. Son, A. O. Starinets, Viscosity, Black Holes, and Quantum Field Theory, Ann. Rev. Nucl. Part. Sci. 57 (2007) 95-118. arXiv:0704.0240, doi:10.1146/annurev.nucl. 57.090506 .123120$.

[163] M. A. York, G. D. Moore, Second order hydrodynamic coefficients from kinetic theory, Phys. Rev. D79 (2009) 054011. arXiv:0811.0729, doi:10.1103/PhysRevD.79.054011.

[164] G. Baym, H. Monien, C. J. Pethick, D. G. Ravenhall, Transverse Interactions and Transport in Relativistic Quark - Gluon and Electromagnetic Plasmas, Phys. Rev. Lett. 64 (1990) 1867-1870. doi:10.1103/PhysRevLett.64.1867.

[165] P. B. Arnold, G. D. Moore, L. G. Yaffe, Transport coefficients in high temperature gauge theories. 1. Leading log results, JHEP 11 (2000) 001. arXiv:hep-ph/0010177, doi:10. 1088/1126-6708/2000/11/001.

[166] P. B. Arnold, G. D. Moore, L. G. Yaffe, Transport coefficients in high temperature gauge theories. 2. Beyond leading log, JHEP 05 (2003) 051. arXiv:hep-ph/0302165, doi:10. $1088 / 1126-6708 / 2003 / 05 / 051$.

[167] S. S. Adler, et al., Elliptic flow of identified hadrons in au + au collisions at $\sqrt{s_{N N}}=$ 200-gev, Phys. Rev. Lett. 91 (2003) 182301. arXiv:nucl-ex/0305013.

[168] J. Adams, et al., Azimuthal anisotropy in Au+Au collisions at $\sqrt{s_{N N}}=200-\mathrm{GeV}$, Phys. Rev. C72 (2005) 014904. arXiv:nucl-ex/0409033, doi:10.1103/PhysRevC.72.014904.

[169] K. Aamodt, et al., Elliptic flow of charged particles in Pb-Pb collisions at 2.76 TeV, Phys. Rev. Lett. 105 (2010) 252302. arXiv:1011.3914, doi:10.1103/PhysRevLett.105.252302.

[170] P. Danielewicz, M. Gyulassy, Dissipative Phenomena in Quark Gluon Plasmas, Phys. Rev. D31 (1985) 53-62. doi:10.1103/PhysRevD.31.53.

[171] L. D. Landau, E. M. Lifshitz, Physical Kinetics, Course of Theoretical Physics, Vol.X, Pergamon Press, New York, 1981.

[172] J. Kapusta, B. Muller, M. Stephanov, Relativistic Theory of Hydrodynamic Fluctuations with Applications to Heavy Ion Collisions, Phys.Rev. C85 (2012) 054906. arXiv:1112. 6405, doi: 10.1103/PhysRevC.85.054906.

[173] C. Young, Numerical integration of thermal noise in relativistic hydrodynamics, Phys. Rev. C89 (2) (2014) 024913. arXiv:1306.0472, doi:10.1103/PhysRevC.89.024913.

[174] K. Murase, T. Hirano, Relativistic fluctuating hydrodynamics with memory functions and colored noisesarXiv: 1304.3243.

[175] P. Kovtun, G. D. Moore, P. Romatschke, The stickiness of sound: An absolute lower limit on viscosity and the breakdown of second order relativistic hydrodynamics, Phys. Rev. D84 (2011) 025006. arXiv:1104.1586, doi:10.1103/PhysRevD.84.025006.

[176] S. A. Voloshin, A. M. Poskanzer, R. Snellings, Collective phenomena in non-central nuclear collisions, in: Hadronic Fluctuations and Correlations, Landolt-Boernstein New Series 
I/23, Springer, 2010. arXiv:0809.2949.

[177] U. Heinz, R. Snellings, Collective flow and viscosity in relativistic heavy-ion collisions, Ann.Rev.Nucl.Part.Sci. 63 (2013) 123-151. arXiv:1301.2826, doi:10.1146/ annurev-nucl-102212-170540.

[178] E. Schnedermann, J. Sollfrank, U. W. Heinz, Thermal phenomenology of hadrons from 200-A/GeV S+S collisions, Phys. Rev. C48 (1993) 2462-2475. arXiv:nucl-th/9307020, doi: 10.1103/PhysRevC.48.2462.

[179] J. Adams, et al., Identified particle distributions in pp and $\mathrm{Au}+\mathrm{Au}$ collisions at $\sqrt{s_{N N}}$ $=200 \mathrm{GeV}$, Phys. Rev. Lett. 92 (2004) 112301. arXiv:nucl-ex/0310004, doi:10.1103/ PhysRevLett.92.112301.

[180] J.-Y. Ollitrault, Anisotropy as a signature of transverse collective flow, Phys. Rev. D46 (1992) 229-245. doi:10.1103/PhysRevD.46.229.

[181] B. Alver, G. Roland, Collision geometry fluctuations and triangular flow in heavy-ion collisions, Phys. Rev. C81 (2010) 054905, [Erratum: Phys. Rev.C82,039903(2010)]. arXiv : 1003.0194, doi:10.1103/PhysRevC.82.039903, 10.1103/PhysRevC.81.054905.

[182] B. Alver, et al., Importance of correlations and fluctuations on the initial source eccentricity in high-energy nucleus-nucleus collisions, Phys. Rev. C77 (2008) 014906. arXiv: 0711.3724, doi:10.1103/PhysRevC.77.014906.

[183] J. D. Bjorken, Highly Relativistic Nucleus-Nucleus Collisions: The Central Rapidity Region, Phys. Rev. D27 (1983) 140-151. doi:10.1103/PhysRevD.27.140.

[184] S. S. Gubser, A. Yarom, Conformal hydrodynamics in Minkowski and de Sitter spacetimes, Nucl. Phys. B846 (2011) 469-511. arXiv:1012.1314, doi:10.1016/j.nuclphysb. 2011. 01.012.

[185] B. Schenke, P. Tribedy, R. Venugopalan, Fluctuating Glasma initial conditions and flow in heavy ion collisions, Phys. Rev. Lett. 108 (2012) 252301. arXiv:1202.6646, doi: 10.1103/PhysRevLett.108.252301.

[186] D. Kharzeev, M. Nardi, Hadron production in nuclear collisions at RHIC and high density QCD, Phys. Lett. B507 (2001) 121-128. arXiv:nucl-th/0012025, doi:10.1016/ S0370-2693(01) 00457-9.

[187] F. Cooper, G. Frye, Comment on the Single Particle Distribution in the Hydrodynamic and Statistical Thermodynamic Models of Multiparticle Production, Phys. Rev. D10 (1974) 186. doi:10.1103/PhysRevD.10.186.

[188] P. L. Bhatnagar, E. P. Gross, M. Krook, A Model for Collision Processes in Gases. 1. Small Amplitude Processes in Charged and Neutral One-Component Systems, Phys. Rev. 94 (1954) 511-525. doi:10.1103/PhysRev.94.511.

[189] D. Teaney, The Effects of viscosity on spectra, elliptic flow, and HBT radii, Phys. Rev. C68 (2003) 034913. arXiv:nucl-th/0301099, doi:10.1103/PhysRevC.68.034913.

[190] S. Z. Belenkij, L. D. Landau, Hydrodynamic theory of multiple production of particles, Nuovo Cim. Suppl. 3S10 (1956) 15, [Usp. Fiz. Nauk56,309(1955)]. doi:10.1007/ BF02745507.

[191] G. Aad, et al., Measurement of the azimuthal anisotropy for charged particle production in $\sqrt{s_{N N}}=2.76 \mathrm{TeV}$ lead-lead collisions with the ATLAS detector, Phys. Rev. C86 (2012) 014907. arXiv:1203.3087, doi:10.1103/PhysRevC.86.014907.

[192] C. Gale, S. Jeon, B. Schenke, P. Tribedy, R. Venugopalan, Event-by-event anisotropic flow in heavy-ion collisions from combined Yang-Mills and viscous fluid dynamics, Phys. Rev. Lett. 110 (1) (2013) 012302. arXiv:1209.6330, doi:10.1103/PhysRevLett.110.012302.

[193] C. Gale, S. Jeon, B. Schenke, Hydrodynamic Modeling of Heavy-Ion Collisions, Int.J.Mod.Phys. A28 (2013) 1340011. arXiv:1301.5893, doi:10.1142/ S0217751X13400113.

[194] M. L. Miller, K. Reygers, S. J. Sanders, P. Steinberg, Glauber modeling in high energy nuclear collisions, Ann. Rev. Nucl. Part. Sci. 57 (2007) 205-243. arXiv:nucl-ex/0701025, 
doi:10.1146/annurev.nucl.57.090506.123020.

[195] M. Martinez, M. Strickland, Dissipative Dynamics of Highly Anisotropic Systems, Nucl. Phys. A848 (2010) 183-197. arXiv:1007.0889, doi:10.1016/j.nuclphysa.2010.08.011.

[196] H. Petersen, J. Steinheimer, G. Burau, M. Bleicher, H. Stocker, A Fully Integrated Transport Approach to Heavy Ion Reactions with an Intermediate Hydrodynamic Stage, Phys. Rev. C78 (2008) 044901. arXiv:0806.1695, doi:10.1103/PhysRevC.78.044901.

[197] W. van der Schee, P. Romatschke, S. Pratt, Fully Dynamical Simulation of Central Nuclear Collisions, Phys.Rev.Lett. 111 (22) (2013) 222302. arXiv:1307.2539, doi: 10.1103/PhysRevLett.111.222302.

[198] P. Huovinen, P. Petreczky, QCD Equation of State and Hadron Resonance Gas, Nucl. Phys. A837 (2010) 26-53. arXiv:0912.2541, doi:10.1016/j.nuclphysa.2010.02.015.

[199] S. A. Bass, A. Dumitru, Dynamics of hot bulk QCD matter: From the quark gluon plasma to hadronic freezeout, Phys. Rev. C61 (2000) 064909. arXiv:nucl-th/0001033, doi:10.1103/PhysRevC.61.064909.

[200] T. Hirano, U. W. Heinz, D. Kharzeev, R. Lacey, Y. Nara, Hadronic dissipative effects on elliptic flow in ultrarelativistic heavy-ion collisions, Phys. Lett. B636 (2006) 299-304. arXiv:nucl-th/0511046, doi:10.1016/j.physletb.2006.03.060.

[201] D. Teaney, J. Lauret, E. V. Shuryak, A Hydrodynamic description of heavy ion collisions at the SPS and RHICarXiv:nucl-th/0110037.

[202] T. Hirano, M. Gyulassy, Perfect fluidity of the quark gluon plasma core as seen through its dissipative hadronic corona, Nucl.Phys. A769 (2006) 71-94. arXiv:nucl-th/0506049, doi:10.1016/j.nuclphysa.2006.02.005.

[203] A. Adare, et al., Heavy Quark Production in $p+p$ and Energy Loss and Flow of Heavy Quarks in $\mathrm{Au}+\mathrm{Au}$ Collisions at $\sqrt{s_{N N}}=200 \mathrm{GeV}$, Phys. Rev. C84 (2011) 044905. arXiv: 1005.1627, doi:10.1103/PhysRevC.84.044905.

[204] G. D. Moore, D. Teaney, How much do heavy quarks thermalize in a heavy ion collision?, Phys. Rev. C71 (2005) 064904. arXiv:hep-ph/0412346, doi:10.1103/PhysRevC. 71.064904.

[205] H. Song, U. W. Heinz, Causal viscous hydrodynamics in $2+1$ dimensions for relativistic heavy-ion collisions, Phys. Rev. C77 (2008) 064901. arXiv:0712.3715, doi:10.1103/ PhysRevC.77.064901.

[206] H. Song, S. A. Bass, U. Heinz, Elliptic flow in $200 \mathrm{~A} \mathrm{GeV} \mathrm{Au+Au} \mathrm{collisions} \mathrm{and} \mathrm{2.76} \mathrm{A} \mathrm{TeV}$ $\mathrm{Pb}+\mathrm{Pb}$ collisions: insights from viscous hydrodynamics + hadron cascade hybrid model, Phys. Rev. C83 (2011) 054912, [Erratum: Phys. Rev.C87,no.1,019902(2013)]. arXiv: 1103.2380, doi:10.1103/PhysRevC.83.054912,10.1103/PhysRevC.87.019902.

[207] M. Luzum, J.-Y. Ollitrault, Extracting the shear viscosity of the quark-gluon plasma from flow in ultra-central heavy-ion collisions, Nucl. Phys. A904-905 (2013) 377c-380c. arXiv:1210.6010, doi:10.1016/j.nuclphysa.2013.02.028.

[208] H. Song, U. W. Heinz, Extracting the QGP viscosity from RHIC data - A Status report from viscous hydrodynamics, J. Phys. G36 (2009) 064033. arXiv:0812.4274, doi:10. 1088/0954-3899/36/6/064033.

[209] C. Shen, U. Heinz, Collision Energy Dependence of Viscous Hydrodynamic Flow in Relativistic Heavy-Ion Collisions, Phys. Rev. C85 (2012) 054902, [Erratum: Phys. Rev.C86,049903(2012)]. arXiv:1202.6620, doi:10.1103/PhysRevC.86.049903,10.1103/ PhysRevC.85.054902.

[210] S. Chatrchyan, et al., Multiplicity and transverse momentum dependence of two- and four-particle correlations in pPb and PbPb collisions, Phys. Lett. B724 (2013) 213-240. arXiv: 1305.0609, doi:10.1016/j.physletb.2013.06.028.

[211] G. Aad, et al., Measurement with the ATLAS detector of multi-particle azimuthal cor-

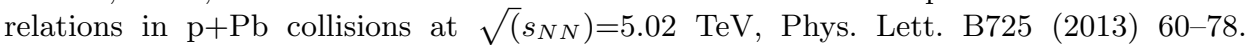
arXiv:1303.2084, doi:10.1016/j.physletb.2013.06.057. 
[212] B. B. Abelev, et al., Long-range angular correlations of $\pi, \mathrm{K}$ and $\mathrm{p}$ in $\mathrm{p}-\mathrm{Pb}$ collisions at $\sqrt{s_{\mathrm{NN}}}=5.02 \mathrm{TeV}$, Phys. Lett. B726 (2013) 164-177. arXiv:1307.3237, doi:10.1016/j . physletb.2013.08.024.

[213] K. Dusling, R. Venugopalan, Comparison of the color glass condensate to dihadron correlations in proton-proton and proton-nucleus collisions, Phys. Rev. D87 (9) (2013) 094034 arXiv:1302.7018, doi:10.1103/PhysRevD.87.094034.

[214] L. McLerran, M. Praszalowicz, B. Schenke, Transverse Momentum of Protons, Pions and Kaons in High Multiplicity pp and pA Collisions: Evidence for the Color Glass Condensate?, Nucl. Phys. A916 (2013) 210-218. arXiv:1306.2350, doi:10.1016/j.nuclphysa. 2013.08 .008$.

[215] P. Romatschke, Collective flow without hydrodynamics: simulation results for relativistic ion collisionsarXiv: 1504.02529.

[216] G. Basarar, D. Teaney, Scaling relation between pA and AA collisions, Phys. Rev. C90 (5) (2014) 054903. arXiv:1312.6770, doi:10.1103/PhysRevC.90.054903.

[217] H. Heiselberg, A.-M. Levy, Elliptic flow and HBT in noncentral nuclear collisions, Phys. Rev. C59 (1999) 2716-2727. arXiv:nucl-th/9812034, doi:10.1103/PhysRevC.59.2716.

[218] R. S. Bhalerao, J.-P. Blaizot, N. Borghini, J.-Y. Ollitrault, Elliptic flow and incomplete equilibration at RHIC, Phys. Lett. B627 (2005) 49-54. arXiv:nucl-th/0508009, doi: 10.1016/j.physletb.2005.08.131.

[219] S. A. Voloshin, A. M. Poskanzer, The Physics of the centrality dependence of elliptic flow, Phys. Lett. B474 (2000) 27-32. arXiv:nucl-th/9906075, doi:10.1016/S0370-2693(00) 00017-4.

[220] C. Alt, et al., Directed and elliptic flow of charged pions and protons in $\mathrm{Pb}+\mathrm{Pb}$ collisions at 40-A-GeV and 158-A-GeV, Phys. Rev. C68 (2003) 034903. arXiv:nucl-ex/0303001, doi:10.1103/PhysRevC.68.034903.

[221] V. Khachatryan, et al., Observation of Long-Range Near-Side Angular Correlations in Proton-Proton Collisions at the LHC, JHEP 09 (2010) 091. arXiv:1009.4122, doi:10. 1007/JHEP09 (2010) 091.

[222] The ATLAS Collaboration, Measurement of twoparticle correlations in $\sqrt{s}=13 \mathrm{TeV}$ protonproton collisions at the LHC with the ATLAS detector, ATLAS-CONF-2015-027 (2015).

[223] A. Adare, et al., Observation of direct-photon collective flow in $\sqrt{s_{N N}}=200 \mathrm{GeV}$ $\mathrm{Au}+\mathrm{Au}$ collisions, Phys. Rev. Lett. 109 (2012) 122302. arXiv:1105.4126, doi:10.1103/ PhysRevLett.109.122302.

[224] D. Lohner, Measurement of Direct-Photon Elliptic Flow in Pb-Pb Collisions at $\sqrt{s_{N N}}=$ 2.76 TeV, J. Phys. Conf. Ser. 446 (2013) 012028. arXiv:1212.3995, doi:10.1088/ 1742-6596/446/1/012028.

[225] R. Chatterjee, E. S. Frodermann, U. W. Heinz, D. K. Srivastava, Elliptic flow of thermal photons in relativistic nuclear collisions, Phys. Rev. Lett. 96 (2006) 202302. arXiv:nucl-th/0511079, doi:10.1103/PhysRevLett.96.202302.

[226] J.-F. Paquet, C. Shen, G. S. Denicol, M. Luzum, B. Schenke, S. Jeon, C. Gale, The production of photons in relativistic heavy-ion collisionsarXiv:1509.06738.

[227] S. Chatrchyan, et al., Measurement of the elliptic anisotropy of charged particles produced in $\mathrm{PbPb}$ collisions at $\sqrt{s_{N N}}=2.76 \mathrm{TeV}$, Phys. Rev. C87 (1) (2013) 014902. arXiv: 1204 . 1409, doi:10.1103/PhysRevC.87.014902.

[228] L. Adamczyk, et al., Inclusive charged hadron elliptic flow in $\mathrm{Au}+\mathrm{Au}$ collisions at $\sqrt{s_{N N}}=7.7-39 \mathrm{GeV}$, Phys. Rev. C86 (2012) 054908. arXiv:1206.5528, doi:10.1103/ PhysRevC.86.054908.

[229] D. E. Kharzeev, L. D. McLerran, H. J. Warringa, The Effects of topological charge change in heavy ion collisions: 'Event by event P and CP violation', Nucl.Phys. A803 (2008) 227 253. arXiv:0711.0950, doi:10.1016/j.nuclphysa.2008.02.298. 
[230] D. E. Kharzeev, D. T. Son, Testing the chiral magnetic and chiral vortical effects in heavy ion collisions, Phys. Rev. Lett. 106 (2011) 062301. arXiv:1010.0038, doi:10. 1103/PhysRevLett.106.062301.

[231] L. Adamczyk, et al., Observation of charge asymmetry dependence of pion elliptic flow and the possible chiral magnetic wave in heavy-ion collisions, Phys. Rev. Lett. 114 (25) (2015) 252302. arXiv:1504.02175, doi:10.1103/PhysRevLett.114.252302.

[232] D. E. Kharzeev, H.-U. Yee, Chiral Magnetic Wave, Phys. Rev. D83 (2011) 085007. arXiv: 1012.6026, doi:10.1103/PhysRevD.83.085007.

[233] G. F. Smoot, Nobel Lecture: Cosmic microwave background radiation anisotropies: Their discovery and utilization, Rev. Mod. Phys. 79 (2007) 1349-1379. doi:10.1103/ RevModPhys.79.1349.

[234] D. N. Spergel, et al., Wilkinson Microwave Anisotropy Probe (WMAP) three year results: Implications for cosmology, Astrophys. J. Suppl. 170 (2007) 377. arXiv:astro-ph/ 0603449, doi:10.1086/513700.

[235] P. Ade, et al., Planck 2013 results. XVI. Cosmological parameters, Astron.Astrophys.arXiv: 1303.5076, doi:10.1051/0004-6361/201321591.

[236] A. Bialas, V. Koch, Event by event fluctuations and inclusive distribution, Phys.Lett. B456 (1999) 1-4. arXiv:nucl-th/9902063, doi:10.1016/S0370-2693(99)00479-7.

[237] V. Koch, Hadronic Fluctuations and Correlations, in: R. Stock (Ed.), Relativistic Heavy ion Collisions, Vol. I/23 of Landolt-Boernstein New Series I/23, Springer, 2010, pp. 8-1. arXiv:0810.2520.

[238] S. Borsanyi, G. Endrodi, Z. Fodor, S. Katz, S. Krieg, et al., QCD equation of state at nonzero chemical potential: continuum results with physical quark masses at order $m u^{2}$, JHEP 1208 (2012) 053. arXiv:1204.6710, doi:10.1007/JHEP08(2012) 053.

[239] S. Jeon, V. Koch, Charged particle ratio fluctuation as a signal for QGP, Phys.Rev.Lett. 85 (2000) 2076-2079. arXiv:hep-ph/0003168, doi:10.1103/PhysRevLett.85. 2076.

[240] M. Asakawa, U. W. Heinz, B. Muller, Fluctuation probes of quark deconfinement, Phys. Rev. Lett. 85 (2000) 2072-2075. arXiv:hep-ph/0003169.

[241] S. Jeon, V. Koch, Event-by-event fluctuations, in: R. Hwa, X. Wang (Eds.), Quark Gluon Plasma 3, World Scientific, Singapore, 2004, pp. 430-490. arXiv:hep-ph/0304012.

[242] L. Saminadayar, D. C. Glattli, Y. Jin, B. Etienne, Observation of the $e / 3$ fractionally charged laughlin quasiparticle, Phys. Rev. Lett. 79 (1997) 2526-2529. doi:10.1103/ PhysRevLett.79.2526.

URL http://link.aps.org/doi/10.1103/PhysRevLett.79.2526

[243] X. Jehl, M. Sanquer, R. Calemczuk, D. Mailly, Detection of doubled shot noise in short normal-metal superconductor junctions, Nature 405 (2000) 50.

[244] S. Borsanyi, Z. Fodor, S. D. Katz, S. Krieg, C. Ratti, et al., Fluctuations of conserved charges at finite temperature from lattice QCD, JHEP 1201 (2012) 138. arXiv:1112.4416, doi:10.1007/JHEP01 (2012) 138.

[245] V. Koch, A. Majumder, J. Randrup, Baryon-strangeness correlations: A Diagnostic of strongly interacting matter, Phys.Rev.Lett. 95 (2005) 182301. arXiv:nucl-th/0505052, doi:10.1103/PhysRevLett.95.182301.

[246] A. Bazavov, et al., Fluctuations and Correlations of net baryon number, electric charge and strangeness: A comparison of lattice QCD results with the hadron resonance gas model, Phys.Rev. D86 (2012) 034509. arXiv:1203.0784, doi:10.1103/PhysRevD.86. 034509.

[247] K. A. Olive, et al., Review of Particle Physics, Chin. Phys. C38 (2014) 090001. doi: 10.1088/1674-1137/38/9/090001.

[248] A. Bazavov, H. T. Ding, P. Hegde, O. Kaczmarek, F. Karsch, et al., Strangeness at high temperatures: from hadrons to quarks, Phys. Rev. Lett. 111 (2013) 082301. arXiv: 1304.7220, doi:10.1103/PhysRevLett.111.082301. 
[249] E. V. Shuryak, M. A. Stephanov, When can long range charge fluctuations serve as a qgp signal?, Phys. Rev. C63 (2001) 064903. arXiv:hep-ph/0010100.

[250] M. Kitazawa, M. Asakawa, H. Ono, Non-equilibrium time evolution of higher order cumulants of conserved charges and event-by-event analysis, Phys.Lett. B728 (2014) 386-392. arXiv:1307.2978, doi:10.1016/j.physletb.2013.12.008.

[251] A. Bzdak, V. Koch, V. Skokov, Baryon number conservation and the cumulants of the net proton distribution, Phys.Rev. C87 (2013) 014901. arXiv:1203.4529, doi:10.1103/ PhysRevC.87.014901.

[252] V. Koch, M. Bleicher, S. Jeon, Event-by-event fluctuations and the QGP, Nucl.Phys. A698 (2002) 261-268. arXiv:nucl-th/0103084, doi:10.1016/S0375-9474(01)01372-0.

[253] M. A. Aziz, S. Gavin, Causal diffusion and the survival of charge fluctuations in nuclear collisions, Phys. Rev. C70 (2004) 034905. arXiv:nucl-th/0404058, doi:10.1103/ PhysRevC.70.034905.

[254] M. Kitazawa, M. Asakawa, Relation between baryon number fluctuations and experimentally observed proton number fluctuations in relativistic heavy ion collisions, Phys.Rev. C86 (2012) 024904. arXiv:1205.3292, doi:10.1103/PhysRevC.86.024904,10.1103/ PhysRevC.86.069902.

[255] X. Luo, Energy Dependence of Moments of Net-Proton and Net-Charge Multiplicity Distributions at STAR, PoS CPOD2014 (2014) 019. arXiv:1503.02558.

[256] A. Bzdak, V. Koch, Acceptance corrections to net baryon and net charge cumulants, Phys.Rev. C86 (2012) 044904. arXiv:1206.4286, doi:10.1103/PhysRevC.86.044904.

[257] A. Bzdak, V. Koch, Local Efficiency Corrections to Higher Order Cumulants, Phys. Rev. C91 (2) (2015) 027901. arXiv:1312.4574, doi:10.1103/PhysRevC.91.027901.

[258] X. Luo, Unified Description of Efficiency Correction and Error Estimation for Moments of Conserved Quantities in Heavy-Ion Collisions, Phys. Rev. C91 (3) (2015) 034907. arXiv: 1410.3914, doi:10.1103/PhysRevC.91.034907.

[259] V. Skokov, B. Friman, K. Redlich, Volume Fluctuations and Higher Order Cumulants of the Net Baryon Number, Phys. Rev. C88 (2013) 034911. arXiv:1205.4756, doi: 10.1103/PhysRevC.88.034911.

[260] M. Gazdzicki, M. Gorenstein, M. Mackowiak-Pawlowska, Normalization of strongly intensive quantities, Phys.Rev. C88 (2) (2013) 024907. arXiv:1303.0871, doi:10.1103/ PhysRevC.88.024907.

[261] E. Sangaline, Strongly intensive cumulants: Fluctuation measures for systems with incompletely constrained volumesarXiv: 1505.00261.

[262] M. Bleicher, S. Jeon, V. Koch, Event-by-event fluctuations of the charged particle ratio from nonequilibrium transport theory, Phys.Rev. C62 (2000) 061902. arXiv:hep-ph/ 0006201, doi:10.1103/PhysRevC.62.061902.

[263] M. Sakaida, M. Asakawa, M. Kitazawa, Effects of global charge conservation on time evolution of cumulants of conserved charges in relativistic heavy ion collisions, Phys. Rev. C90 (6) (2014) 064911. arXiv:1409.6866, doi:10.1103/PhysRevC.90.064911.

[264] B. Abelev, et al., Net-Charge Fluctuations in Pb-Pb collisions at $\sqrt{s}_{N N}=2.76 \mathrm{TeV}$, Phys.Rev.Lett. 110 (15) (2013) 152301. arXiv:1207.6068, doi:10.1103/PhysRevLett. 110.152301.

[265] F. Karsch, Determination of Freeze-out Conditions from Lattice QCD Calculations, Central Eur. J. Phys. 10 (2012) 1234-1237. arXiv:1202.4173, doi:10.2478/ s11534-012-0074-3.

[266] A. Bazavov, H. Ding, P. Hegde, O. Kaczmarek, F. Karsch, et al., Freeze-out Conditions in Heavy Ion Collisions from QCD Thermodynamics, Phys.Rev.Lett. 109 (2012) 192302. arXiv:1208.1220, doi:10.1103/PhysRevLett.109.192302.

[267] S. Borsanyi, Z. Fodor, S. Katz, S. Krieg, C. Ratti, et al., Freeze-out parameters: lattice meets experiment, Phys.Rev.Lett. 111 (2013) 062005. arXiv:1305.5161, doi:10.1103/ 
PhysRevLett.111.062005.

[268] S. Borsanyi, Z. Fodor, S. Katz, S. Krieg, C. Ratti, et al., Freeze-out parameters from electric charge and baryon number fluctuations: is there consistency?, Phys.Rev.Lett. 113 (2014) 052301. arXiv:1403.4576, doi:10.1103/PhysRevLett.113.052301.

[269] L. Adamczyk, et al., Energy Dependence of Moments of Net-proton Multiplicity Distributions at RHIC, Phys. Rev. Lett. 112 (2014) 032302. arXiv:1309.5681, doi: 10.1103/PhysRevLett.112.032302.

[270] L. Adamczyk, et al., Beam energy dependence of moments of the net-charge multiplicity distributions in Au+Au collisions at RHIC, Phys. Rev. Lett. 113 (2014) 092301. arXiv: 1402.1558, doi:10.1103/PhysRevLett.113.092301.

[271] P. Braun-Munzinger, A. Kalweit, K. Redlich, J. Stachel, Confronting fluctuations of conserved charges in central nuclear collisions at the LHC with predictions from Lattice QCD, Phys. Lett. B747 (2015) 292-298. arXiv:1412.8614, doi:10.1016/j.physletb.2015.05. 077.

[272] M. A. Stephanov, K. Rajagopal, E. V. Shuryak, Event-by-event fluctuations in heavy ion collisions and the QCD critical point, Phys. Rev. D60 (1999) 114028. arXiv:hep-ph/ 9903292.

[273] B. Berdnikov, K. Rajagopal, Slowing out of equilibrium near the qcd critical point, Phys. Rev. D61 (2000) 105017. arXiv:hep-ph/9912274.

[274] M. Stephanov, Non-Gaussian fluctuations near the QCD critical point, Phys.Rev.Lett. 102 (2009) 032301. arXiv:0809.3450, doi:10.1103/PhysRevLett.102.032301.

[275] V. Skokov, B. Friman, K. Redlich, Quark number fluctuations in the Polyakov loopextended quark-meson model at finite baryon density, Phys.Rev. C83 (2011) 054904. arXiv:1008.4570, doi:10.1103/PhysRevC.83.054904.

[276] M. Stephanov, On the sign of kurtosis near the QCD critical point, Phys.Rev.Lett. 107 (2011) 052301. arXiv:1104.1627, doi:10.1103/PhysRevLett.107.052301.

[277] J. Randrup, Phase transition dynamics for baryon-dense matter, Phys. Rev. C79 (2009) 054911. arXiv:0903.4736, doi:10.1103/PhysRevC.79.054911.

[278] J. Steinheimer, J. Randrup, Spinodal amplification of density fluctuations in fluiddynamical simulations of relativistic nuclear collisions, Phys. Rev. Lett. 109 (2012) 212301. arXiv:1209.2462, doi:10.1103/PhysRevLett.109.212301.

[279] J. Steinheimer, J. Randrup, V. Koch, Non-equilibrium phase transition in relativistic nuclear collisions: Importance of the equation of state, Phys.Rev. C89 (2014) 034901. arXiv: 1311.0999, doi:10.1103/PhysRevC.89.034901.

[280] B. Friman, F. Karsch, K. Redlich, V. Skokov, Fluctuations as probe of the QCD phase transition and freeze-out in heavy ion collisions at LHC and RHIC, Eur.Phys.J. C71 (2011) 1694. arXiv:1103.3511, doi:10.1140/epjc/s10052-011-1694-2.

[281] G. Endrodi, Z. Fodor, S. D. Katz, K. K. Szabo, The QCD phase diagram at nonzero quark density, JHEP 04 (2011) 001. arXiv:1102.1356, doi:10.1007/JHEP04(2011) 001.

[282] O. Kaczmarek, F. Karsch, E. Laermann, C. Miao, S. Mukherjee, et al., Phase boundary for the chiral transition in $(2+1)$-flavor QCD at small values of the chemical potential, Phys.Rev. D83 (2011) 014504. arXiv:1011.3130, doi:10.1103/PhysRevD.83.014504.

[283] J. Gasser, H. Leutwyler, Light Quarks at Low Temperatures, Phys. Lett. B184 (1987) 83. doi: 10.1016/0370-2693(87) 90492-8.

[284] S. Weinberg, Precise relations between the spectra of vector and axial vector mesons, Phys. Rev. Lett. 18 (1967) 507-509. doi:10.1103/PhysRevLett.18.507.

[285] S. Narison, QCD as a Theory of Hadrons: From Partons to Confinement, Cambridge University Press, 2007.

[286] J. I. Kapusta, E. V. Shuryak, Weinberg type sum rules at zero and finite temperature, Phys. Rev. D49 (1994) 4694-4704. arXiv:hep-ph/9312245, doi:10.1103/PhysRevD.49. 4694. 
[287] M. Dey, V. L. Eletsky, B. L. Ioffe, Mixing of vector and axial mesons at finite temperature: an Indication towards chiral symmetry restoration, Phys. Lett. B252 (1990) 620-624. doi : 10.1016/0370-2693(90)90495-R

[288] U. G. Meissner, Low-Energy Hadron Physics from Effective Chiral Lagrangians with Vector Mesons, Phys. Rept. 161 (1988) 213. doi:10.1016/0370-1573(88)90090-7.

[289] M. Bando, T. Kugo, K. Yamawaki, Nonlinear Realization and Hidden Local Symmetries, Phys. Rept. 164 (1988) 217-314. doi:10.1016/0370-1573(88)90019-1.

[290] A. M. Halasz, J. V. Steele, G.-Q. Li, G. E. Brown, Photon rates for heavy ion collisions from hidden local symmetry, Phys. Rev. C58 (1998) 365-375. arXiv:nucl-th/9712006, doi:10.1103/PhysRevC.58.365.

[291] R. Rapp, J. Wambach, H. van Hees, The Chiral Restoration Transition of QCD and Low Mass Dileptons, Landolt-Bornstein 23 (2010) 134. arXiv:0901.3289, doi:10.1007/ 978-3-642-01539-7_6.

[292] W. Peters, M. Post, H. Lenske, S. Leupold, U. Mosel, The Spectral function of the rho meson in nuclear matter, Nucl. Phys. A632 (1998) 109-127. arXiv:nucl-th/9708004, doi:10.1016/S0375-9474(98)00803-3.

[293] G. E. Brown, W. Weise, Pion Scattering and Isobars in Nuclei, Phys. Rept. 22 (1975) 279-337. doi:10.1016/0370-1573(75) 90026-5.

[294] V. Koch, G. Bertsch, Effects of collective potentials on pion spectra in relativistic heavy ion collisions, Nucl.Phys. A552 (1993) 591-604. arXiv:hep-ph/9208232, doi:10.1016/ 0375-9474 (93) 90287-8.

[295] J. Kapusta, Finite Temperature Field Theory, Cambridge University Press, 1989.

[296] E. Braaten, R. D. Pisarski, Calculation of the gluon damping rate in hot QCD, Phys. Rev. D42 (1990) 2156-2160. doi:10.1103/PhysRevD.42.2156.

[297] J.-P. Blaizot, E. Iancu, Ultrasoft amplitudes in hot QCD, Nucl. Phys. B570 (2000) 326358. arXiv:hep-ph/9906485, doi:10.1016/S0550-3213(99)00783-X.

[298] T. Schäfer, K. Schwenzer, Non-Fermi liquid effects in QCD at high density, Phys. Rev. D70 (2004) 054007. arXiv:hep-ph/0405053, doi:10.1103/PhysRevD .70.054007.

[299] D. T. Son, Superconductivity by long range color magnetic interaction in high density quark matter, Phys. Rev. D59 (1999) 094019. arXiv:hep-ph/9812287, doi:10.1103/ PhysRevD.59.094019.

[300] T. Schäfer, Hard loops, soft loops, and high density effective field theory, Nucl. Phys. A728 (2003) 251-271. arXiv:hep-ph/0307074, doi:10.1016/j.nuclphysa.2003.08.028.

[301] R. Casalbuoni, R. Gatto, Effective theory for color flavor locking in high density QCD, Phys. Lett. B464 (1999) 111-116. arXiv: hep-ph/9908227, doi:10.1016/S0370-2693(99) 01032-1.

[302] D. T. Son, M. A. Stephanov, Inverse meson mass ordering in color flavor locking phase of high density QCD, Phys. Rev. D61 (2000) 074012. arXiv:hep-ph/9910491, doi:10. 1103/PhysRevD.61.074012.

[303] A. Kryjevski, T. Schäfer, An Effective theory for baryons in the CFL phase, Phys. Lett. B606 (2005) 52-58. arXiv:hep-ph/0407329, doi:10.1016/j.physletb.2004.11.081.

[304] G. E. Brown, Landau, Brueckner-Bethe, and Migdal Theories of Fermi Systems, Rev. Mod. Phys. 43 (1971) 1-14. doi:10.1103/RevModPhys.43.1.

[305] G. E. Brown, Many Body Problems, North Holland, 1972.

[306] G. Baym, C. Pethick, Landau Fermi Liquid Theory, Wiley, New York, 1991.

[307] G. Baym, S. A. Chin, Landau Theory of Relativistic Fermi Liquids, Nucl. Phys. A262 (1976) 527. doi:10.1016/0375-9474(76)90513-3.

[308] S. Jeon, L. G. Yaffe, From quantum field theory to hydrodynamics: Transport coefficients and effective kinetic theory, Phys. Rev. D53 (1996) 5799-5809. arXiv:hep-ph/9512263, doi:10.1103/PhysRevD.53.5799.

[309] J. P. Blaizot, E. Iancu, A. Rebhan, The Entropy of the QCD plasma, Phys. Rev. Lett. 83 
(1999) 2906-2909. arXiv:hep-ph/9906340, doi:10.1103/PhysRevLett.83.2906.

[310] A. Peshier, Hard gluon damping in hot QCD, Phys. Rev. D70 (2004) 034016. arXiv: hep-ph/0403225, doi:10.1103/PhysRevD.70.034016.

[311] G. Roche, et al., Dielectron Production in Ca + Ca Collisions at 1-a/GeV and 2-a/GeV, Phys. Lett. B226 (1989) 228. doi:10.1016/0370-2693(89)91186-6.

[312] R. J. Porter, et al., Dielectron cross-section measurements in nucleus-nucleus reactions at 1-A/GeV, Phys. Rev. Lett. 79 (1997) 1229-1232. arXiv:nucl-ex/9703001, doi:10.1103/ PhysRevLett.79.1229.

[313] C. Gale, J. I. Kapusta, Dilepton radiation from high temperature nuclear matter, Phys. Rev. C35 (1987) 2107-2116. doi:10.1103/PhysRevC.35.2107.

[314] E. V. Shuryak, Quantum Chromodynamics and the Theory of Superdense Matter, Phys. Rept. 61 (1980) 71-158. doi:10.1016/0370-1573(80)90105-2.

[315] L. D. McLerran, T. Toimela, Photon and Dilepton Emission from the Quark - Gluon Plasma: Some General Considerations, Phys. Rev. D31 (1985) 545. doi:10.1103/ PhysRevD.31.545.

[316] K. Kajantie, M. Kataja, L. D. McLerran, P. V. Ruuskanen, Transverse Flow Effects in Dilepton Emission, Phys. Rev. D34 (1986) 811. doi:10.1103/PhysRevD.34.811.

[317] K. Kajantie, P. V. Ruuskanen, Production of Thermal Dileptons in High-energy Nucleusnucleus Collisions, Z. Phys. C44 (1989) 167. doi:10.1007/BF01548595.

[318] C. Gale, J. I. Kapusta, Vector dominance model at finite temperature, Nucl. Phys. B357 (1991) 65-89. doi:10.1016/0550-3213(91)90459-B.

[319] R. Rapp, J. Wambach, Chiral symmetry restoration and dileptons in relativistic heavy ion collisions, Adv.Nucl.Phys. 25 (2000) 1. arXiv:hep-ph/9909229, doi:10.1007/ 0-306-47101-9_1.

[320] H. van Hees, R. Rapp, Dilepton Radiation at the CERN Super Proton Synchrotron, Nucl. Phys. A806 (2008) 339-387. arXiv:0711.3444, doi:10.1016/j.nuclphysa.2008.03.009.

[321] R. Rapp, G. Chanfray, J. Wambach, Rho meson propagation and dilepton enhancement in hot hadronic matter, Nucl. Phys. A617 (1997) 472-495. arXiv:hep-ph/9702210, doi: 10.1016/S0375-9474(97)00137-1.

[322] R. Arnaldi, et al., First measurement of the rho spectral function in high-energy nuclear collisions, Phys. Rev. Lett. 96 (2006) 162302. arXiv:nucl-ex/0605007, doi:10.1103/ PhysRevLett.96.162302.

[323] R. Arnaldi, et al., NA60 results on thermal dimuons, Eur. Phys. J. C61 (2009) 711-720. arXiv:0812.3053, doi:10.1140/epjc/s10052-009-0878-5.

[324] V. L. Eletsky, M. Belkacem, P. J. Ellis, J. I. Kapusta, Properties of rho and omega mesons at finite temperature and density as inferred from experiment, Phys. Rev. C64 (2001) 035202. arXiv:nucl-th/0104029, doi:10.1103/PhysRevC.64.035202.

[325] R. Arnaldi, et al., Evidence for the production of thermal-like muon pairs with masses above $1 \mathrm{GeV} / c^{2}$ in 158-A-GeV Indium-Indium Collisions, Eur. Phys. J. C59 (2009) 607623. arXiv:0810.3204, doi:10.1140/epjc/s10052-008-0857-2.

[326] J. Ruppert, C. Gale, T. Renk, P. Lichard, J. I. Kapusta, Low mass dimuons produced in relativistic nuclear collisions, Phys. Rev. Lett. 100 (2008) 162301. arXiv:0706.1934, doi:10.1103/PhysRevLett.100.162301.

[327] K. Dusling, I. Zahed, Transverse momentum spectra of dileptons measured by the NA60 Collaboration in In+In collisions at $158 \mathrm{GeV} /$ nucleon, Phys. Rev. C80 (2009) 014902. arXiv:hep-ph/0701253, doi:10.1103/PhysRevC.80.014902.

[328] S. Endres, H. van Hees, J. Weil, M. Bleicher, Coarse-graining approach for dilepton production at energies available at the CERN Super Proton Synchrotron, Phys. Rev. C91 (5) (2015) 054911. arXiv:1412.1965, doi:10.1103/PhysRevC.91.054911.

[329] H. J. Specht, Thermal Dileptons from Hot and Dense Strongly Interacting Matter, AIP Conf. Proc. 1322 (2010) 1-10. arXiv:1011.0615, doi:10.1063/1.3541982. 
[330] G. Agakichiev, et al., Dielectron production in C-12+C-12 collisions at 2-AGeV with HADES, Phys. Rev. Lett. 98 (2007) 052302. arXiv:nucl-ex/0608031, doi:10.1103/ PhysRevLett.98.052302.

[331] G. Agakishiev, et al., Study of dielectron production in $\mathrm{C}+\mathrm{C}$ collisions at 1-A-GeV, Phys. Lett. B663 (2008) 43-48. arXiv:0711.4281, doi:10.1016/j.physletb.2008.03.062.

[332] G. Agakishiev, et al., Origin of the low-mass electron pair excess in light nucleus-nucleus collisions, Phys. Lett. B690 (2010) 118-122. arXiv:0910.5875, doi:10.1016/j.physletb. 2010.05 .010

[333] G. Agakishiev, et al., Dielectron production in $\mathrm{Ar}+\mathrm{KCl}$ collisions at $1.76 \mathrm{~A} \mathrm{GeV}$, Phys. Rev. C84 (2011) 014902. arXiv:1103.0876, doi:10.1103/PhysRevC.84.014902.

[334] L. Adamczyk, et al., Dielectron Mass Spectra from $\mathrm{Au}+\mathrm{Au}$ Collisions at $\sqrt{s_{\mathrm{NN}}}$ $=200 \mathrm{GeV}$, Phys. Rev. Lett. 113 (2) (2014) 022301, [Addendum: Phys. Rev. Lett.113,no.4,049903(2014)]. arXiv:1312.7397, doi:10.1103/PhysRevLett.113.022301, 10.1103/PhysRevLett.113.049903.

[335] L. Adamczyk, et al., Measurements of Dielectron Production in $\mathrm{Au}+\mathrm{Au}$ Collisions at $\sqrt{s_{\mathrm{NN}}}=200 \mathrm{GeV}$ from the STAR Experiment, Phys. Rev. C in pressarXiv:1504.01317.

[336] L. Adamczyk, et al., Energy dependence of acceptance-corrected dielectron excess mass spectrum at mid-rapidity in $\mathrm{Au}+\mathrm{Au}$ collisions at $\sqrt{s_{N N}}=19.6$ and $200 \mathrm{GeV}$, Phys. Lett. B in pressarXiv:1501.05341.

[337] A. Adare, et al., Detailed measurement of the $e^{+} e^{-}$pair continuum in $p+p$ and $\mathrm{Au}+\mathrm{Au}$ collisions at $\sqrt{s_{N N}}=200 \mathrm{GeV}$ and implications for direct photon production, Phys. Rev. C81 (2010) 034911. arXiv:0912.0244, doi:10.1103/PhysRevC.81.034911.

[338] A. Adare, et al., Dielectron production in $\mathrm{Au}+\mathrm{Au}$ collisions at $\sqrt{s_{N N}}=200$ GeVarXiv: 1509.04667

[339] N. Xu, An Overview of STAR Experimental Results, Nucl. Phys. A931 (2014) 1-12. arXiv:1408.3555, doi:10.1016/j.nuclphysa.2014.10.022.

[340] J. Randrup, J. Cleymans, Maximum freeze-out baryon density in nuclear collisions, Phys. Rev. C74 (2006) 047901. arXiv:hep-ph/0607065, doi:10.1103/PhysRevC.74.047901.

[341] D. Adamova, et al., Enhanced production of low mass electron pairs in $40-\mathrm{AGeV} \mathrm{Pb}-\mathrm{Au}$ collisions at the CERN SPS, Phys. Rev. Lett. 91 (2003) 042301. arXiv:nucl-ex/0209024, doi:10.1103/PhysRevLett.91.042301.

[342] S. Endres, H. van Hees, J. Weil, M. Bleicher, Dilepton production and reaction dynamics in heavy-ion collisions at SIS energies from coarse-grained transport simulations, Phys. Rev. C92 (1) (2015) 014911. arXiv:1505.06131, doi:10.1103/PhysRevC.92.014911.

[343] P. M. Hohler, R. Rapp, Is $\rho$-Meson Melting Compatible with Chiral Restoration?, Phys. Lett. B731 (2014) 103-109. arXiv:1311.2921, doi:10.1016/j.physletb.2014.02.021.

[344] R. Rapp, H. van Hees, Thermal Dileptons as Fireball Thermometer and ChronometerarXiv: 1411.4612.

[345] A. Adare, et al., Enhanced production of direct photons in $\mathrm{Au}+\mathrm{Au}$ collisions at $\sqrt{s_{N N}}=$ $200 \mathrm{GeV}$ and implications for the initial temperature, Phys. Rev. Lett. 104 (2010) 132301. arXiv: 0804.4168, doi:10.1103/PhysRevLett.104.132301.

[346] S. Afanasiev, et al., Measurement of Direct Photons in Au+Au Collisions at $\sqrt{s_{N N}}=200$ GeV, Phys. Rev. Lett. 109 (2012) 152302. arXiv:1205.5759, doi:10.1103/PhysRevLett. 109.152302 .

[347] A. Adare, et al., Centrality dependence of low-momentum direct-photon production in $\mathrm{Au}+\mathrm{Au}$ collisions at $\sqrt{s_{N N}}=200 \mathrm{GeV}$, Phys. Rev. C91 (6) (2015) 064904. arXiv: 1405.3940, doi:10.1103/PhysRevC.91.064904.

[348] J. Adam, et al., Direct photon production in Pb-Pb collisions at $\sqrt{s_{N N}}=2.76$ TeVarXiv: 1509.07324.

[349] H. van Hees, C. Gale, R. Rapp, Thermal Photons and Collective Flow at the Relativistic Heavy-Ion Collider, Phys.Rev. C84 (2011) 054906. arXiv:1108.2131, doi: 
10.1103/PhysRevC.84.054906.

[350] L. Adamczyk, et al., Dielectron azimuthal anisotropy at mid-rapidity in Au+Au collisions at $\sqrt{s_{N N}}=200 \mathrm{GeV}$, Phys. Rev. C90 (6) (2014) 064904. arXiv:1402.1791, doi:10. 1103/PhysRevC.90.064904.

[351] P. Braun-Munzinger, K. Redlich, Charmonium production from the secondary collisions at LHC energy, Eur. Phys. J. C 16 (2000) 519-525. arXiv:hep-ph/0001008, doi:10. $1007 / \mathrm{s} 100520000356$.

[352] B. Abelev, et al., Suppression of high transverse momentum D mesons in central Pb$\mathrm{Pb}$ collisions at $\sqrt{s_{\mathrm{NN}}}=2.76 \mathrm{TeV}$, JHEP 1209 (2012) 112. arXiv:1203.2160, doi: 10.1007/JHEP09 (2012) 112 .

[353] A. Adare, et al., Energy Loss and Flow of Heavy Quarks in Au+Au Collisions at $s_{N N}^{1 / 2}$ = 200-GeV, Phys. Rev. Lett. 98 (2007) 172301. arXiv:nucl-ex/0611018, doi:10.1103/ PhysRevLett.98.172301.

[354] B. B. Abelev, et al., Azimuthal anisotropy of $\mathrm{D}$ meson production in $\mathrm{Pb}-\mathrm{Pb}$ collisions at $\sqrt{s_{\mathrm{NN}}}=2.76 \mathrm{TeV}$, Phys. Rev. C90 (3) (2014) 034904. arXiv:1405.2001, doi:10.1103/ PhysRevC.90.034904.

[355] L. Adamczyk, et al., Elliptic flow of non-photonic electrons in Au+Au collisions at $\sqrt{s_{\mathrm{NN}}}=$ 200, 62.4 and 39 GeVarXiv:1405.6348.

[356] M. He, R. J. Fries, R. Rapp, Heavy Flavor at the Large Hadron Collider in a Strong Coupling Approach, Phys. Lett. B735 (2014) 445-450. arXiv:1401.3817, doi:10.1016/ j.physletb.2014.05.050.

[357] T. Matsui, H. Satz, $J / \psi$ Suppression by Quark-Gluon Plasma Formation, Phys. Lett. B178 (1986) 416. doi:10.1016/0370-2693(86)91404-8.

[358] F. Karsch, H. Satz, The Spectral analysis of strongly interacting matter, Z. Phys. C51 (1991) 209-224. doi:10.1007/BF01475790.

[359] S. Digal, P. Petreczky, H. Satz, Quarkonium feed down and sequential suppression, Phys. Rev. D64 (2001) 094015. arXiv:hep-ph/0106017, doi:10.1103/PhysRevD.64.094015.

[360] F. Karsch, D. Kharzeev, H. Satz, Sequential charmonium dissociation, Phys. Lett. B637 (2006) 75-80. arXiv:hep-ph/0512239, doi:10.1016/j.physletb.2006.03.078.

[361] A. Andronic, P. Braun-Munzinger, K. Redlich, J. Stachel, Evidence for charmonium generation at the phase boundary in ultra-relativistic nuclear collisions, Phys. Lett. B 652 (2007) 259-261. arXiv:nucl-th/0701079, doi:10.1016/j.physletb.2007.07.036.

[362] L. Kluberg, H. Satz, Color Deconfinement and Charmonium Production in Nuclear CollisionsarXiv:0901.3831.

[363] A. Mocsy, P. Petreczky, M. Strickland, Quarkonia in the Quark Gluon Plasma, Int. J. Mod. Phys. A28 (2013) 1340012. arXiv:1302.2180, doi:10.1142/S0217751X13400125.

[364] A. Andronic, P. Braun-Munzinger, K. Redlich, J. Stachel, Statistical hadronization of heavy quarks in ultra-relativistic nucleus-nucleus collisions, Nucl. Phys. A789 (2007) 334356. arXiv:nucl-th/0611023, doi:10.1016/j.nuclphysa.2007.02.013.

[365] P. Braun-Munzinger, J. Stachel, The quest for the quark-gluon plasma, Nature 448 (2007) 302-309. doi:10.1038/nature06080.

[366] P. Braun-Munzinger, J. Stachel, Charmonium from Statistical Hadronization of Heavy Quarks: A Probe for Deconfinement in the Quark-Gluon Plasma, Landolt-Bornstein 23 (2010) 424. arXiv:0901.2500, doi:10.1007/978-3-642-01539-7_14.

[367] P. Braun-Munzinger, J. Stachel, On charm production near the phase boundary, Nucl. Phys. A 690 (2001) 119-126. arXiv:nucl-th/0012064, doi:10.1016/S0375-9474(01) 00936-8.

[368] A. Andronic, P. Braun-Munzinger, K. Redlich, J. Stachel, Statistical hadronization of charm in heavy ion collisions at SPS, RHIC and LHC, Phys. Lett. B 571 (2003) 36-44. arXiv:nucl-th/0303036, doi:10.1016/j.physletb.2003.07.066.

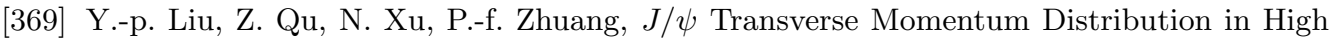


Energy Nuclear Collisions at RHIC, Phys. Lett. B678 (2009) 72-76. arXiv:0901.2757, doi:10.1016/j.physletb.2009.06.006.

[370] X. Zhao, R. Rapp, Medium Modifications and Production of Charmonia at LHC, Nucl. Phys. A859 (2011) 114-125. arXiv:1102.2194, doi:10.1016/j.nuclphysa.2011.05.001.

[371] A. Emerick, X. Zhao, R. Rapp, Bottomonia in the Quark-Gluon Plasma and their Production at RHIC and LHC, Eur. Phys. J. A48 (2012) 72. arXiv:1111.6537, doi: 10.1140/epja/i2012-12072-y.

[372] K. Zhou, N. Xu, Z. Xu, P. Zhuang, Medium effects on charmonium production at ultrarelativistic energies available at the CERN Large Hadron Collider, Phys. Rev. C89 (5) (2014) 054911. arXiv:1401.5845, doi:10.1103/PhysRevC.89.054911.

[373] A. Adare, et al., $J / \psi$ Production vs Centrality, Transverse Momentum, and Rapidity in $\mathrm{Au}+\mathrm{Au}$ Collisions at $\sqrt{s_{N N}}=200 \mathrm{GeV}$, Phys. Rev. Lett. 98 (2007) 232301. arXiv: nucl-ex/0611020, doi:10.1103/PhysRevLett.98.232301.

[374] X. Zhao, A. Emerick, R. Rapp, In-Medium Quarkonia at SPS, RHIC and LHC, Nucl. Phys. A904-905 (2013) 611c-614c. arXiv:1210.6583, doi:10.1016/j.nuclphysa.2013.02.088.

[375] B. Abelev, et al., $J / \psi$ suppression at forward rapidity in $\mathrm{Pb}-\mathrm{Pb}$ collisions at $\sqrt{s_{N N}}=2.76$ TeV, Phys. Rev. Lett. 109 (2012) 072301. arXiv:1202.1383, doi:10.1103/PhysRevLett. 109.072301.

[376] E. Abbas, et al., $J / \psi$ Elliptic Flow in Pb-Pb Collisions at $\sqrt{s_{\mathrm{NN}}}=2.76 \mathrm{TeV}$, Phys. Rev. Lett. 111 (2013) 162301. arXiv:1303.5880, doi:10.1103/PhysRevLett.111.162301.

[377] L. Adamczyk, et al., Measurement of $J / \psi$ Azimuthal Anisotropy in $\mathrm{Au}+\mathrm{Au}$ Collisions at $\sqrt{s_{N N}}=200 \mathrm{GeV}$, Phys. Rev. Lett. 111 (5) (2013) 052301. arXiv:1212.3304, doi: 10.1103/PhysRevLett.111.052301.

[378] F. Prino, et al., $J / \psi$ azimuthal anisotropy relative to the reaction plane in $\mathrm{Pb}-\mathrm{Pb}$ collisions at $158 \mathrm{GeV}$ per nucleon, Eur. Phys. J. C61 (2009) 853-858. arXiv:0906.5376, doi: 10.1140/epjc/s10052-009-0907-4.

[379] S. Chatrchyan, et al., Suppression of non-prompt $J / \psi$, prompt $J / \psi$, and $\Upsilon(1 S)$ in $\mathrm{PbPb}$ collisions at $\sqrt{s_{N N}}=2.76 \mathrm{TeV}$, JHEP 05 (2012) 063. arXiv:1201.5069, doi:10.1007/ JHEP05 (2012) 063

[380] H. Liu, K. Rajagopal, U. A. Wiedemann, An AdS/CFT Calculation of Screening in a Hot Wind, Phys. Rev. Lett. 98 (2007) 182301. arXiv:hep-ph/0607062, doi:10.1103/ PhysRevLett.98.182301.

[381] A. Mocsy, P. Petreczky, Can quarkonia survive deconfinement?, Phys. Rev. D77 (2008) 014501. arXiv:0705.2559, doi:10.1103/PhysRevD.77.014501.

[382] M. Laine, News on hadrons in a hot medium, in: Proceedings, 14th International Conference on Hadron spectroscopy (Hadron 2011), 2011. arXiv:1108.5965.

[383] A. Adare, et al., Nuclear Modification of $\psi^{\prime}, \chi_{c}$, and $J / \psi$ Production in $\mathrm{d}+\mathrm{Au}$ Collisions at $\sqrt{s_{N N}}=200 \mathrm{GeV}$, Phys. Rev. Lett. 111 (20) (2013) 202301. arXiv:1305.5516, doi: 10.1103/PhysRevLett.111.202301.

[384] B. B. Abelev, et al., Suppression of $\psi(2 \mathrm{~S})$ production in $\mathrm{p}-\mathrm{Pb}$ collisions at $\sqrt{s_{N N}}=5.02$ TeV, JHEP 12 (2014) 073. arXiv:1405.3796, doi:10.1007/JHEP12(2014) 073.

[385] S. Chatrchyan, et al., Observation of sequential Upsilon suppression in $\mathrm{PbPb}$ collisions, Phys. Rev. Lett. 109 (2012) 222301. arXiv:1208.2826, doi:10.1103/PhysRevLett.109. 222301.

[386] S. Chatrchyan, et al., Event activity dependence of $\mathrm{Y}(\mathrm{nS})$ production in $\sqrt{s_{N N}}=5.02$ $\mathrm{TeV} \mathrm{pPb}$ and $\sqrt{s}=2.76 \mathrm{TeV}$ pp collisions, JHEP 04 (2014) 103. arXiv:1312.6300, doi: 10.1007/JHEP04 (2014) 103 .

[387] B. B. Abelev, et al., Suppression of $\Upsilon(1 S)$ at forward rapidity in $\mathrm{Pb}-\mathrm{Pb}$ collisions at $\sqrt{s_{\mathrm{NN}}}=2.76 \mathrm{TeV}$, Phys. Lett. B738 (2014) 361-372. arXiv:1405.4493, doi:10.1016/j . physletb.2014.10.001.

[388] A. Adare, et al., Measurement of $\Upsilon(1 S+2 S+3 S)$ production in $p+p$ and $\mathrm{Au}+\mathrm{Au}$ 
collisions at $\sqrt{s_{N N}}=200 \mathrm{GeV}$, Phys. Rev. C91 (2) (2015) 024913. arXiv:1404.2246, doi:10.1103/PhysRevC.91.024913.

[389] A. Andronic, et al., Heavy-flavour and quarkonium production in the LHC era: from proton-proton to heavy-ion collisionsarXiv:1506.03981.

[390] A. Andronic, Experimental results and phenomenology of quarkonium production in relativistic nuclear collisions, Nucl. Phys. A931 (2014) 135-144. arXiv:1409.5778, doi:10.1016/j.nuclphysa.2014.10.009. 